\title{
2010
}

Brookhaven National Laboratory Annual Illness and Injury Surveillance Report

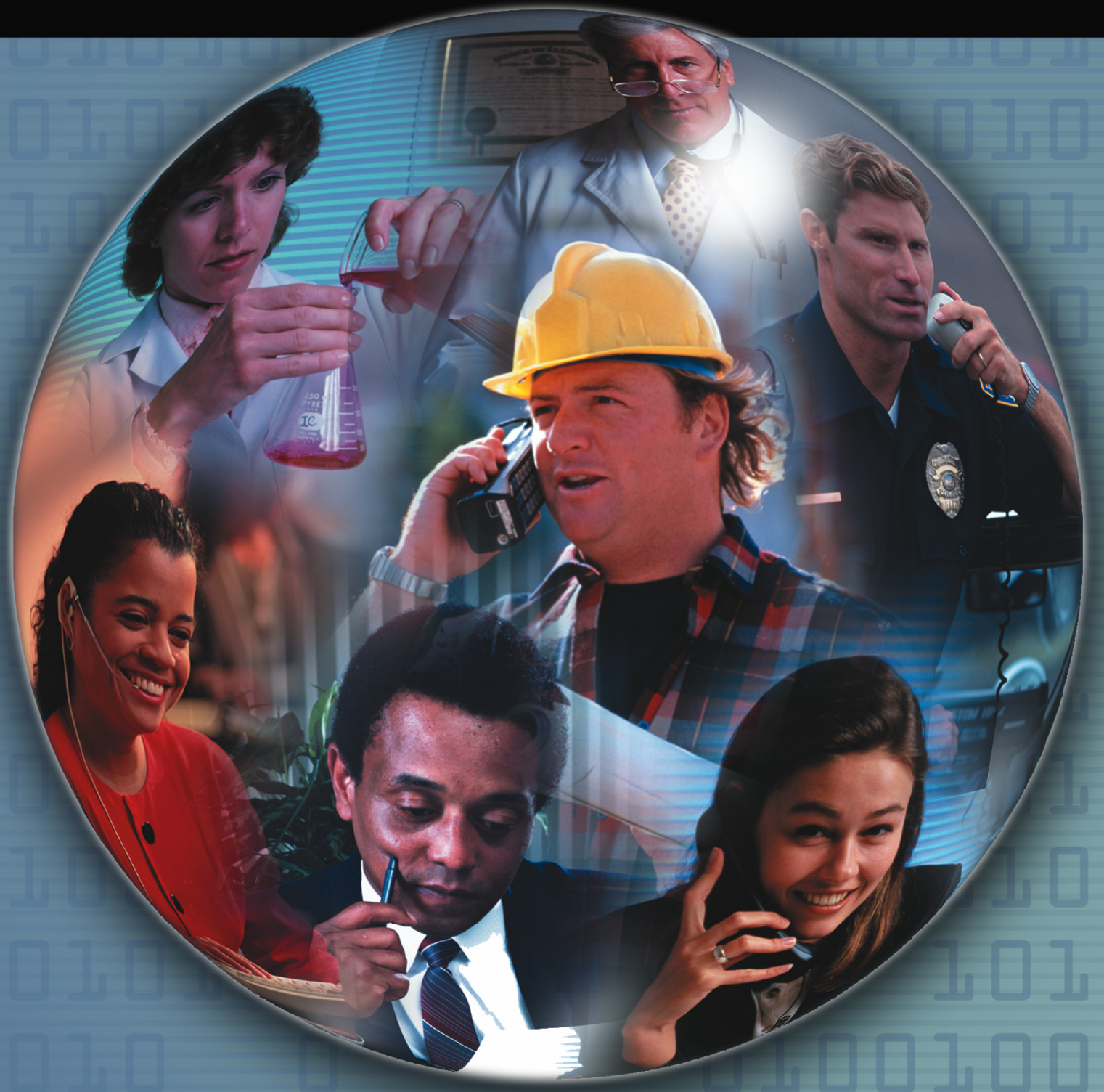




\section{Brookhaven National Laboratory 2010 Illness and Injury Surveillance Report}

Questions or comments about this report or the Illness and Injury Surveillance Program (IISP) may be directed to:

E-mail:

Dr. Cliff Strader at cliff.strader@hq.doe.gov or Dr. Bonnie Richter at bonnie.richter@hq.doe.gov

or direct letters to:

Mail Stop HS-13 / GTN Building

U.S. Department of Energy

1000 Independence Avenue, S.W.

Washington, DC 20585-0270

Additional information about the Department of Energy's Office of Illness and Injury Prevention Programs, the IISP, and annual reports for DOE sites participating in this program can be found at:

http://www.hss.energy.gov/healthsafety/WSHP/epi/surv/

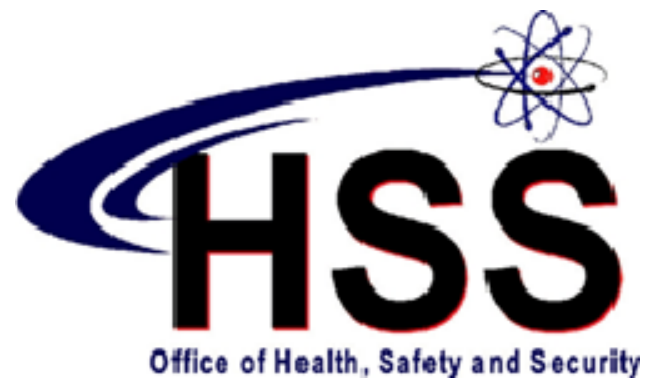

ACKNOWLEDGEMENT

LifeART images copyright 2000 Lippincott Williams \& Wilkins. All rights reserved.

This document was produced under contract number DE-AC05-06OR23100 between the

U.S. Department of Energy and Oak Ridge Associated Universities. 


\section{Brookhaven National Laboratory \\ 2010 Illness and Injury Surveillance Report}

At A Glance

NOTE: In previous years, the number of days absent or lost and the age of the work force were reported as averages. Beginning in 2010, they are being reported as medians. A median is the value in a population that half the values exceed and half fall short of it. The median is less affected than the average when the distribution contains a few very large or very small values and provides a more representative summary measure.

The BNL work force totaled 3,166 workers in 2010, a slight increase from 3,031 workers in 2009. The work force has increased 14 percent from 2006 to 2010.

The median age of the work force at BNL has changed little since 2006. Both men and women had a median age of 48 years in 2006, compared with a median age of 49 years in 2010 .

There were a total of 169 absences among 119 women, a rate of 20 absences per 100 workers $(169 / 835)$. Among 247 men, there were 320 absences, a rate of 14 absences per 100 workers $(320 / 2,331)$. The top three diagnostic categories reported by women were respiratory and musculoskeletal conditions and unspecified symptoms. Thirty-six percent of the unspecified symptoms were digestive symptoms, 13 percent were due to headache, and 23 percent were all other symptoms. Among men, the top three diagnostic categories were musculoskeletal and respiratory conditions and injuries.

The injury rate among women increased 61 precent from 2009 to 2010 and was 3 times higher in 2010 than it was in 2006. The injury rate decreased 9 percent among men from 2009 to 2010, but it has doubled in the period from 2006 to 2010. The changes in injury rates over the period for men and women were not due to any particular type of injury. The rate of musculoskeletal conditions among women increased 29 percent from 2009 to 2010 due to joint disorders after declining steadily from 2007 to 2009. The rate of 
musculoskeletal conditions decreased for men for the second year due to a reduction in diagnoses of rheumatism.

Service workers had the highest rate for all diagnoses combined among women in 2010 (1157 diagnoses per 1,000 workers), an increase of 3 percent from the rate in 2009. Crafts workers had the highest rate among men (654 diagnoses per 1,000 workers), a 9 percent decrease from the rate in 2009. Service workers have had the highest diagnosis rate among women every year since 2006 . This is the third time since 2006 that Craft workers have had the highest rate among men.

BNL reported 53 definite and 11 possible occupational sentinel health event (SHEO) diagnoses in 2010. Injuries (23 diagnoses) and musculoskeletal conditions (18 diagnoses) accounted for most of the SHEO diagnoses. There were 13 carpal tunnel syndrome diagnoses among 11 people, 8 of whom were 50 to 59 years of age. The 53 definite SHEOs were reported by 36 workers. Three-quarters of the workers were in the Professional, Service, and Crafts job categories, while the remaining were in 3 other job categories.

Twenty-nine OSHA-recordable events were reported at BNL in 2010, a 28 percent decrease from 2009. Accidents were the cause of 18 of these events. The most common type of accident was falls, which accounted for 39 percent of the accidents; overexertion and strenuous movements were the second most common accident (33 percent). Twenty-seven of the 29 diagnoses reported for OSHA events (93 percent) were injuries, mainly unspecified injuries of the upper limb.

The rate of OSHA events was 1 event per 100 workers in 2010. Crafts and Security and Fire workers had the highest rates of OSHA events among men in 2010 (6.2 events per 100 workers). Crafts and Security and Fire workers have had the highest rates of OSHA events since 2006. Among women, the 5 OSHA events were reported by Professional and Administrative Support workers. Lost and restricted workdays decreased 67 percent from 1,519 days in 2009 to a 5year low of 505 days in 2010, after steadily increasing each year from 2006 to 2009. 


\section{The Brookhaven National Laboratory Work Force - 2010}

The Work Force by Gender and Age ............ 1

The Work Force by Gender and Job

Category 1

\section{Number and Length of Absences}

Absence Rate by Gender and Age 2

Number of Days Absent by

Gender and Age

Absence Rate by Job Category

and Gender 3

Median Duration of Absence by

Job Category and Gender. ... 3

\section{Diagnostic Categories}

Number of Diagnoses and Lost Calendar Days by Diagnostic Category

(Categorized by ICD-9-CM) and Gender . .4

Common Diagnoses Among Female

Workers in 2010

Common Diagnoses Among Male

Workers in 2010 .. 6

Number of Most Frequently Reported

Diagnoses by Job Category and Gender 7

\section{Rates of Disease Occurrence}

Rates for All Illnesses and Injuries Combined by Job Category, Gender, and Age

Rates for Selected Diagnostic Categories by Job Category, Gender, and Age .8

\section{Time Trends}

Age-Adjusted Rates for All Diagnoses Combined Among Women and Men from 2006 to 2010
Age-Adjusted Rates for Selected Diagnostic Categories Among Women and Men from 2006 to 2010

Age-Adjusted Rates for All Diagnoses

Combined Among Women and Men by Job

Category from 2006 to $2010 \ldots \ldots \ldots \ldots \ldots \ldots \ldots \ldots . \ldots 12$

\section{Sentinel Health Events for Occupations (SHEOs)}

Characteristics of SHEOs by Gender 13

SHEO Diagnoses by Gender 13

Occupational Safety and Health Administration (OSHA)-Recordable Events

OSHA-Recordable Events by Gender and Age. 14

OSHA-Recordable Events by Job

Category and Gender 14

\section{Diagnostic and Accident Categories for OSHA-Recordable Events}

OSHA-Recordable Diagnoses by

Diagnostic Category and Gender

OSHA-Recordable Accidents by Type

and Gender

Rates of OSHA-Recordable Events

OSHA-Recordable Rates by Age and Job

Categories Among Women, All Diagnoses

Combined

OSHA-Recordable Rates by Age and Job

Categories Among Men, All Diagnoses

Combined

Time Trends for OSHA-Recordable Events

Age-Adjusted Rates for All OSHA-Recordable Diagnoses Combined Among Women and Men by Job Category from 2006 to 2010 .

\section{Appendices}


The Brookhaven National Laboratory Work Force - 2010

Figure 1. The Work Force by Gender and Age

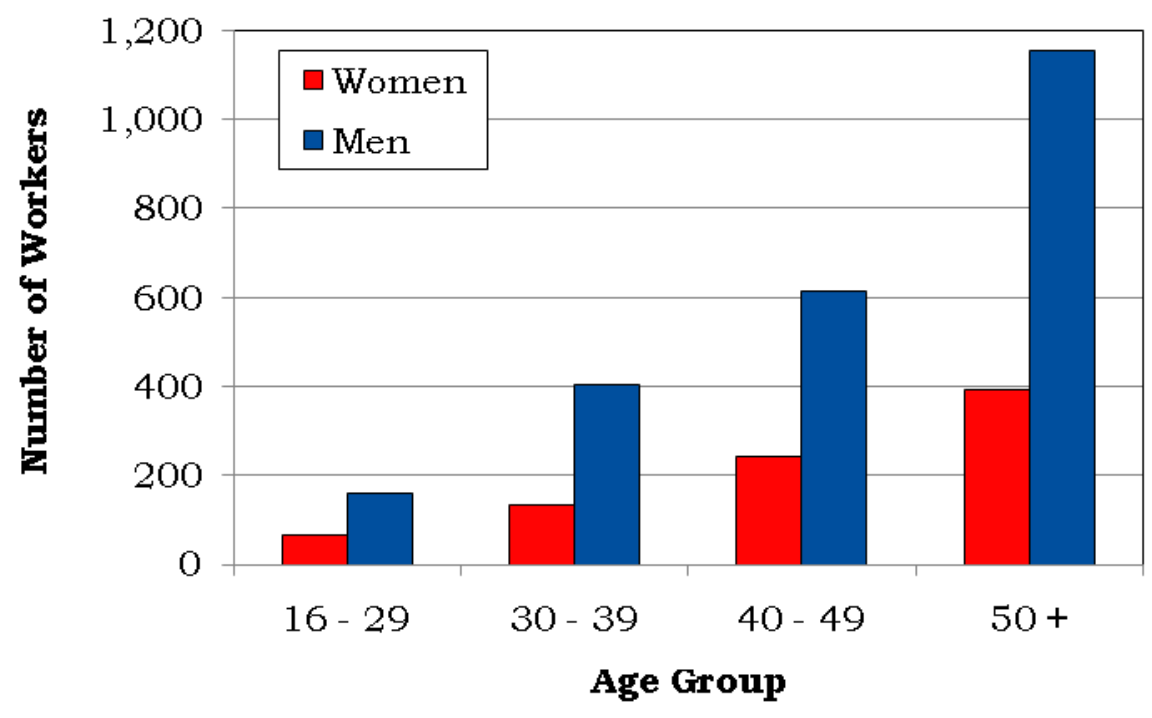

Figure 2. The Work Force by Gender and Job Category

\begin{tabular}{|l|c|c|}
\hline \multicolumn{1}{|c|}{ Job Category } & Women & M en \\
\hline \multirow{2}{*}{ Professional } & 418 & 1,487 \\
& $50 \%$ & $64 \%$ \\
\hline \multirow{2}{*}{ Administrative Support } & 321 & 46 \\
& $38 \%$ & $2 \%$ \\
\hline \multirow{2}{*}{ Technical Support } & 41 & 397 \\
& $5 \%$ & $17 \%$ \\
\hline \multirow{2}{*}{ Service } & 46 & 190 \\
& $5 \%$ & $8 \%$ \\
\hline \multirow{2}{*}{ Security and Fire } & 6 & 65 \\
& $1 \%$ & $3 \%$ \\
\hline \multirow{2}{*}{ Crafts } & 3 & 146 \\
& $<1 \%$ & $6 \%$ \\
\hline \multirow{2}{*}{ Total } & 0 & 0 \\
& $0 \%$ & $0 \%$ \\
\hline
\end{tabular}




\section{Number and Length of Absences}

Figure 3. Absence Rate by Gender and Age

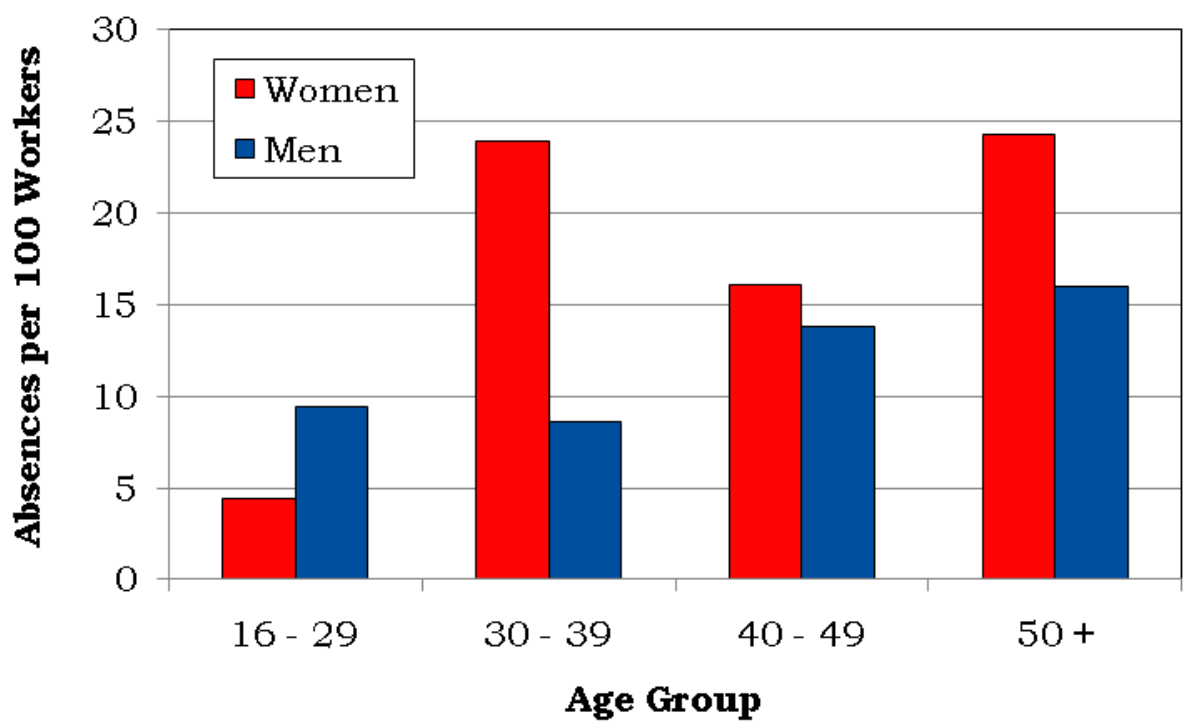

Figure 4. Number of Days Absent by Gender and Age

\begin{tabular}{|c|c|c|c|c|}
\hline \multirow{3}{*}{ Gender } & \multirow{2}{*}{ Age } & \multirow{2}{*}{$\begin{array}{c}\text { Number of } \\
\text { Absences }\end{array}$} & \multicolumn{2}{|c|}{ Number of Days Absent } \\
\cline { 3 - 5 } & & Total & M edian \\
\hline \multirow{4}{*}{ Women* } & $16-29$ & 3 & 9 & 2 \\
\cline { 2 - 5 } & $30-39$ & 32 & 852 & 7 \\
\cline { 2 - 5 } & $40-49$ & 39 & 975 & 7 \\
\cline { 2 - 5 } & $50+$ & 95 & 2,251 & 7 \\
\cline { 2 - 5 } & Total & 169 & 4,087 & 7 \\
\hline \multirow{4}{*}{ Men } & $16-29$ & 15 & 92 & 5 \\
\cline { 2 - 5 } & $30-39$ & 35 & 448 & 7 \\
\cline { 2 - 5 } & $40-49$ & 85 & 1,286 & 10 \\
\cline { 2 - 5 } & $50+$ & 185 & 5,175 & 8 \\
\cline { 2 - 5 } & Total & 320 & 7,001 & 7 \\
\hline
\end{tabular}

*Normal pregnancies were excluded from absences for women. 
Figure 5. Absence Rate by Job Category and Gender

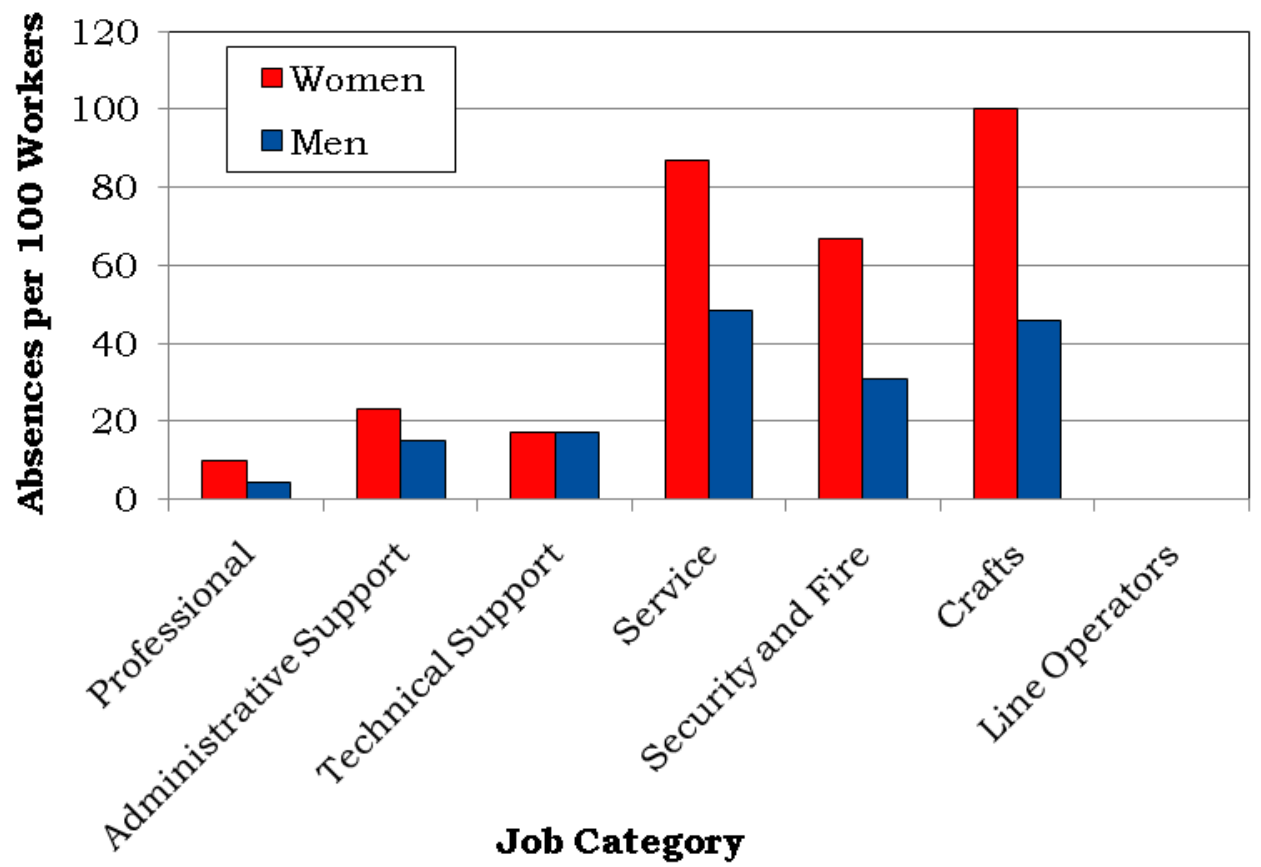

Figure 6. Median Duration of Absence by Job Category and Gender

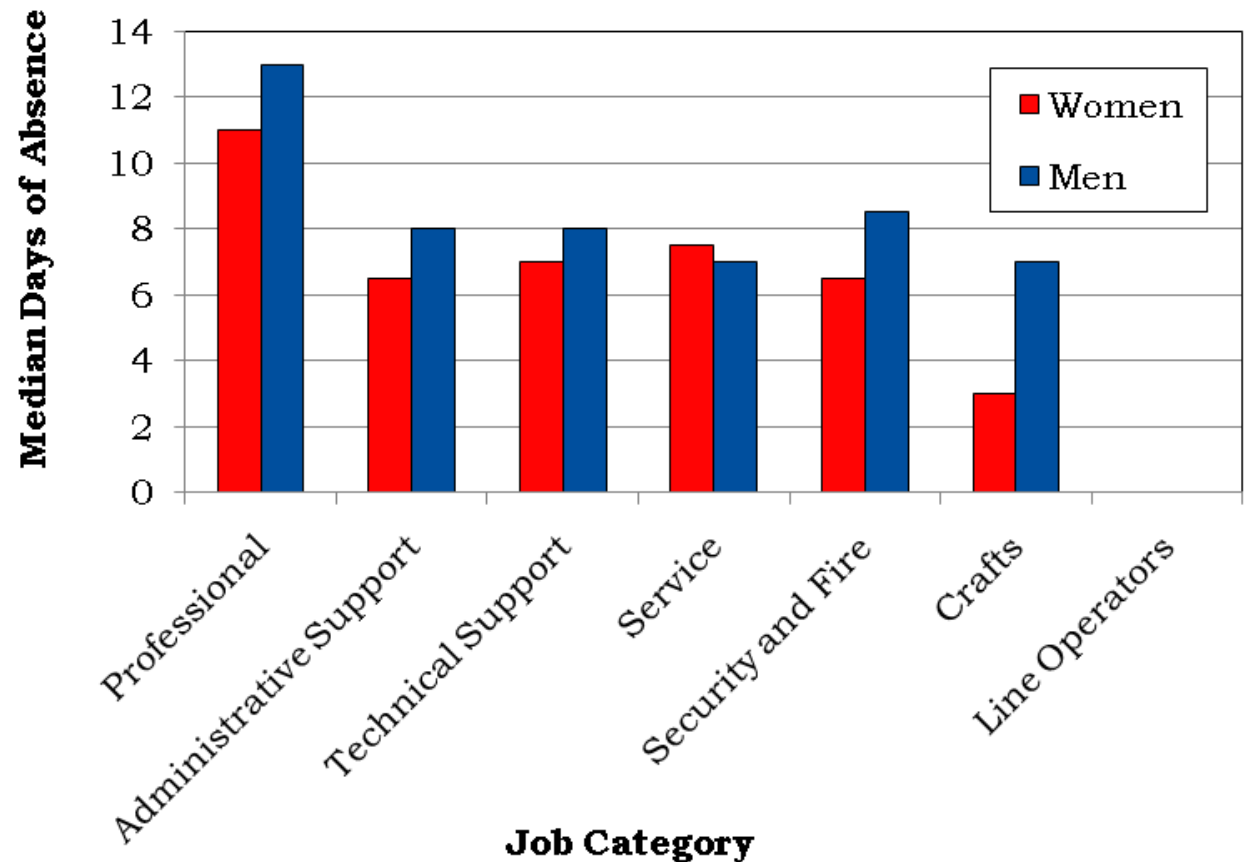




\section{Diagnostic Categories}

Figure 7. Number of Diagnoses and Lost Calendar Days by Diagnostic Category (Categorized by ICD-9-CM) and Gender

\begin{tabular}{|l|c|c|c|c|}
\hline \multirow{2}{*}{ Diagnostic Category } & \multicolumn{2}{c|}{ Women } & \multicolumn{2}{c|}{ M en } \\
\cline { 2 - 5 } & Number of & $\begin{array}{c}\text { Number } \\
\text { of Lost } \\
\text { Calendar } \\
\text { Days }\end{array}$ & $\begin{array}{c}\text { Number of } \\
\text { Diagnoses }\end{array}$ & $\begin{array}{c}\text { Number } \\
\text { of Lost } \\
\text { Calendar } \\
\text { Days }\end{array}$ \\
\hline Benign Growths & $\mathbf{5}$ & $\mathbf{1 8 4}$ & $\mathbf{1}$ & $\mathbf{6}$ \\
\hline Blood & $\mathbf{0}$ & $\mathbf{0}$ & $\mathbf{0}$ & $\mathbf{0}$ \\
\hline Cancer & $\mathbf{4}$ & $\mathbf{4 1}$ & $\mathbf{1 8}$ & $\mathbf{6 0 6}$ \\
\hline Digestive & $\mathbf{1 3}$ & 291 & $\mathbf{5 2}$ & $\mathbf{8 5 2}$ \\
\hline Endocrine/Metabolic & $\mathbf{3}$ & $\mathbf{5 5 7}$ & $\mathbf{1 2}$ & $\mathbf{2 9 8}$ \\
\hline Existing Birth Condition & $\mathbf{1}$ & $\mathbf{5 3}$ & $\mathbf{0}$ & $\mathbf{0}$ \\
\hline Genitourinary & $\mathbf{1 3}$ & $\mathbf{1 2 7}$ & $\mathbf{9}$ & $\mathbf{5 5}$ \\
\hline Heart/Circulatory & $\mathbf{7}$ & $\mathbf{5 7 5}$ & $\mathbf{2 9}$ & $\mathbf{7 6 4}$ \\
\hline Infections/Parasites & $\mathbf{6}$ & $\mathbf{4 2}$ & $\mathbf{1 6}$ & $\mathbf{1 2 3}$ \\
\hline Injury & $\mathbf{2 8}$ & $\mathbf{1 , 0 9 2}$ & $\mathbf{6 7}$ & $\mathbf{1 , 8 1 6}$ \\
\hline Miscarriage & $\mathbf{0}$ & $\mathbf{0}$ & $\mathbf{N A}$ & NA \\
\hline Musculoskeletal & $\mathbf{5 2}$ & $\mathbf{1 , 9 8 6}$ & $\mathbf{8 0}$ & $\mathbf{1 , 8 5 1}$ \\
\hline Nervous System & $\mathbf{1 7}$ & $\mathbf{3 8 8}$ & $\mathbf{2 7}$ & $\mathbf{8 4 0}$ \\
\hline Psychological & $\mathbf{1 0}$ & $\mathbf{2 9 8}$ & $\mathbf{7}$ & $\mathbf{2 0 3}$ \\
\hline Respiratory & $\mathbf{5 6}$ & $\mathbf{3 5 6}$ & $\mathbf{5 5}$ & $\mathbf{6 3 2}$ \\
\hline Skin & $\mathbf{3 0}$ & $\mathbf{2 2}$ & $\mathbf{7}$ & $\mathbf{1 2 2}$ \\
\hline Unspecified Symptoms & & $\mathbf{3 3}$ & $\mathbf{3 4 4}$ \\
\hline
\end{tabular}

Note: Lost calendar days for each absence are counted more than once when multiple diagnoses occur in different diagnostic categories for the same absence. 


\section{Figure 8. Common Diagnoses Among Female Workers in 2010}

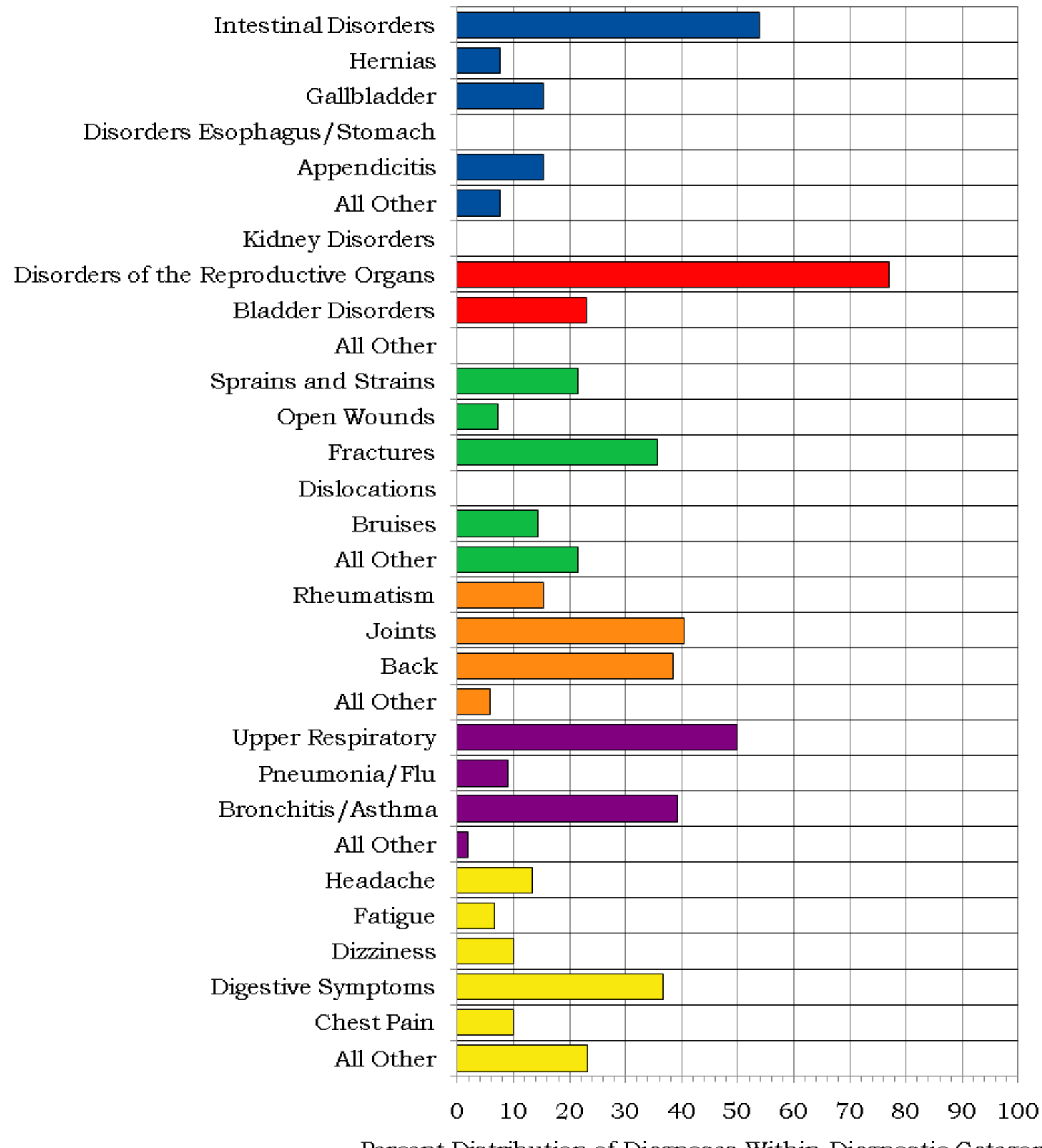

Percent Distribution of Diagnoses Within Diagnostic Category

Digestive, 13 Diagnoses

Genitourinary, 13 Diagnoses

Injury, 28 Diagnoses
Musculoskeletal, 52 Diagnoses

Respiratory, 56 Diagnoses

Unspecified Symptoms, 30 Diagnoses 


\section{Figure 9. Common Diagnoses Among Male Workers in 2010}

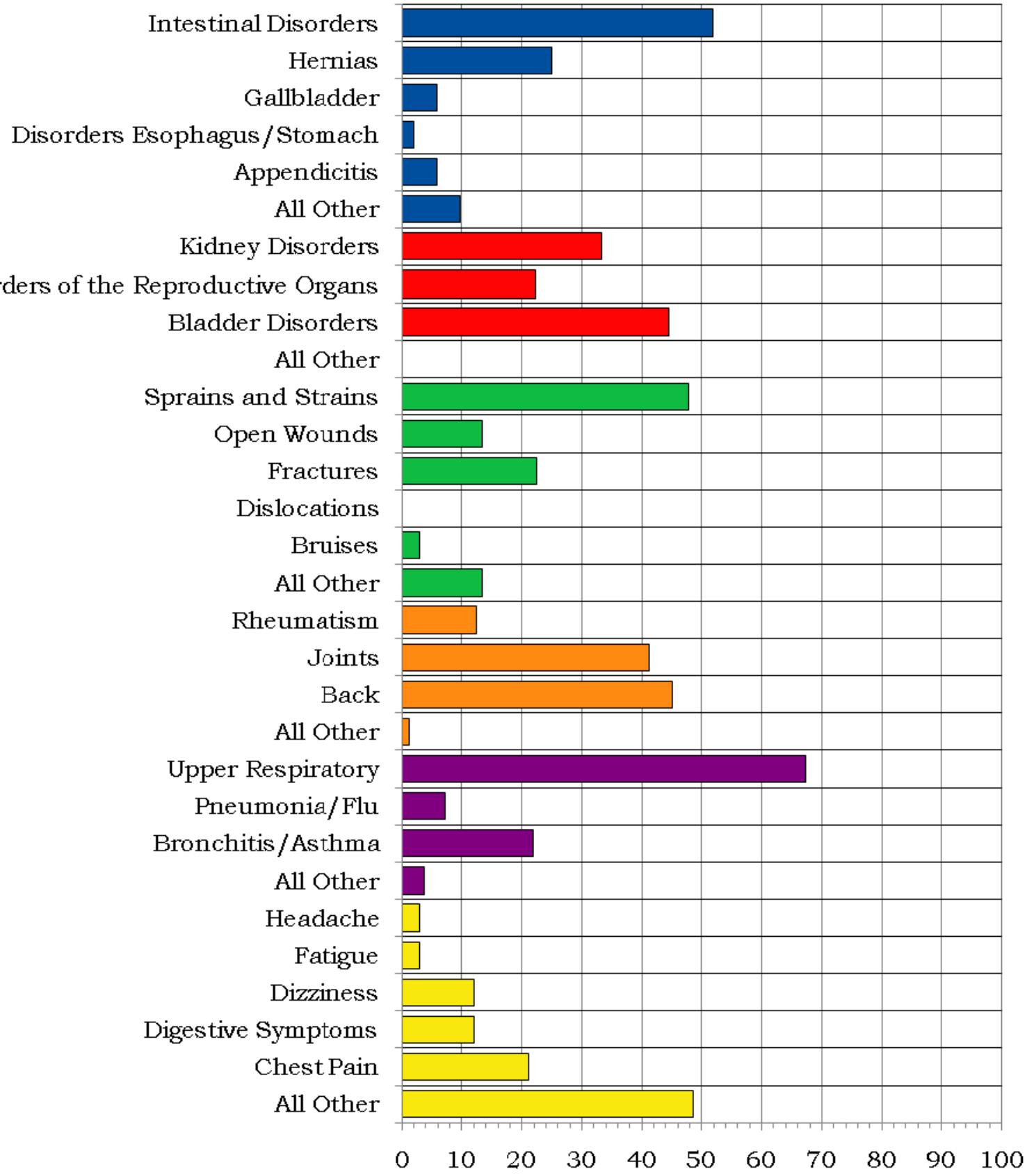

Percent Distribution of Diagnoses Within Diagnostic Category

Digestive, 52 Diagnoses

Genitourinary, 9 Diagnoses

Injury, 67 Diagnoses
Musculoskeletal, 80 Diagnoses

Respiratory, 55 Diagnoses

Unspecified Symptoms, 33 Diagnoses 


\section{Figure 10. Number of Most Frequently Reported Diagnoses by Job Category and Gender}

\begin{tabular}{|c|c|c|c|c|}
\hline Job Category & Men & & Women & \\
\hline \multirow{4}{*}{ Professional } & Digestive & 15 & Injury & 17 \\
\hline & M usculoskeletal & 13 & M usculoskeletal & 9 \\
\hline & Injury & 11 & Respiratory & 8 \\
\hline & & & Unspecified Symptoms & 8 \\
\hline \multirow{5}{*}{ Administrative Support } & Respiratory & 3 & M usculoskeletal & 23 \\
\hline & Digestive & 2 & Respiratory & 22 \\
\hline & Injury & 1 & Unspecified Symptoms & 18 \\
\hline & Heart/Circulatory & 1 & & \\
\hline & skin & 1 & & \\
\hline \multirow{6}{*}{ Technical Support } & Injury & 16 & Respiratory & 2 \\
\hline & M usculoskeletal & 16 & Injury & 2 \\
\hline & Respiratory & 14 & M usculoskeletal & 1 \\
\hline & & & Digestive & 1 \\
\hline & & & Benign Growths & 1 \\
\hline & & & Genitourinary & 1 \\
\hline \multirow{3}{*}{ Service } & M usculoskeletal & 29 & Respiratory & 18 \\
\hline & Injury & 17 & M usculoskeletal & 17 \\
\hline & Digestive & 15 & Injury & 5 \\
\hline \multirow{3}{*}{ Security and Fire } & M usculoskeletal & 6 & Respiratory & 5 \\
\hline & Injury & 3 & Nervous System & 1 \\
\hline & Heart/Circulatory & 3 & Infections/Parasites & 1 \\
\hline \multirow{3}{*}{ Crafts } & Injury & 19 & M usculoskeletal & 2 \\
\hline & M usculoskeletal & 16 & Respiratory & 1 \\
\hline & Respiratory & 14 & Nervous System & 1 \\
\hline Line Operators & & 0 & & 0 \\
\hline
\end{tabular}




\section{Rates of Disease Occurrence}

Figure 11. Rates for All Illnesses and Injuries Combined by Job Category, Gender, and Age

\begin{tabular}{|c|c|c|c|c|}
\hline \multirow{2}{*}{$\begin{array}{c}\text { All Illnesses \& } \\
\text { Injuries Combined }\end{array}$} & \multicolumn{4}{|c|}{ Rate per 1,000} \\
\hline & Job Category & Age & Men & Women \\
\hline & \multirow{2}{*}{ Professional } & $<50$ & 51 & 63 \\
\hline & & $50+$ & 63 & 291 \\
\hline & \multirow{2}{*}{ Administrative Support } & $<50$ & 71 & 435 \\
\hline & & $50+$ & 219 & 262 \\
\hline & \multirow{2}{*}{ Technical Support } & $<50$ & 170 & 316 \\
\hline & & $50+$ & 293 & 91 \\
\hline & \multirow{2}{*}{ Service } & $<50$ & 578 & 1,111 \\
\hline & & $50+$ & 660 & 1,263 \\
\hline & \multirow{2}{*}{ Security and Fire } & $<50$ & 324 & 750 \\
\hline & & $50+$ & 393 & 2,000 \\
\hline & \multirow{2}{*}{ Crafts } & $<50$ & 474 & 500 \\
\hline & & $50+$ & 632 & 3,000 \\
\hline & \multirow{2}{*}{ Line Operators } & $<50$ & 0 & 0 \\
\hline & & $50+$ & $\mathbf{0}$ & 0 \\
\hline
\end{tabular}

Figure 12. Rates for Selected Diagnostic Categories by Job Category, Gender, and Age

\begin{tabular}{|c|c|c|c|c|}
\hline \multirow{2}{*}{ Cancer } & \multicolumn{4}{|c|}{ Rate per 1,000} \\
\hline & Job Category & Age & Men & Women \\
\hline & Professional & $<50$ & 0 & 0 \\
\hline & 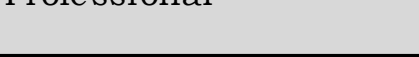 & $50+$ & 9 & 6 \\
\hline & Administrative Support & $<50$ & 0 & 0 \\
\hline & & $50+$ & 0 & 5 \\
\hline & Technical Support & $<50$ & 6 & 0 \\
\hline & & $50+$ & 9 & 0 \\
\hline & Service & $<50$ & 0 & 0 \\
\hline & Nelvice & $50+$ & 30 & 0 \\
\hline & Security and Fire & $<50$ & 27 & 0 \\
\hline & Secuity and rile & $50+$ & 36 & 0 \\
\hline & Crafts & $<50$ & 0 & 0 \\
\hline & & $50+$ & 0 & 0 \\
\hline & & $<50$ & 0 & 0 \\
\hline & 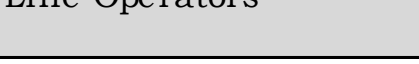 & $50+$ & 0 & 0 \\
\hline
\end{tabular}


Figure 12. Rates for Selected Diagnostic Categories by Job Category, Gender, and Age (Continued)

\begin{tabular}{|l|l|c|c|c|}
\hline \multirow{2}{*}{ Heart/Circulatory } & \multicolumn{4}{|c|}{ Rate per 1,000 } \\
\cline { 2 - 5 } & \multicolumn{1}{|c|}{ Job Category } & Age & M en & W omen \\
\hline \multirow{2}{*}{ Professional } & $<50$ & $\mathbf{1}$ & $\mathbf{0}$ \\
\cline { 2 - 5 } & $50+$ & $\mathbf{6}$ & $\mathbf{6}$ \\
\hline \multirow{2}{*}{ Administrative Support } & $<50$ & $\mathbf{0}$ & $\mathbf{1 4}$ \\
\cline { 2 - 5 } & $50+$ & $\mathbf{3 1}$ & $\mathbf{1 6}$ \\
\hline \multirow{2}{*}{ Technical Support } & $<50$ & $\mathbf{6}$ & $\mathbf{0}$ \\
\cline { 2 - 5 } & & $50+$ & $\mathbf{3 9}$ & $\mathbf{0}$ \\
\hline \multirow{2}{*}{ Service } & $<50$ & $\mathbf{0}$ & $\mathbf{3 7}$ \\
\cline { 2 - 5 } & $50+$ & $\mathbf{9 0}$ & $\mathbf{0}$ \\
\cline { 2 - 5 } & \multirow{2}{*}{ Security and Fire } & $<50$ & $\mathbf{0}$ & $\mathbf{0}$ \\
\cline { 2 - 5 } & $50+$ & $\mathbf{1 0 7}$ & $\mathbf{0}$ \\
\cline { 2 - 5 } & \multirow{2}{*}{ Crafts } & $<50$ & $\mathbf{0}$ & $\mathbf{0}$ \\
\cline { 2 - 5 } & $50+$ & $\mathbf{1 5}$ & $\mathbf{0}$ \\
\cline { 2 - 5 } & \multirow{2}{*}{ Line Operators } & $<50$ & $\mathbf{0}$ & $\mathbf{0}$ \\
\cline { 2 - 5 } & $50+$ & $\mathbf{0}$ & $\mathbf{0}$ \\
\hline
\end{tabular}

\begin{tabular}{|c|c|c|c|c|}
\hline \multirow{2}{*}{ Respiratory } & \multicolumn{4}{|c|}{ Rate per 1,000} \\
\hline & Job Category & Age & Men & Women \\
\hline & \multirow{2}{*}{ Professional } & $<50$ & 8 & 0 \\
\hline & & $50+$ & 6 & 48 \\
\hline & \multirow{2}{*}{ Administrative Support } & $<50$ & 0 & 80 \\
\hline & & $50+$ & 94 & 60 \\
\hline & \multirow{2}{*}{ Technical Support } & $<50$ & 30 & 53 \\
\hline & & $50+$ & 39 & 45 \\
\hline & \multirow{2}{*}{ Service } & $<50$ & 89 & 370 \\
\hline & & $50+$ & 40 & 421 \\
\hline & \multirow{2}{*}{ Security and Fire } & $<50$ & 27 & 500 \\
\hline & & $50+$ & 36 & 1,500 \\
\hline & \multirow{2}{*}{ Crafts } & $<50$ & 77 & 500 \\
\hline & & $50+$ & 118 & 0 \\
\hline & \multirow{2}{*}{ Line Operators } & $<50$ & 0 & 0 \\
\hline & & $50+$ & 0 & 0 \\
\hline
\end{tabular}


Figure 12. Rates for Selected Diagnostic Categories by Job Category, Gender, and Age (Continued)

\begin{tabular}{|c|c|c|c|c|}
\hline \multirow{2}{*}{ Injury } & \multicolumn{4}{|c|}{ Rate per 1,000} \\
\hline & Job Category & Age & Men & Women \\
\hline & \multirow{2}{*}{ Professional } & $<50$ & 6 & 16 \\
\hline & & $50+$ & 9 & 79 \\
\hline & \multirow{2}{*}{ Administrative Support } & $<50$ & 71 & 7 \\
\hline & & $50+$ & 0 & 16 \\
\hline & \multirow{2}{*}{ Technical Support } & $<50$ & 42 & 53 \\
\hline & & $50+$ & 39 & 45 \\
\hline & \multirow{2}{*}{ Service } & $<50$ & 100 & 37 \\
\hline & & $50+$ & 80 & 211 \\
\hline & \multirow{2}{*}{ Security and Fire } & $<50$ & 54 & 0 \\
\hline & & $50+$ & 36 & 0 \\
\hline & \multirow{2}{*}{ Crafts } & $<50$ & 141 & 0 \\
\hline & & $50+$ & 118 & 0 \\
\hline & \multirow{2}{*}{ Line Operators } & $<50$ & 0 & 0 \\
\hline & & $50+$ & 0 & 0 \\
\hline
\end{tabular}

\section{Time Trends}

Figure 13. Age-Adjusted Rates for All Diagnoses Combined Among Women and Men from 2006 to 2010*

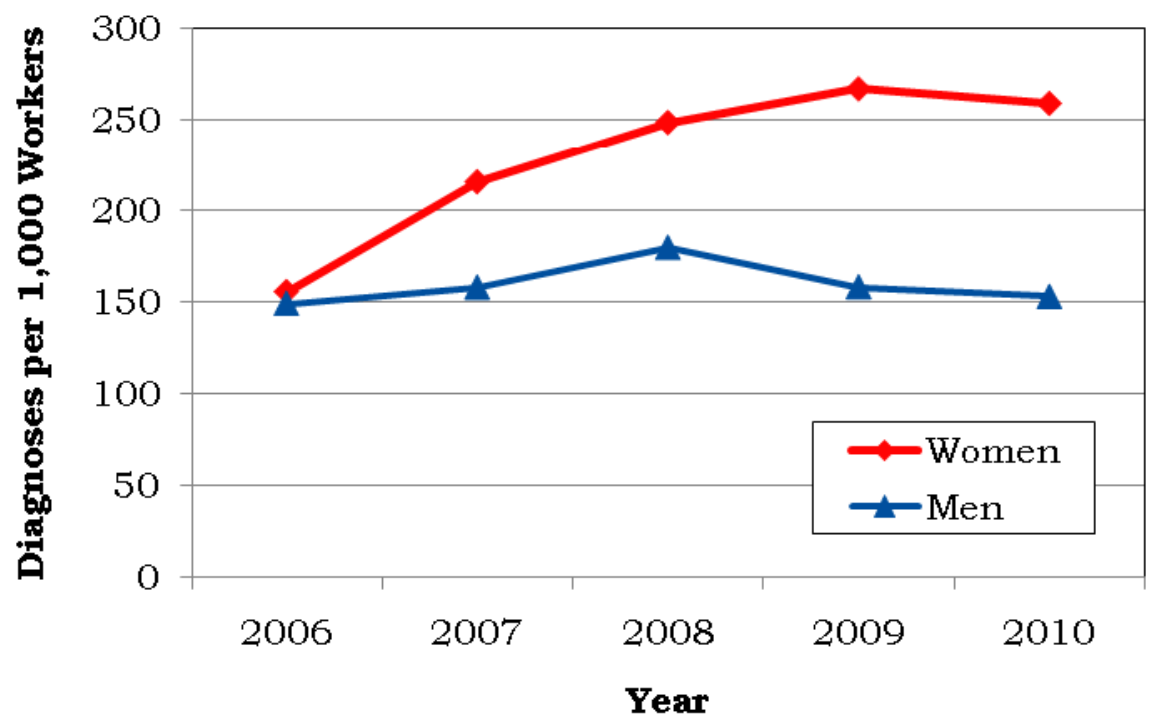

*Standardized to age distribution of 2000 U.S. population. 
Figure 14. Age-Adjusted Rates for Selected Diagnostic Categories Among Women and Men from 2006 to 2010*

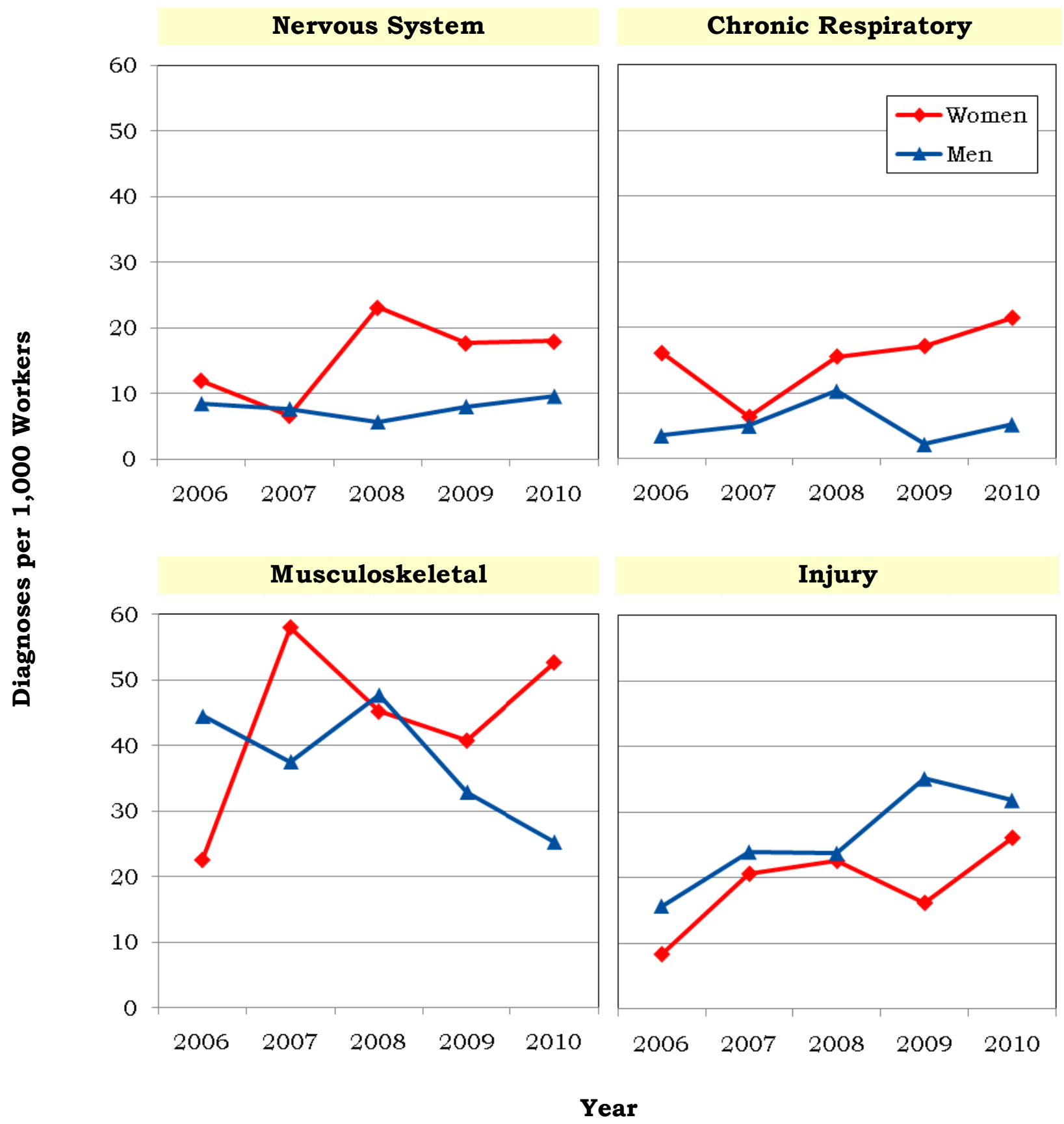

*Standardized to age distribution of 2000 U.S. population. 
Figure 15. Age-Adjusted Rates for All Diagnoses Combined Among Women and Men by Job Category from 2006 to 2010*

Professional

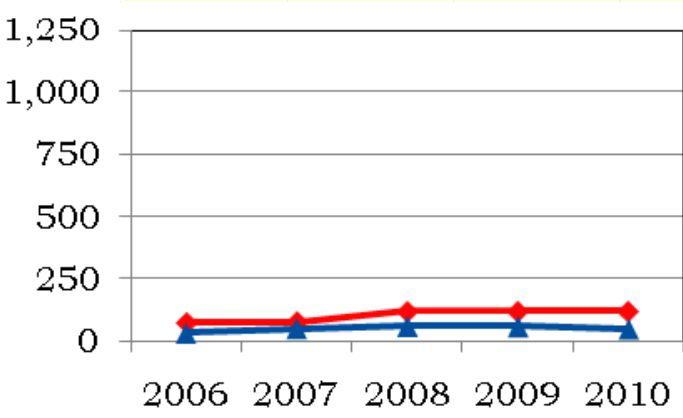

Administrative Support

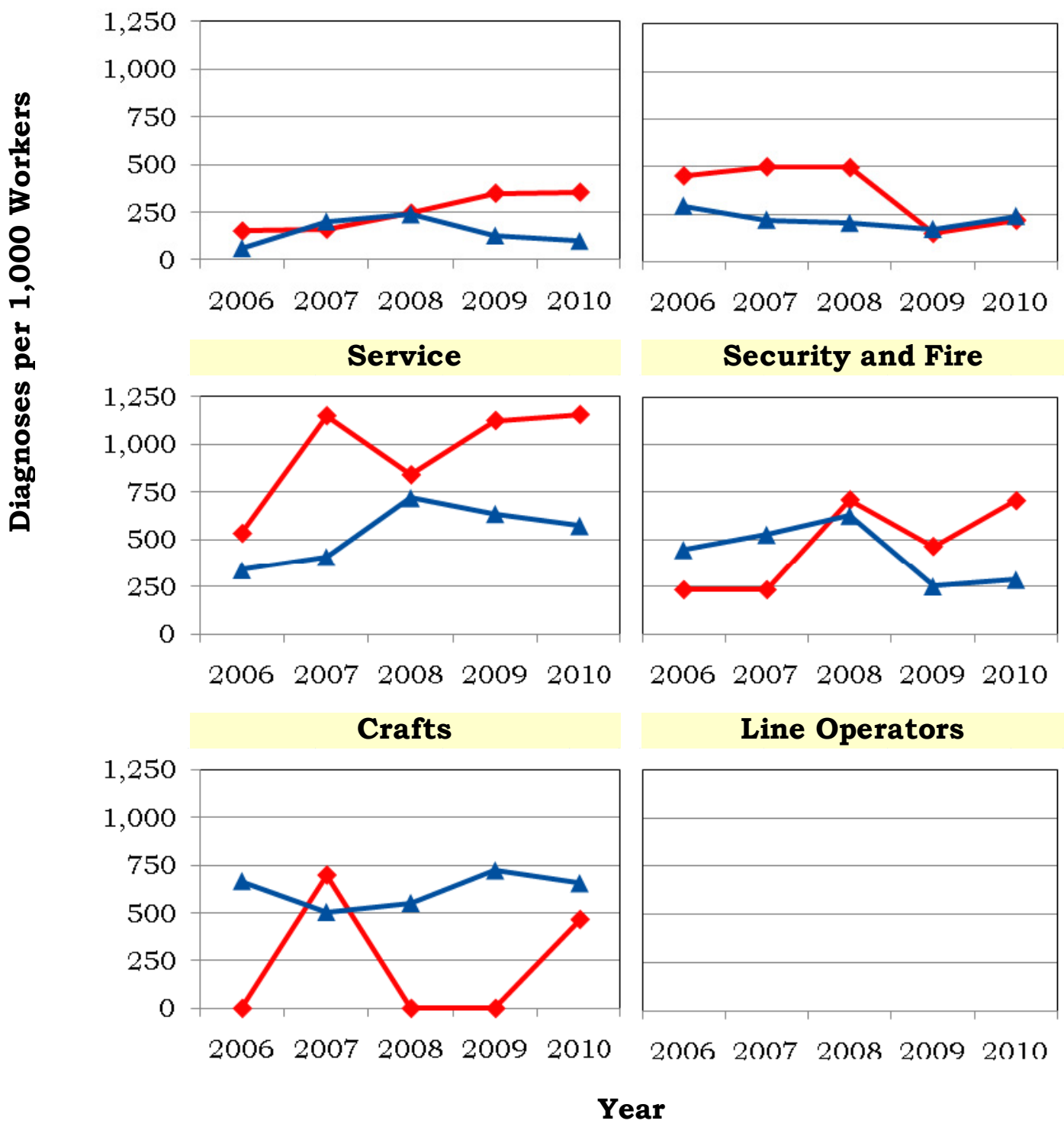

*Standardized to age distribution of 2000 U.S. population. 


\section{Sentinel Health Events for Occupations (SHEOs)}

An occupational sentinel health event (SHEO) is a disease, disability, or death that is likely to be occupationally related. Although sentinel health events may indicate an occupational exposure, many may result from nonoccupational exposures. Sentinel health events are therefore assessed in two categories:

Definite Sentinel Health Events: Diseases that are unlikely to occur in the absence of an occupational exposure (e.g., asbestosis).

Possible Sentinel Health Events: Diseases that may be occupational but can also occur in the absence of an occupational exposure (e.g., lung cancer or carpal tunnel syndrome).

Figure 16. Characteristics of SHEOs by Gender

\begin{tabular}{|l|c|c|c|c|}
\hline \multirow{2}{*}{} & \multicolumn{2}{|c|}{$\begin{array}{c}\text { Total Number of } \\
\text { SHEO Diagnoses }\end{array}$} & \multicolumn{2}{c|}{$\begin{array}{c}\text { Total Number of } \\
\text { Days Absent }\end{array}$} \\
\cline { 2 - 5 } & Men & Women & Men & W omen \\
\hline Definite & 35 & 18 & 1,393 & 678 \\
\hline Possible & 8 & 3 & 104 & 27 \\
\hline Total & 43 & 21 & 1,497 & 705 \\
\hline
\end{tabular}

Figure 17. SHEO Diagnoses by Gender

\begin{tabular}{|l|c|c|}
\hline \multirow{2}{*}{ Diagnoses } & \multicolumn{2}{c|}{ Gender } \\
\cline { 2 - 3 } & Women & M en \\
\hline Carpal Tunnel Syndrome & 7 & 6 \\
\hline M usculoskeletal Conditions & 5 & 13 \\
\hline Injuries & 5 & 18 \\
\hline Other Conditions & 4 & 6 \\
\hline
\end{tabular}


Occupational Safety and Health Administration (OSHA)-Recordable Events

Figure 18. OSHA-Recordable Events by Gender and Age

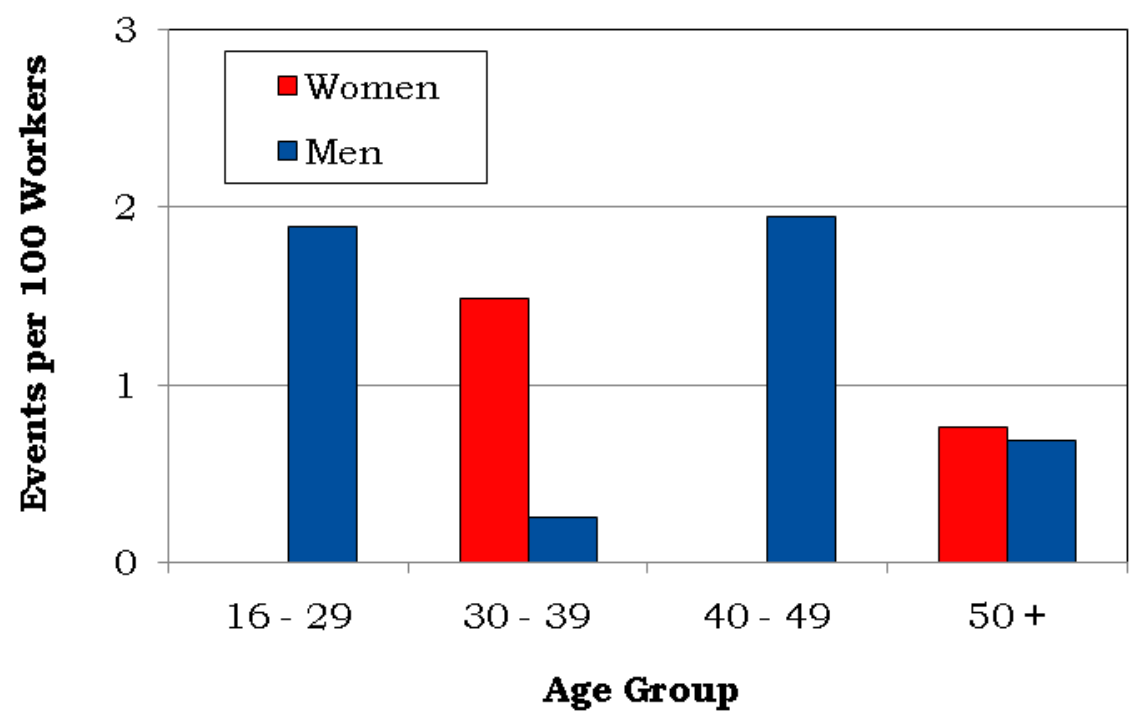

Figure 19. OSHA-Recordable Events by Job Category and Gender

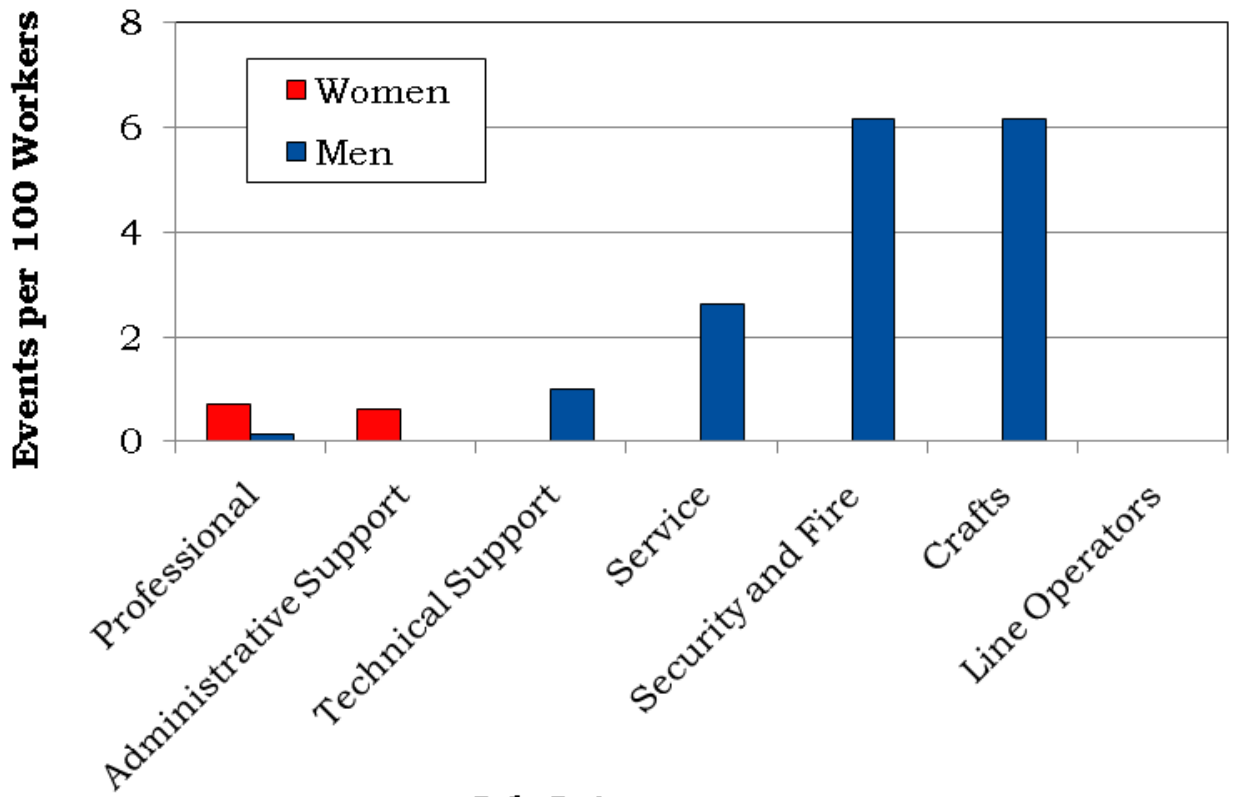

Job Category 


\section{Diagnostic and Accident Categories for OSHA-Recordable Events}

Figure 20. OSHA-Recordable Diagnoses by Diagnostic Category and Gender

\begin{tabular}{|l|c|c|}
\hline \multirow{2}{*}{ Diagnostic Category } & \multicolumn{2}{c|}{ Gender } \\
\cline { 2 - 3 } & Women & M en \\
\hline Musculoskeletal & $\mathbf{0}$ & $\mathbf{1}$ \\
\hline Nervous System & $\mathbf{0}$ & $\mathbf{1}$ \\
\hline Injury & $\mathbf{5}$ & $\mathbf{2 2}$ \\
\hline Fractures - Upper Limb & $\mathbf{0}$ & $\mathbf{1}$ \\
\hline Back Sprains \& Strains & $\mathbf{0}$ & $\mathbf{1}$ \\
\hline Other Sprains \& Strains & $\mathbf{0}$ & $\mathbf{2}$ \\
\hline Open Wounds - Head, Neck, Trunk & $\mathbf{0}$ & $\mathbf{1}$ \\
\hline Open Wounds - Upper Limb & $\mathbf{0}$ & $\mathbf{2}$ \\
\hline Superficial Injuries & $\mathbf{0}$ & $\mathbf{1}$ \\
\hline Crushing Injuries & $\mathbf{0}$ & $\mathbf{1}$ \\
\hline Burns & $\mathbf{1}$ & $\mathbf{1}$ \\
\hline Unspecified Injuries & $\mathbf{3}$ & $\mathbf{1 2}$ \\
\hline Adverse Reactions to Non-Medical Substances & $\mathbf{1}$ & $\mathbf{0}$ \\
\hline
\end{tabular}

Figure 21. OSHA-Recordable Accidents by Type and Gender

\begin{tabular}{|l|c|c|}
\hline \multirow{2}{*}{ Accident Category } & \multicolumn{2}{c|}{ Gender } \\
\cline { 2 - 3 } & Women & Men \\
\cline { 2 - 3 } & $\begin{array}{c}\text { Number of } \\
\text { Accidents }\end{array}$ & $\begin{array}{c}\text { Number of } \\
\text { Accidents }\end{array}$ \\
\hline Falls & $\mathbf{2}$ & $\mathbf{5}$ \\
\hline Natural/Environmental Factors & $\mathbf{1}$ & $\mathbf{0}$ \\
\hline Other Accidents & $\mathbf{0}$ & $\mathbf{1 0}$ \\
\hline Cutting/Piercing Instrument/Object & $\mathbf{0}$ & $\mathbf{1}$ \\
\hline Hot, Corrosive, or Caustic Material/Steam & $\mathbf{0}$ & $\mathbf{1}$ \\
\hline Overexertion/Strenuous Movements & $\mathbf{0}$ & $\mathbf{6}$ \\
\hline Repetitive Trauma & $\mathbf{0}$ & $\mathbf{1}$ \\
\hline Other and Unspecified Accidents & $\mathbf{0}$ & $\mathbf{1}$ \\
\hline Total & $\mathbf{3}$ & $\mathbf{1 5}$ \\
\hline
\end{tabular}




\section{Rates of OSHA-Recordable Events}

Figure 22. OSHA-Recordable Rates by Age and Job Categories Among Women, All Diagnoses Combined

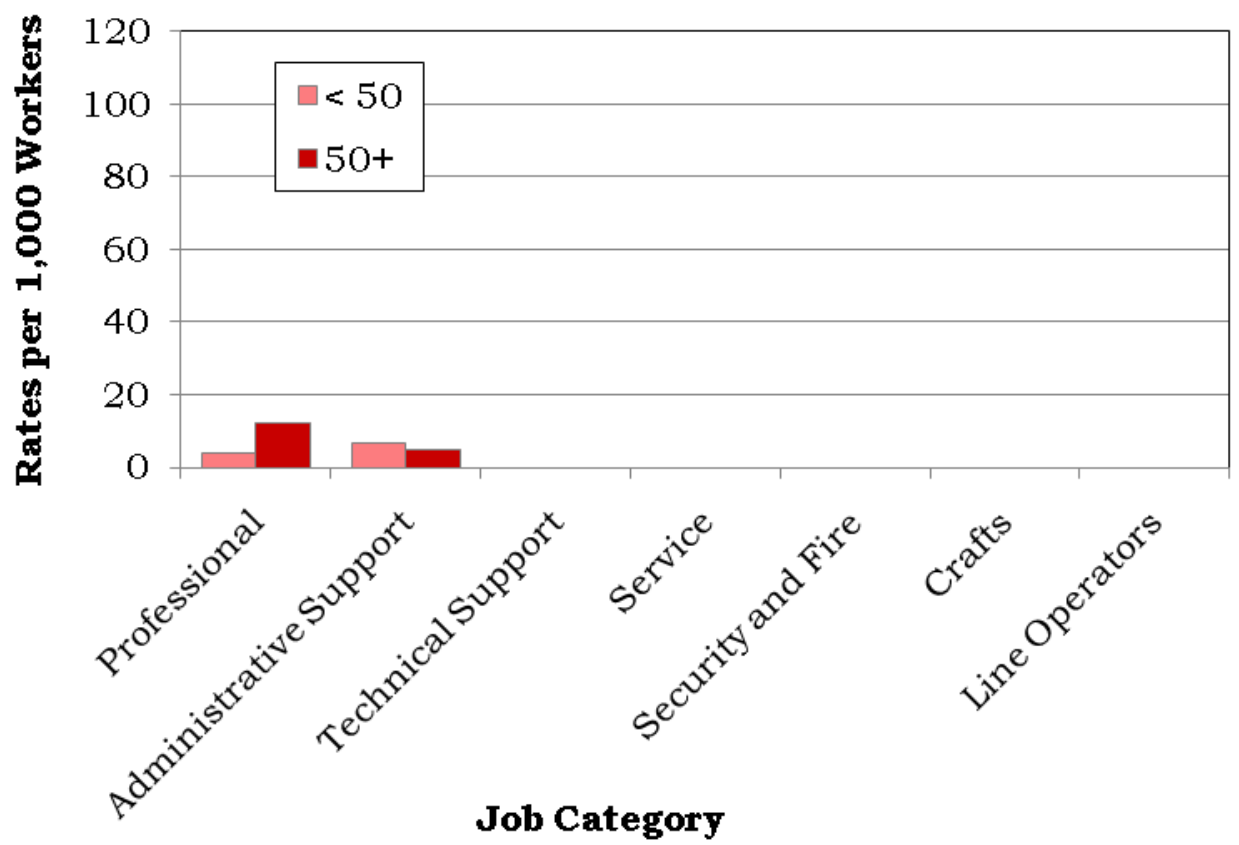

Figure 23. OSHA-Recordable Rates by Age and Job Categories Among Men, All Diagnoses Combined

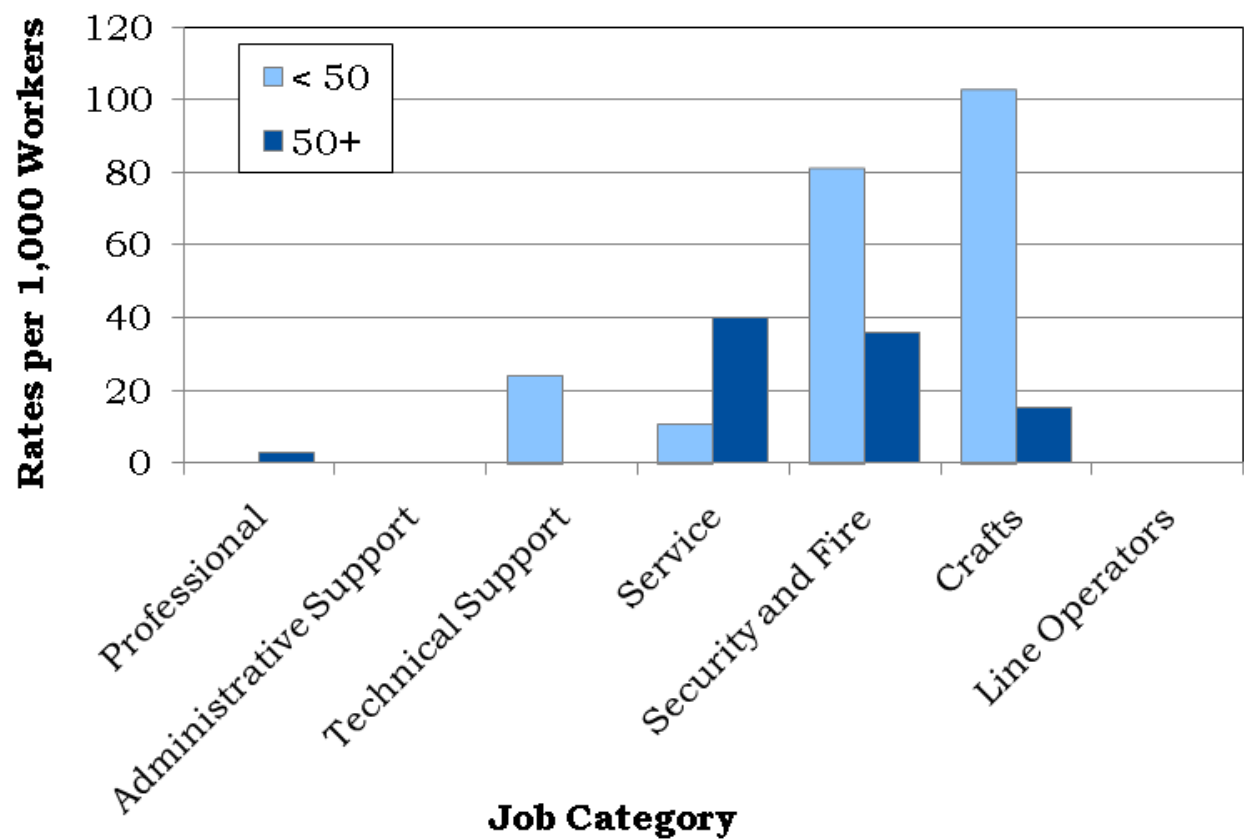




\section{Time Trends for OSHA-Recordable Events}

Figure 24. Age-Adjusted Rates for All OSHA-Recordable Diagnoses Combined Among Women and Men by Job Category from 2006 to 2010*

Professional

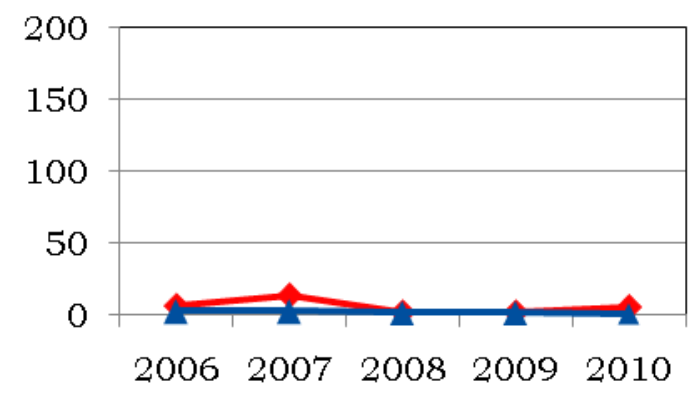

Administrative Support
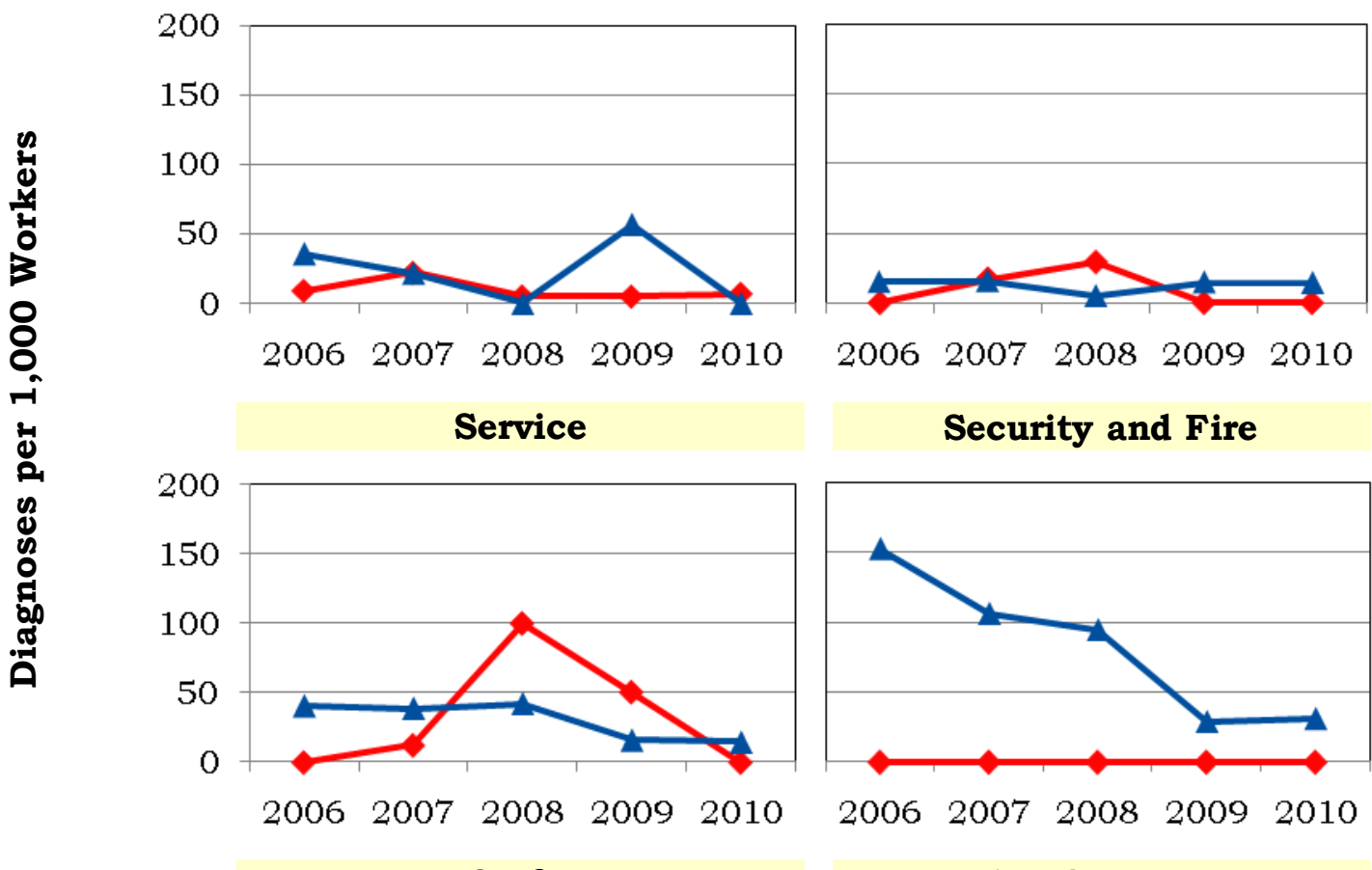

Crafts
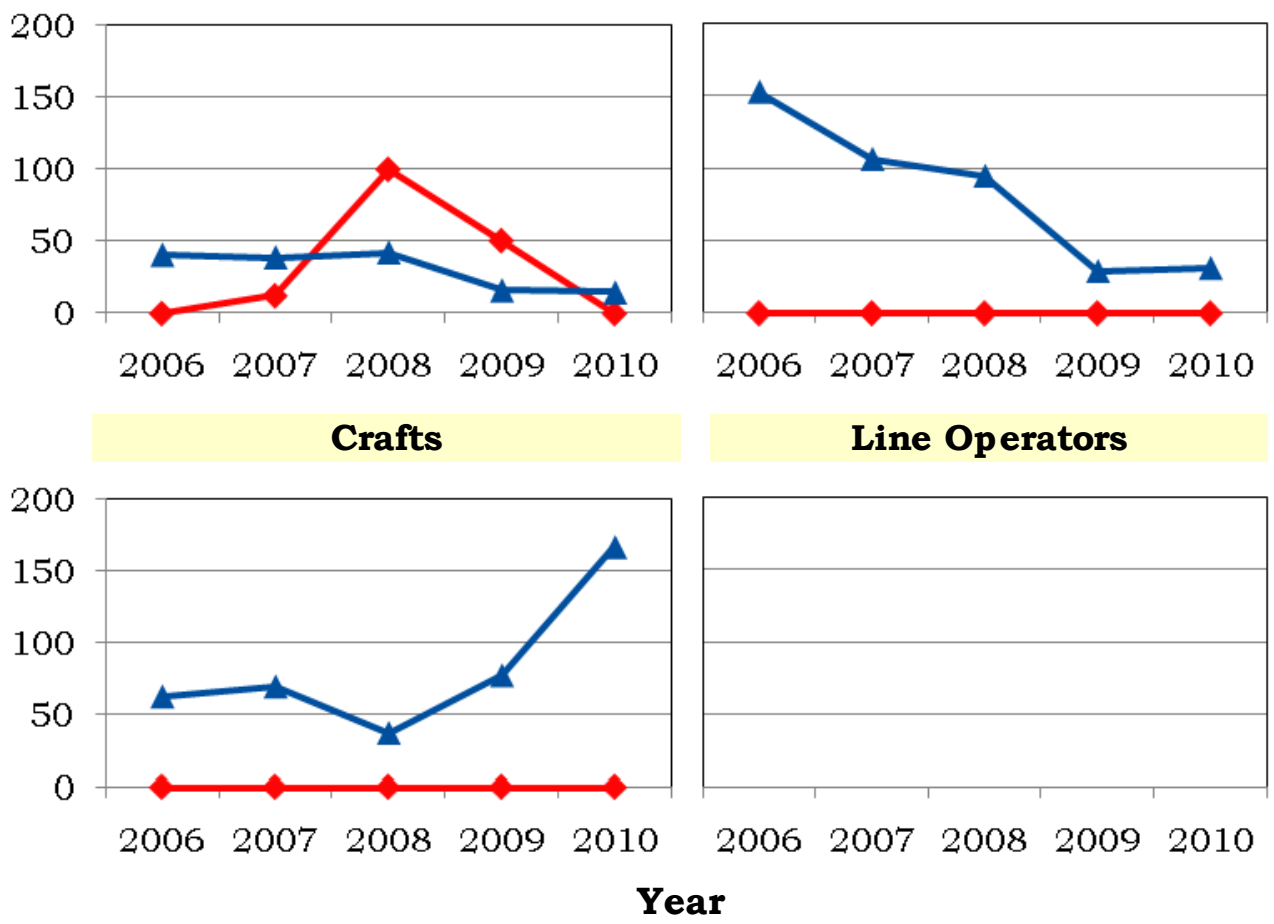

*Standardized to age distribution of 2000 U.S. population. 
Appendices 
Brookhaven National Laboratory 2010

Absence Data

Appendix A. Work Force by Gender, Age, and Job Category

\begin{tabular}{|c|c|c|c|c|c|c|c|c|c|c|c|}
\hline \multirow{3}{*}{ Job Category } & \multicolumn{5}{|c|}{ Women } & \multicolumn{5}{|c|}{ Men } & \multirow[b]{3}{*}{ TOTAL } \\
\hline & \multicolumn{4}{|c|}{ Age Group } & \multirow[b]{2}{*}{ TOTAL } & \multicolumn{4}{|c|}{ Age Group } & \multirow[b]{2}{*}{ TOTAL } & \\
\hline & $16-29$ & 30 - 39 & $40-49$ & $50+$ & & $16-29$ & 30 - 39 & $40-49$ & $50+$ & & \\
\hline Professional & 46 & 81 & 126 & 165 & 418 & 94 & 330 & 368 & 695 & 1,487 & 1,905 \\
\hline Administrative Support & 15 & 43 & 80 & 183 & 321 & 7 & 5 & 2 & 32 & 46 & 367 \\
\hline Technical Support & 3 & 7 & 9 & 22 & 41 & 35 & 30 & 100 & 232 & 397 & 438 \\
\hline Service & 1 & 3 & 23 & 19 & 46 & 15 & 14 & 61 & 100 & 190 & 236 \\
\hline Security and Fire & 2 & 0 & 2 & 2 & 6 & 4 & 4 & 29 & 28 & 65 & 71 \\
\hline Crafts & 0 & 0 & 2 & 1 & 3 & 4 & 20 & 54 & 68 & 146 & 149 \\
\hline TOTAL & 67 & 134 & 242 & 392 & 835 & 159 & 403 & 614 & 1,155 & 2,331 & 3,166 \\
\hline
\end{tabular}

Appendix B. Age Distribution of the Work Force by Gender

\begin{tabular}{|l|r|r|r|r|r|r|r|r|}
\hline \multirow{3}{*}{ Year } & \multicolumn{9}{|c|}{ Women } & \multicolumn{4}{|c|}{ Men } \\
\cline { 2 - 9 } & \multicolumn{1}{|c|}{ Percent Distribution by Age Group } & \multicolumn{1}{c|}{ Percent Distribution by Age Group } \\
\cline { 2 - 9 } & $\mathbf{1 6}-\mathbf{2 9}$ & $\mathbf{3 0}-\mathbf{3 9}$ & $\mathbf{4 0}-\mathbf{4 9}$ & $\mathbf{5 0}+$ & $\mathbf{1 6}-\mathbf{2 9}$ & $\mathbf{3 0}-\mathbf{3 9}$ & $\mathbf{4 0}-\mathbf{4 9}$ & $\mathbf{5 0}+$ \\
\hline $\mathbf{1 9 9 4}$ & 19.78 & 30.12 & 28.60 & 21.50 & 9.31 & 29.13 & 26.54 & 35.02 \\
\hline $\mathbf{1 9 9 5}$ & 16.30 & 31.02 & 29.13 & 23.55 & 7.77 & 28.63 & 28.31 & 35.29 \\
\hline $\mathbf{1 9 9 6}$ & 14.94 & 31.24 & 29.55 & 24.27 & 7.53 & 27.59 & 30.29 & 34.59 \\
\hline $\mathbf{1 9 9 7}$ & 13.73 & 28.49 & 31.56 & 26.22 & 6.34 & 27.74 & 29.60 & 36.32 \\
\hline $\mathbf{1 9 9 8}$ & 12.57 & 25.62 & 33.25 & 28.55 & 6.55 & 25.61 & 31.19 & 36.65 \\
\hline $\mathbf{1 9 9 9}$ & 10.41 & 24.16 & 33.97 & 31.46 & 5.46 & 23.81 & 32.72 & 38.02 \\
\hline $\mathbf{2 0 0 0}$ & 9.70 & 23.95 & 34.49 & 31.86 & 5.70 & 23.03 & 34.39 & 36.87 \\
\hline $\mathbf{2 0 0 1}$ & 9.61 & 22.63 & 36.01 & 31.75 & 5.93 & 20.97 & 35.70 & 37.40 \\
\hline $\mathbf{2 0 0 2}$ & 8.86 & 22.39 & 36.04 & 32.72 & 5.51 & 20.04 & 36.49 & 37.96 \\
\hline $\mathbf{2 0 0 3}$ & 9.14 & 20.90 & 36.42 & 33.54 & 4.97 & 18.22 & 37.06 & 39.74 \\
\hline $\mathbf{2 0 0 4}$ & 9.64 & 20.05 & 35.55 & 34.77 & 4.96 & 17.70 & 37.07 & 40.27 \\
\hline $\mathbf{2 0 0 5}$ & 7.82 & 18.33 & 34.77 & 39.08 & 5.01 & 16.64 & 34.53 & 43.82 \\
\hline $\mathbf{2 0 0 6}$ & 9.08 & 15.75 & 34.18 & 40.99 & 5.14 & 16.79 & 32.15 & 45.93 \\
\hline $\mathbf{2 0 0 7}$ & 8.38 & 17.53 & 31.19 & 42.91 & 5.23 & 16.96 & 31.49 & 46.32 \\
\hline $\mathbf{2 0 0 8}$ & 7.92 & 17.61 & 30.31 & 44.15 & 5.33 & 17.21 & 29.61 & 47.85 \\
\hline $\mathbf{2 0 0 9}$ & 8.16 & 16.56 & 29.61 & 45.67 & 6.04 & 17.50 & 27.89 & 48.57 \\
\hline $\mathbf{2 0 1 0}$ & 8.02 & 16.05 & 28.98 & 46.95 & 6.82 & 17.29 & 26.34 & 49.55 \\
\hline
\end{tabular}


Brookhaven National Laboratory 2010

Absence Data

Appendix C. Total Number of Workers Who Reported at Least One Absence by Gender, Age, and Job Category*

\begin{tabular}{|c|c|c|c|c|c|c|c|c|c|c|c|}
\hline \multirow{3}{*}{ Job Category } & \multicolumn{5}{|c|}{ Women } & \multicolumn{5}{|c|}{ Men } & \multirow[b]{3}{*}{ TOTAL } \\
\hline & \multicolumn{4}{|c|}{ Age Group } & \multirow[b]{2}{*}{ TOTAL } & \multicolumn{4}{|c|}{ Age Group } & \multirow[b]{2}{*}{ TOTAL } & \\
\hline & $16-29$ & 30 - 39 & $40-49$ & $50+$ & & $16-29$ & 30 - 39 & $40-49$ & $50+$ & & \\
\hline Professional & 0 & 6 & 5 & 25 & 36 & 3 & 7 & 18 & 32 & 60 & 96 \\
\hline Administrative Support & 2 & 10 & 11 & 27 & 50 & 1 & 0 & 0 & 5 & 6 & 56 \\
\hline Technical Support & 0 & 3 & 0 & 2 & 5 & 2 & 5 & 10 & 35 & 52 & 57 \\
\hline Service & 0 & 2 & 10 & 11 & 23 & 4 & 4 & 14 & 35 & 57 & 80 \\
\hline Security and Fire & 1 & 0 & 1 & 1 & 3 & 1 & 0 & 9 & 8 & 18 & 21 \\
\hline Crafts & 0 & 0 & 1 & 1 & 2 & 2 & 11 & 13 & 28 & 54 & 56 \\
\hline TOTAL & 3 & 21 & 28 & 67 & 119 & 13 & 27 & 64 & 143 & 247 & 366 \\
\hline
\end{tabular}

*Only those job categories and gender/age combinations with at least one absence appear in this table.

Appendix D. Total Number of Absences by Gender, Age, and Job Category*

\begin{tabular}{|c|c|c|c|c|c|c|c|c|c|c|c|}
\hline \multirow{3}{*}{ Job Category } & \multicolumn{5}{|c|}{ Women } & \multicolumn{5}{|c|}{ Men } & \multirow[b]{3}{*}{ TOTAL } \\
\hline & \multicolumn{4}{|c|}{ Age Group } & \multirow[b]{2}{*}{ TOTAL } & \multicolumn{4}{|c|}{ Age Group } & \multirow[b]{2}{*}{ TOTAL } & \\
\hline & $16-29$ & 30 - 39 & $40-49$ & $50+$ & & $16-29$ & 30 - 39 & $40-49$ & $50+$ & & \\
\hline Professional & 0 & 7 & 5 & 29 & 41 & 3 & 8 & 19 & 36 & 66 & 107 \\
\hline Administrative Support & 2 & 16 & 17 & 39 & 74 & 1 & 0 & 0 & 6 & 7 & 81 \\
\hline Technical Support & 0 & 5 & 0 & 2 & 7 & 3 & 6 & 13 & 46 & 68 & 75 \\
\hline Service & 0 & 4 & 15 & 21 & 40 & 5 & 6 & 29 & 52 & 92 & 132 \\
\hline Security and Fire & 1 & 0 & 1 & 2 & 4 & 1 & 0 & 10 & 9 & 20 & 24 \\
\hline Crafts & 0 & 0 & 1 & 2 & 3 & 2 & 15 & 14 & 36 & 67 & 70 \\
\hline TOTAL & 3 & 32 & 39 & 95 & 169 & 15 & 35 & 85 & 185 & 320 & 489 \\
\hline
\end{tabular}

*Only those job categories and gender/age combinations with at least one absence appear in this table. 
Brookhaven National Laboratory 2010

Absence Data

Appendix E. Distribution of the Number of Calendar Days Missed per Absence by Gender and Age*

\begin{tabular}{|c|c|c|c|c|c|c|c|c|c|c|c|}
\hline \multirow{3}{*}{$\begin{array}{c}\text { Number of } \\
\text { Calendar Days }\end{array}$} & \multicolumn{5}{|c|}{ Women } & \multicolumn{5}{|c|}{ Men } & \multirow[b]{3}{*}{ TOTAL } \\
\hline & \multicolumn{4}{|c|}{ Age Group } & \multirow[b]{2}{*}{ TOTAL } & \multicolumn{4}{|c|}{ Age Group } & \multirow[b]{2}{*}{ TOTAL } & \\
\hline & $16-29$ & $30-39$ & $40-49$ & $50+$ & & $16-29$ & 30 - 39 & $40-49$ & $50+$ & & \\
\hline$<15$ & 3 & 24 & 31 & 71 & 129 & 14 & 27 & 66 & 116 & 223 & 352 \\
\hline $15-28$ & 0 & 4 & 4 & 5 & 13 & 1 & 4 & 11 & 24 & 40 & 53 \\
\hline $29-42$ & 0 & 0 & 1 & 3 & 4 & 0 & 1 & 3 & 20 & 24 & 28 \\
\hline $43-56$ & 0 & 0 & 0 & 3 & 3 & 0 & 1 & 1 & 5 & 7 & 10 \\
\hline $57-91$ & 0 & 3 & 2 & 9 & 14 & 0 & 2 & 2 & 7 & 11 & 25 \\
\hline $92-182$ & 0 & 0 & 0 & 3 & 3 & 0 & 0 & 1 & 10 & 11 & 14 \\
\hline $183+$ & 0 & 1 & 1 & 1 & 3 & 0 & 0 & 1 & 3 & 4 & 7 \\
\hline TOTAL & 3 & 32 & 39 & 95 & 169 & 15 & 35 & 85 & 185 & 320 & 489 \\
\hline
\end{tabular}

*Only those gender/age combinations with at least one absence appear in this table. 
Brookhaven National Laboratory 2010

Absence Data

Appendix F. Distribution of the Number of Calendar Days Missed per Absence by Gender and Job Category*

Women

\begin{tabular}{|c|c|c|c|c|c|c|c|}
\hline \multirow{2}{*}{$\begin{array}{c}\text { Number of } \\
\text { Calendar Days }\end{array}$} & \multicolumn{6}{|c|}{ Job Category } & \multirow[b]{2}{*}{ TOTAL } \\
\hline & Professional & $\begin{array}{c}\text { Administrative } \\
\text { Support }\end{array}$ & Technical Support & Service & Security and Fire & Crafts & \\
\hline$<15$ & 27 & 61 & 4 & 30 & 4 & 3 & 129 \\
\hline $15-28$ & 4 & 4 & 3 & 2 & 0 & 0 & 13 \\
\hline $29-42$ & 0 & 3 & 0 & 1 & 0 & 0 & 4 \\
\hline $43-56$ & 1 & 1 & 0 & 1 & 0 & 0 & 3 \\
\hline $57-91$ & 5 & 5 & 0 & 4 & 0 & 0 & 14 \\
\hline $92-182$ & 3 & 0 & 0 & 0 & 0 & 0 & 3 \\
\hline $183+$ & 1 & 0 & 0 & 2 & 0 & 0 & 3 \\
\hline TOTAL & 41 & 74 & 7 & 40 & 4 & 3 & 169 \\
\hline
\end{tabular}

Men

\begin{tabular}{|c|c|c|c|c|c|c|c|}
\hline \multirow{2}{*}{$\begin{array}{l}\text { Number of } \\
\text { Calendar Days }\end{array}$} & \multicolumn{6}{|c|}{ Job Category } & \multirow[b]{2}{*}{ TOTAL } \\
\hline & Professional & $\begin{array}{c}\text { Administrative } \\
\text { Support }\end{array}$ & Technical Support & Service & Security and Fire & Crafts & \\
\hline$<15$ & 37 & 6 & 47 & 68 & 14 & 51 & 223 \\
\hline $15-28$ & 15 & 0 & 6 & 9 & 3 & 7 & 40 \\
\hline $29-42$ & 5 & 1 & 7 & 6 & 1 & 4 & 24 \\
\hline $43-56$ & 3 & 0 & 1 & 1 & 0 & 2 & 7 \\
\hline $57-91$ & 3 & 0 & 2 & 5 & 1 & 0 & 11 \\
\hline $92-182$ & 1 & 0 & 4 & 3 & 1 & 2 & 11 \\
\hline $183+$ & 2 & 0 & 1 & 0 & 0 & 1 & 4 \\
\hline TOTAL & 66 & 7 & 68 & 92 & 20 & 67 & 320 \\
\hline
\end{tabular}

*Only those gender/job category combinations with at least one absence appear in this table. 


\section{Brookhaven National Laboratory 2010}

Absence Data

Appendix G. Number of Diagnoses in Each Diagnostic Category by Gender and Age*

\begin{tabular}{|c|c|c|c|c|c|c|}
\hline & & \multicolumn{5}{|c|}{ Women } \\
\hline & & \multicolumn{4}{|c|}{ Age Group } & \multirow[b]{2}{*}{ TOTAL } \\
\hline & & $16-29$ & $30-39$ & $40-49$ & $50+$ & \\
\hline Diagnostic Category & ICD-9-CM Code & \multirow[b]{2}{*}{0} & \multirow[b]{2}{*}{0} & \multirow[b]{2}{*}{4} & \multirow[b]{2}{*}{2} & \multirow[b]{2}{*}{6} \\
\hline INFECTIOUS \& PARASITIC DISEASES (DIS) & 001-139 & & & & & \\
\hline -Other Bacterial Dis & $030-041$ & 0 & 0 & 0 & 0 & 0 \\
\hline -Viral Dis with Exanthem & $050-059$ & 0 & 0 & 0 & 0 & 0 \\
\hline -Other Viral Dis \& Chlamydiae & 070-079 & 0 & 0 & 3 & 2 & 5 \\
\hline -Rickettsioses \& Other Arthropod & $080-088$ & 0 & 0 & 1 & 0 & 1 \\
\hline -Other Infections \& Parasitic Dis & $130-136$ & 0 & 0 & 0 & 0 & 0 \\
\hline MALIGNANT NEOPLASMS & $\begin{array}{l}140-208 \\
209.0-209.3 \\
230-234\end{array}$ & 0 & 0 & 0 & 2 & 2 \\
\hline -Lip, Oral Cavity, Pharynx & $140-149$ & 0 & 0 & 0 & 0 & 0 \\
\hline -Digestive \& Peritoneal & $150-159$ & 0 & 0 & 0 & 0 & 0 \\
\hline -Respiratory \& Intrathoracic & $160-165$ & 0 & 0 & 0 & 0 & 0 \\
\hline -Bone, Connective Tissue, Skin & $170-173,176$ & 0 & 0 & 0 & 0 & 0 \\
\hline -Breast & 174-175 & 0 & 0 & 0 & 1 & 1 \\
\hline -Genitourinary & 179-189 & 0 & 0 & 0 & 1 & 1 \\
\hline -Other \& Unspecified Sites & $195-199,209.7$ & 0 & 0 & 0 & 0 & 0 \\
\hline -Lymphatic \& Hematopoietic & $200-208$ & 0 & 0 & 0 & 0 & 0 \\
\hline BENIGN \& UNCERTAIN NEOPLASMS & \begin{tabular}{|l|}
$209.4-209.6$ \\
$210-229$, \\
$235-239$
\end{tabular} & 0 & 2 & 2 & 1 & 5 \\
\hline ENDOCRINE/METABOLIC/IMMUNITY & $240-279$ & 0 & 1 & 1 & 1 & 3 \\
\hline -Thyroid Gland Disorders & $240-246$ & 0 & 0 & 0 & 0 & 0 \\
\hline -Other Endocrine Gland Dis & $249-259$ & 0 & 1 & 0 & 1 & 2 \\
\hline -Other Metabolic \& Immunity Disorders & $270-279$ & 0 & 0 & 1 & 0 & 1 \\
\hline MENTAL DISORDERS & $290-319$ & 0 & 4 & 2 & 4 & 10 \\
\hline -Psychoses & 290-299 & 0 & 1 & 0 & 0 & 1 \\
\hline -Non-Psychotic Disorders & $300-302,306-316$ & 0 & 2 & 2 & 3 & 7 \\
\hline -Alcohol Dependence & 303 & 0 & 0 & 0 & 1 & 1 \\
\hline -Drug Dependence & $304-305$ & 0 & 1 & 0 & 0 & 1 \\
\hline NERVOUS SYSTEM (NS) \& SENSE ORGANS & $320-389$ & 0 & 5 & 3 & 9 & 17 \\
\hline -Hereditary/Degenerative Central NS Dis & 330-337 & 0 & 0 & 0 & 0 & 0 \\
\hline -Other Disorders of Central NS & $340-349$ & 0 & 1 & 0 & 1 & 2 \\
\hline -Disorders of Peripheral NS & $350-359$ & 0 & 1 & 1 & 5 & 7 \\
\hline -Disorders of Eye & \begin{tabular}{|l|}
$360-379$ \\
\end{tabular} & 0 & 1 & 0 & 0 & 1 \\
\hline -Diseases of Ear \& Mastoid & $380-389$ & 0 & 2 & 2 & 3 & 7 \\
\hline CIRCULATORY SYSTEM & $390-459$ & 0 & 2 & 1 & 4 & 7 \\
\hline -Hypertensive Dis & $401-405$ & 0 & 2 & 0 & 1 & 3 \\
\hline -Ischemic Heart Dis & $410-414$ & 0 & 0 & 0 & 0 & 0 \\
\hline -Other Heart Dis & $420-429$ & 0 & 0 & 0 & 1 & 1 \\
\hline
\end{tabular}

(Continued)

*Only those diagnostic categories and gender/age combinations with at least one occurrence appear in this table. 


\section{Brookhaven National Laboratory 2010}

\section{Absence Data}

Appendix G. Number of Diagnoses in Each Diagnostic Category by Gender and Age*

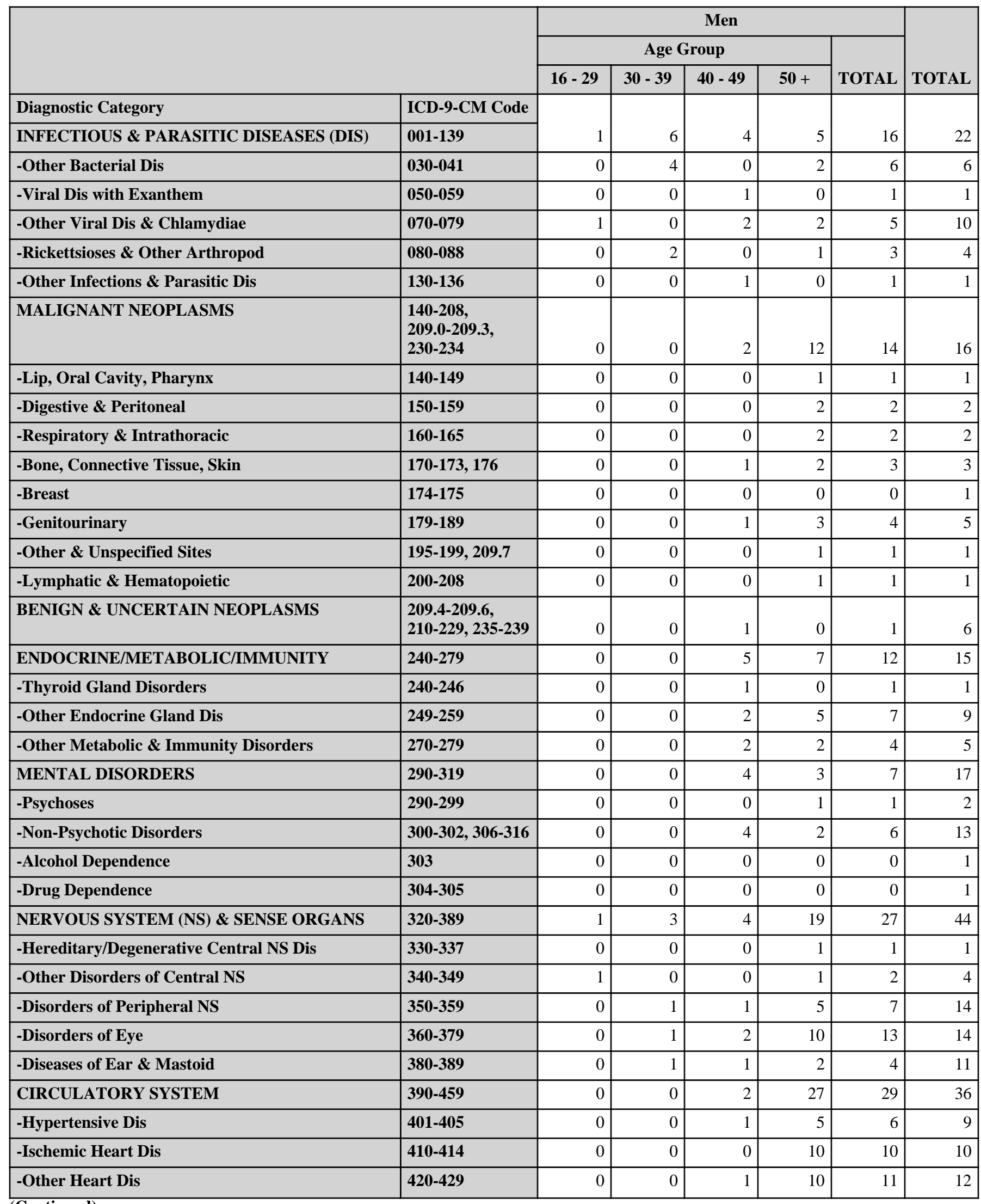

(Continued)

*Only those diagnostic categories and gender/age combinations with at least one occurrence appear in this table. 


\section{Brookhaven National Laboratory 2010}

Absence Data

Appendix G. Number of Diagnoses in Each Diagnostic Category by Gender and Age*

\begin{tabular}{|c|c|c|c|c|c|c|}
\hline & & \multicolumn{5}{|c|}{ Women } \\
\hline & & \multicolumn{4}{|c|}{ Age Group } & \multirow[b]{2}{*}{ TOTAL } \\
\hline & & $16-29$ & $30-39$ & $40-49$ & $50+$ & \\
\hline Diagnostic Category & ICD-9-CM Code & \multirow[b]{2}{*}{0} & \multirow[b]{2}{*}{0} & \multirow[b]{2}{*}{0} & \multirow[b]{2}{*}{1} & \multirow[b]{2}{*}{1} \\
\hline -Dis of Arteries \& Capillaries & $440-449$ & & & & & \\
\hline -Dis of Veins, Lymphatics, Other & $451-459$ & 0 & 0 & 1 & 1 & 2 \\
\hline RESPIRATORY SYSTEM & $460-519$ & 4 & 6 & 15 & 31 & 56 \\
\hline -Acute Respiratory Infections & $460-466$ & 2 & 1 & 2 & 6 & 11 \\
\hline -Other Dis Upper Respiratory Tract & $470-478$ & 2 & 1 & 5 & 9 & 17 \\
\hline -Pneumonia \& Influenza & $480-488$ & 0 & 1 & 1 & 3 & 5 \\
\hline -Chronic Obstructive Dis & $490-496$ & 0 & 3 & 7 & 12 & 22 \\
\hline -Other Respiratory Dis & $510-519$ & 0 & 0 & 0 & 1 & 1 \\
\hline DIGESTIVE SYSTEM & $520-579$ & 0 & 6 & 5 & 2 & 13 \\
\hline -Oral Cavity, Saliva Glands, Jaw & $520-529$ & 0 & 1 & 0 & 0 & 1 \\
\hline -Esophagus, Stomach, Duodenum & $530-538$ & 0 & 0 & 0 & 0 & 0 \\
\hline -Appendicitis & $540-543$ & 0 & 2 & 0 & 0 & 2 \\
\hline -Hernias & $550-553$ & 0 & 0 & 1 & 0 & 1 \\
\hline -Enteritis, Colitis & $555-558$ & 0 & 0 & 3 & 1 & 4 \\
\hline -Other Intestinal Dis & $560-569$ & 0 & 2 & 1 & 0 & 3 \\
\hline -Other Digestive Dis & $570-579$ & 0 & 1 & 0 & 1 & 2 \\
\hline GENITOURINARY SYSTEM & $580-629$ & 0 & 4 & 5 & 4 & 13 \\
\hline -Other Urinary Dis & $590-599$ & 0 & 2 & 0 & 1 & 3 \\
\hline -Male Genital Organ Dis & $600-608$ & 0 & 0 & 0 & 0 & 0 \\
\hline -Breast Disorders & $610-612$ & 0 & 1 & 0 & 3 & 4 \\
\hline -Other Female Disorders & $617-629$ & 0 & 1 & 5 & 0 & 6 \\
\hline SKIN AND SUBCUTANEOUS TISSUE & $680-709$ & 0 & 0 & 0 & 2 & 2 \\
\hline -Infections & $680-686$ & 0 & 0 & 0 & 1 & 1 \\
\hline -Other Inflammatory Conditions & $690-698$ & 0 & 0 & 0 & 1 & 1 \\
\hline -Other & \begin{tabular}{|l|}
$700-709$ \\
\end{tabular} & 0 & 0 & 0 & 0 & 0 \\
\hline $\begin{array}{l}\text { MUSCULOSKELETAL \& CONNECTIVE } \\
\text { TISSUE }\end{array}$ & $710-739$ & 0 & 12 & 11 & 29 & 52 \\
\hline -Arthropathies & 710-719 & 0 & 4 & 3 & 14 & 21 \\
\hline -Dorsopathies & $720-724$ & 0 & 6 & 8 & 6 & 20 \\
\hline -Rheumatism, Excluding Back & $725-729$ & 0 & 2 & 0 & 6 & 8 \\
\hline -Other Dis \& Acquired Deformities & 730-739 & 0 & 0 & 0 & 3 & 3 \\
\hline CONGENITAL ANOMALIES & $740-759$ & 0 & 0 & 0 & 1 & 1 \\
\hline $\begin{array}{l}\text { SYMPTOMS, SIGNS, \& ILL-DEFINED } \\
\text { CONDITIONS }\end{array}$ & 780-799 & 0 & 7 & 7 & 16 & 30 \\
\hline -Symptoms & 780-789 & 0 & 7 & 7 & 15 & 29 \\
\hline -Non-Specific Abnormal Findings & $790-796$ & 0 & 0 & 0 & 1 & 1 \\
\hline INJURY \& POISONING & $800-999$ & 0 & 5 & 2 & 21 & 28 \\
\hline
\end{tabular}

(Continued)

*Only those diagnostic categories and gender/age combinations with at least one occurrence appear in this table. 


\section{Brookhaven National Laboratory 2010}

\section{Absence Data}

Appendix G. Number of Diagnoses in Each Diagnostic Category by Gender and Age*

\begin{tabular}{|c|c|c|c|c|c|c|c|}
\hline & & \multicolumn{5}{|c|}{ Men } & \multirow[b]{3}{*}{ TOTAL } \\
\hline & & \multicolumn{4}{|c|}{ Age Group } & \multirow[b]{2}{*}{ TOTAL } & \\
\hline & & $16-29$ & $30-39$ & $40-49$ & $50+$ & & \\
\hline -Dis of Arteries \& Capillaries & $440-449$ & 0 & 0 & 0 & 0 & 0 & 1 \\
\hline -Dis of Veins, Lymphatics, Other & $451-459$ & 0 & 0 & 0 & 2 & 2 & 4 \\
\hline -Acute Respiratory Infections & $460-466$ & 1 & 5 & 7 & 15 & 28 & 39 \\
\hline -Other Dis Upper Respiratory Tract & $470-478$ & 2 & 1 & 2 & 4 & 9 & 26 \\
\hline -Pneumonia \& Influenza & $480-488$ & 0 & 1 & 0 & 3 & 4 & 9 \\
\hline -Chronic Obstructive Dis & $490-496$ & 1 & 2 & 3 & 6 & 12 & 34 \\
\hline -Esophagus, Stomach, Duodenum & $530-538$ & 0 & 0 & 0 & 1 & 1 & 1 \\
\hline -Appendicitis & $540-543$ & 2 & 0 & 0 & 1 & 3 & 5 \\
\hline -Hernias & $550-553$ & 0 & 1 & 2 & 10 & 13 & 14 \\
\hline -Enteritis, Colitis & $555-558$ & 1 & 7 & 5 & 5 & 18 & 22 \\
\hline -Other Intestinal Dis & $560-569$ & 0 & 2 & 1 & 6 & 9 & 12 \\
\hline -Other Digestive Dis & $570-579$ & 0 & 0 & 1 & 4 & 5 & 7 \\
\hline GENITOURINARY SYSTEM & $580-629$ & 0 & 0 & 4 & 5 & 9 & 22 \\
\hline -Other Urinary Dis & $590-599$ & 0 & 0 & 3 & 4 & 7 & 10 \\
\hline -Other Inflammatory Conditions & $690-698$ & 0 & 0 & 1 & 1 & 2 & 3 \\
\hline -Other & 700-709 & 0 & 0 & 0 & 3 & 3 & 3 \\
\hline $\begin{array}{l}\text { MUSCULOSKELETAL \& CONNECTIVE } \\
\text { TISSUE }\end{array}$ & $710-739$ & 1 & 5 & 25 & 49 & 80 & 132 \\
\hline -Arthropathies & 710-719 & 0 & 3 & 13 & 17 & 33 & 54 \\
\hline -Dorsopathies & $720-724$ & 1 & 2 & 9 & 24 & 36 & 56 \\
\hline -Rheumatism, Excluding Back & 725-729 & 0 & 0 & 2 & 8 & 10 & 18 \\
\hline -Other Dis \& Acquired Deformities & $730-739$ & 0 & 0 & 1 & 0 & 1 & 4 \\
\hline CONGENITAL ANOMALIES & 740-759 & 0 & 0 & 0 & 0 & 0 & 1 \\
\hline $\begin{array}{l}\text { SYMPTOMS, SIGNS, \& ILL-DEFINED } \\
\text { CONDITIONS }\end{array}$ & $780-799$ & 0 & 7 & 9 & 17 & 33 & 63 \\
\hline -Symptoms & 780-789 & 0 & 5 & 9 & 16 & 30 & 59 \\
\hline -Non-Specific Abnormal Findings & $790-796$ & 0 & 2 & 0 & 1 & 3 & 4 \\
\hline INJURY \& POISONING & $800-999$ & 8 & 6 & 21 & 32 & 67 & 95 \\
\hline
\end{tabular}

(Continued)

*Only those diagnostic categories and gender/age combinations with at least one occurrence appear in this table. 
Brookhaven National Laboratory 2010

Absence Data

Appendix G. Number of Diagnoses in Each Diagnostic Category by Gender and Age*

\begin{tabular}{|c|c|c|c|c|c|c|}
\hline & & \multicolumn{5}{|c|}{ Women } \\
\hline & & \multicolumn{4}{|c|}{ Age Group } & \multirow[b]{2}{*}{ TOTAL } \\
\hline & & $16-29$ & $30-39$ & $40-49$ & $50+$ & \\
\hline Diagnostic Category & ICD-9-CM Code & \multirow[b]{2}{*}{0} & \multirow[b]{2}{*}{1} & \multirow[b]{2}{*}{0} & \multirow[b]{2}{*}{0} & \multirow[b]{2}{*}{1} \\
\hline -Fracture - Neck, Trunk & $805-809$ & & & & & \\
\hline -Fracture - Upper Limb & $810-819$ & 0 & 0 & 1 & 4 & 5 \\
\hline -Fracture - Lower Limb & $820-829$ & 0 & 0 & 1 & 3 & 4 \\
\hline -Sprains \& Strains - Back & 846-847 & 0 & 1 & 0 & 0 & 1 \\
\hline -Sprains \& Strains - Other & $840-845,848$ & 0 & 0 & 0 & 5 & 5 \\
\hline -Intracranial Injury & $850-854$ & 0 & 0 & 0 & 0 & 0 \\
\hline -Internal Injury - Thorax, Abdomen, Pelvis & $860-869$ & 0 & 0 & 0 & 0 & 0 \\
\hline -Open Wound - Head, Neck, Trunk & 870-879 & 0 & 0 & 0 & 1 & 1 \\
\hline -Open Wound - Upper Limb & 880-887 & 0 & 0 & 0 & 1 & 1 \\
\hline -Injury to Blood Vessels & 900-904 & 0 & 0 & 0 & 0 & 0 \\
\hline -Late Effects of Accident & $905-909$ & 0 & 0 & 0 & 1 & 1 \\
\hline -Contusion & $920-924$ & 0 & 0 & 0 & 4 & 4 \\
\hline -Crushing Injury & $925-929$ & 0 & 0 & 0 & 0 & 0 \\
\hline -Foreign Body Entering Orifice & 930-939 & 0 & 0 & 0 & 0 & 0 \\
\hline -Unspecified Effects - External Causes & $990-995$ & 0 & 2 & 0 & 1 & 3 \\
\hline -Complications of Surgical/Medical Care & 996-999 & 0 & 1 & 0 & 1 & 2 \\
\hline $\begin{array}{l}\text { HEALTH STATUS/HEALTH SERVICE } \\
\text { CONTACT }\end{array}$ & V01-V89 & 0 & 7 & 3 & 1 & 11 \\
\hline -Personal \& Family History & V10-V19 & 0 & 0 & 1 & 0 & 1 \\
\hline -Health Services Reproduction/Development & V20-V29 & 0 & 7 & 0 & 0 & 7 \\
\hline -Health Status & V40-V49 & 0 & 0 & 0 & 0 & 0 \\
\hline -Specific Procedures/Aftercare & V50-V59 & 0 & 0 & 2 & 0 & 2 \\
\hline -Other Circumstances & V60-V69 & 0 & 0 & 0 & 1 & 1 \\
\hline -Examination \& Investigation & V70-V82 & 0 & 0 & 0 & 0 & 0 \\
\hline
\end{tabular}

*Only those diagnostic categories and gender/age combinations with at least one occurrence appear in this table. 


\section{Brookhaven National Laboratory 2010}

Absence Data

Appendix G. Number of Diagnoses in Each Diagnostic Category by Gender and Age*

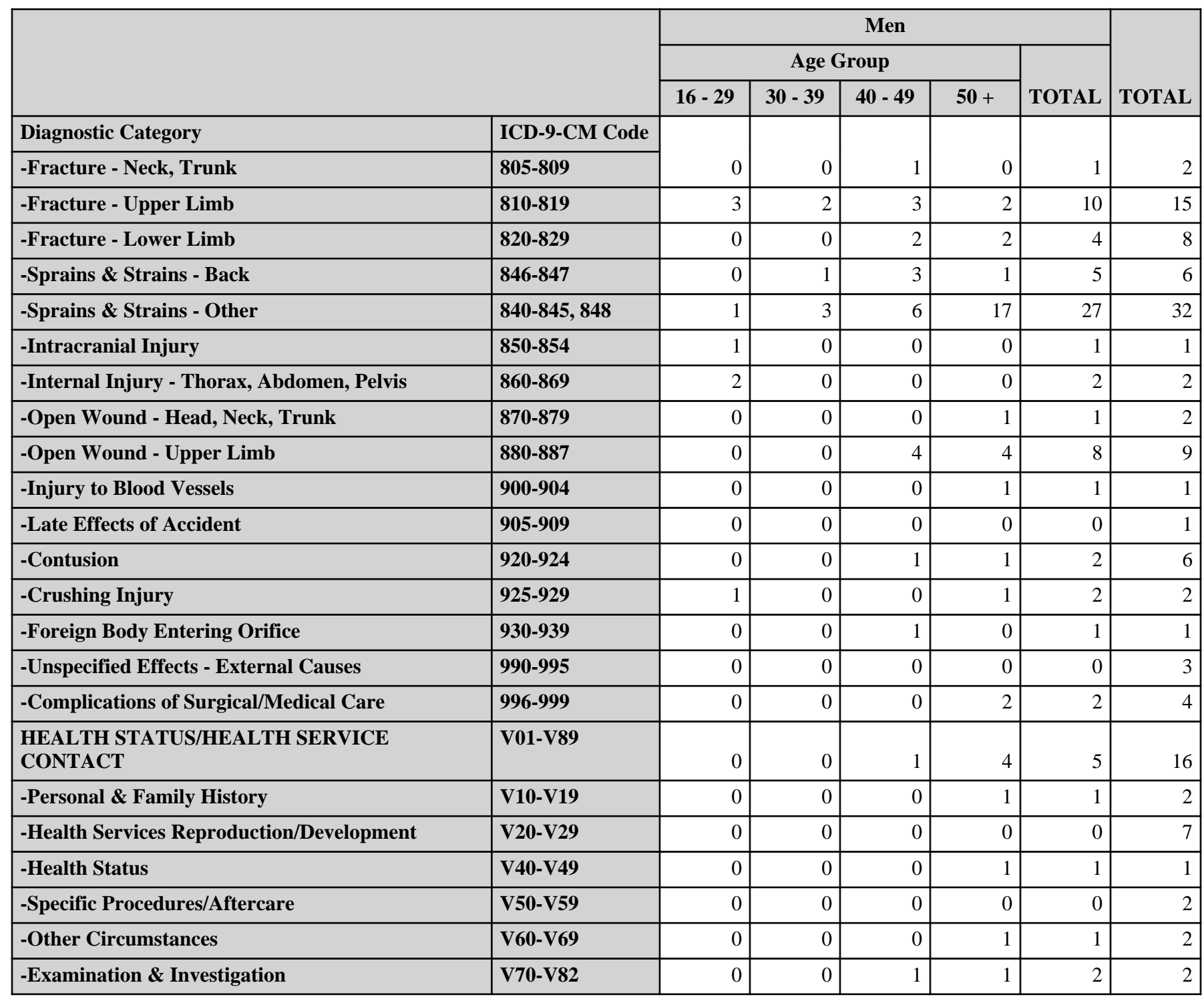

\begin{tabular}{|c|c|c|c|c|c|c|c|c|c|c|c|}
\hline & \multicolumn{5}{|c|}{ Women } & \multicolumn{5}{|c|}{ Men } & \multirow[b]{3}{*}{ TOTAL } \\
\hline & \multicolumn{4}{|c|}{ Age Group } & \multirow[b]{2}{*}{ TOTAL } & \multicolumn{4}{|c|}{ Age Group } & \multirow[b]{2}{*}{ TOTAL } & \\
\hline & $16-29$ & 30 - 39 & $40-49$ & $50+$ & & $16-29$ & $30-39$ & $40-49$ & $50+$ & & \\
\hline Diagnostic Category & \multirow[b]{2}{*}{4} & \multirow[b]{2}{*}{61} & \multirow[b]{2}{*}{61} & \multirow[b]{2}{*}{130} & \multirow[b]{2}{*}{256} & \multirow[b]{2}{*}{18} & \multirow[b]{2}{*}{46} & \multirow[b]{2}{*}{107} & \multirow[b]{2}{*}{243} & \multirow[b]{2}{*}{414} & \multirow[b]{2}{*}{670} \\
\hline Total & & & & & & & & & & & \\
\hline
\end{tabular}

*Only those diagnostic categories and gender/age combinations with at least one occurrence appear in this table. 
Brookhaven National Laboratory 2010

Absence Data

Appendix H. Total Number of Calendar Days Absent in Each Diagnostic Category by Gender and Age*

\begin{tabular}{|c|c|c|c|c|c|c|}
\hline & & \multicolumn{5}{|c|}{ Women } \\
\hline & & \multicolumn{4}{|c|}{ Age Group } & \multirow[b]{2}{*}{ TOTAL } \\
\hline & & $16-29$ & $30-39$ & $40-49$ & $50+$ & \\
\hline Diagnostic Category & ICD-9-CM Code & \multirow[b]{2}{*}{0} & \multirow[b]{2}{*}{0} & \multirow[b]{2}{*}{23} & \multirow[b]{2}{*}{19} & \multirow[b]{2}{*}{42} \\
\hline INFECTIOUS \& PARASITIC DISEASES (DIS) & 001-139 & & & & & \\
\hline MALIGNANT NEOPLASMS & $\begin{array}{l}140-208 \\
209.0-209.3 \\
230-234\end{array}$ & 0 & 0 & 0 & 41 & 41 \\
\hline BENIGN \& UNCERTAIN NEOPLASMS & \begin{tabular}{|l|}
$209.4-209.6$ \\
$210-229,235-239$
\end{tabular} & 0 & 91 & 81 & 12 & 184 \\
\hline ENDOCRINE/METABOLIC/IMMUNITY & $240-279$ & 0 & 7 & 543 & 7 & 557 \\
\hline MENTAL DISORDERS & $290-319$ & 0 & 30 & 8 & 260 & 298 \\
\hline NERVOUS SYSTEM (NS) \& SENSE ORGANS & $320-389$ & 0 & 85 & 17 & 286 & 388 \\
\hline CIRCULATORY SYSTEM & $390-459$ & 0 & 12 & 543 & 20 & 575 \\
\hline RESPIRATORY SYSTEM & $460-519$ & 9 & 37 & 102 & 208 & 356 \\
\hline DIGESTIVE SYSTEM & $520-579$ & 0 & 151 & 79 & 61 & 291 \\
\hline GENITOURINARY SYSTEM & $580-629$ & 0 & 47 & 43 & 37 & 127 \\
\hline SKIN AND SUBCUTANEOUS TISSUE & $680-709$ & 0 & 0 & 0 & 22 & 22 \\
\hline $\begin{array}{l}\text { MUSCULOSKELETAL \& CONNECTIVE } \\
\text { TISSUE }\end{array}$ & $710-739$ & 0 & 527 & 621 & 838 & 1,986 \\
\hline CONGENITAL ANOMALIES & $740-759$ & 0 & 0 & 0 & 53 & 53 \\
\hline $\begin{array}{l}\text { SYMPTOMS, SIGNS, \& ILL-DEFINED } \\
\text { CONDITIONS }\end{array}$ & 780-799 & 0 & 17 & 37 & 41 & 95 \\
\hline INJURY \& POISONING & 800-999 & 0 & 563 & 25 & 504 & 1,092 \\
\hline
\end{tabular}

\footnotetext{
*Absences with >1 ICD-9-CM code in the same diagnostic category were counted only once. Only those diagnostic categories and gender/age combinations with at least one occurrence appear in this table.
} 
Brookhaven National Laboratory 2010

Absence Data

Appendix H. Total Number of Calendar Days Absent in Each Diagnostic Category by Gender and Age*

\begin{tabular}{|c|c|c|c|c|c|c|c|}
\hline & & \multicolumn{5}{|c|}{ Men } & \multirow[b]{3}{*}{ TOTAL } \\
\hline & & \multicolumn{4}{|c|}{ Age Group } & \multirow[b]{2}{*}{ TOTAL } & \\
\hline & & $16-29$ & $30-39$ & $40-49$ & $50+$ & & \\
\hline INFECTIOUS \& PARASITIC DISEASES (DIS) & 001-139 & 2 & 40 & 48 & 33 & 123 & 165 \\
\hline MALIGNANT NEOPLASMS & $\begin{array}{l}140-208, \\
209.0-209.3 \\
230-234\end{array}$ & 0 & 0 & 41 & 565 & 606 & 647 \\
\hline BENIGN \& UNCERTAIN NEOPLASMS & $\begin{array}{l}209.4-209.6 \\
210-229,235-239\end{array}$ & 0 & 0 & 6 & 0 & 6 & 190 \\
\hline ENDOCRINE/METABOLIC/IMMUNITY & $240-279$ & 0 & 0 & 40 & 258 & 298 & 855 \\
\hline MENTAL DISORDERS & $290-319$ & 0 & 0 & 34 & 169 & 203 & 501 \\
\hline DIGESTIVE SYSTEM & $520-579$ & 23 & 119 & 81 & 629 & 852 & 1,143 \\
\hline GENITOURINARY SYSTEM & $580-629$ & 0 & 0 & 28 & 27 & 55 & 182 \\
\hline SKIN AND SUBCUTANEOUS TISSUE & $680-709$ & 0 & 0 & 6 & 116 & 122 & 144 \\
\hline $\begin{array}{l}\text { MUSCULOSKELETAL \& CONNECTIVE } \\
\text { TISSUE }\end{array}$ & $710-739$ & 17 & 56 & 421 & 1,357 & 1,851 & 3,837 \\
\hline CONGENITAL ANOMALIES & $740-759$ & 0 & 0 & 0 & 0 & 0 & 53 \\
\hline $\begin{array}{l}\text { SYMPTOMS, SIGNS, \& ILL-DEFINED } \\
\text { CONDITIONS }\end{array}$ & $780-799$ & 0 & 37 & 44 & 263 & 344 & 439 \\
\hline INJURY \& POISONING & 800-999 & 34 & 177 & 540 & 1,065 & 1,816 & 2,908 \\
\hline
\end{tabular}

\footnotetext{
*Absences with >1 ICD-9-CM code in the same diagnostic category were counted only once. Only those diagnostic categories and gender/age combinations with at least one occurrence appear in this table.
} 


\section{Brookhaven National Laboratory 2010}

Absence Data

Appendix I. Number of Diagnoses in Each Diagnostic Category by Gender and Job Category*

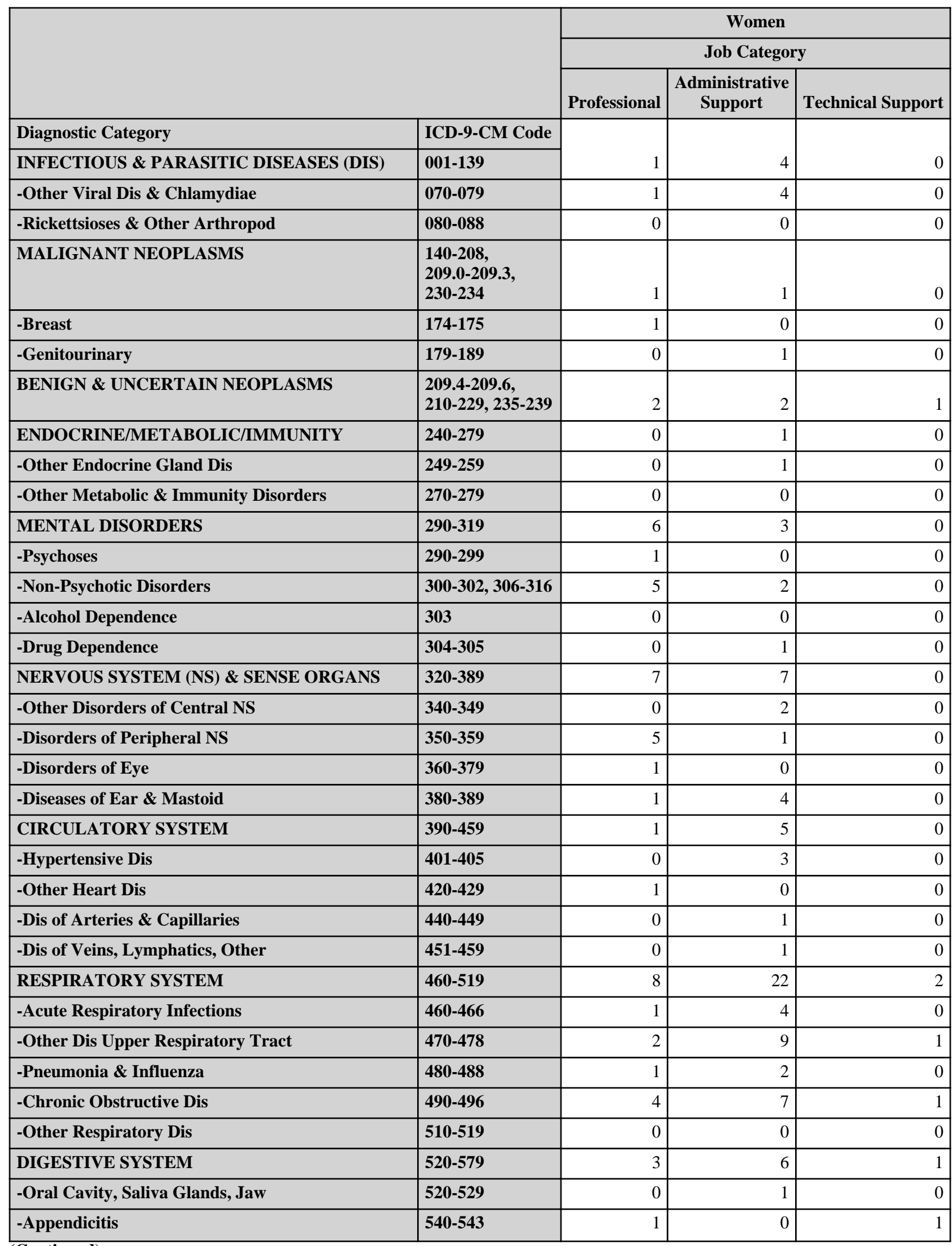

(Continued)

*Only those diagnostic categories and gender/job category combinations with at least one occurrence appear in this table. 
Brookhaven National Laboratory 2010

Absence Data

Appendix I. Number of Diagnoses in Each Diagnostic Category by Gender and Job Category*

\begin{tabular}{|c|c|c|c|c|c|}
\hline & & \multicolumn{4}{|c|}{ Women } \\
\hline & & \multicolumn{3}{|c|}{ Job Category } & \multirow[b]{2}{*}{ TOTAL } \\
\hline & & Service & Security and Fire & Crafts & \\
\hline Diagnostic Category & ICD-9-CM Code & \multirow[b]{2}{*}{0} & \multirow[b]{2}{*}{1} & \multirow[b]{2}{*}{0} & \multirow[b]{2}{*}{6} \\
\hline INFECTIOUS \& PARASITIC DISEASES (DIS) & 001-139 & & & & \\
\hline -Other Viral Dis \& Chlamydiae & 070-079 & 0 & 0 & 0 & 5 \\
\hline -Rickettsioses \& Other Arthropod & 080-088 & 0 & 1 & 0 & 1 \\
\hline MALIGNANT NEOPLASMS & $\begin{array}{l}140-208, \\
209.0-209.3, \\
230-234\end{array}$ & 0 & 0 & 0 & 2 \\
\hline -Breast & \begin{tabular}{|l|}
$174-175$ \\
\end{tabular} & 0 & 0 & 0 & 1 \\
\hline -Genitourinary & 179-189 & 0 & 0 & 0 & 1 \\
\hline BENIGN \& UNCERTAIN NEOPLASMS & $\begin{array}{l}209.4-209.6, \\
210-229,235-239\end{array}$ & 0 & 0 & 0 & 5 \\
\hline ENDOCRINE/METABOLIC/IMMUNITY & $240-279$ & 2 & 0 & 0 & 3 \\
\hline -Other Endocrine Gland Dis & 249-259 & 1 & 0 & 0 & 2 \\
\hline -Other Metabolic \& Immunity Disorders & $270-279$ & 1 & 0 & 0 & 1 \\
\hline MENTAL DISORDERS & $290-319$ & 1 & 0 & 0 & 10 \\
\hline -Psychoses & 290-299 & 0 & 0 & 0 & 1 \\
\hline -Non-Psychotic Disorders & $300-302,306-316$ & 0 & 0 & 0 & 7 \\
\hline -Alcohol Dependence & 303 & 1 & 0 & 0 & 1 \\
\hline -Drug Dependence & $304-305$ & 0 & 0 & 0 & 1 \\
\hline NERVOUS SYSTEM (NS) \& SENSE ORGANS & $320-389$ & 1 & 1 & 1 & 17 \\
\hline -Other Disorders of Central NS & $340-349$ & 0 & 0 & 0 & 2 \\
\hline -Disorders of Peripheral NS & $350-359$ & 0 & 0 & 1 & 7 \\
\hline -Disorders of Eye & $360-379$ & 0 & 0 & 0 & 1 \\
\hline -Diseases of Ear \& Mastoid & $380-389$ & 1 & 1 & 0 & 7 \\
\hline CIRCULATORY SYSTEM & $390-459$ & 1 & 0 & 0 & 7 \\
\hline -Hypertensive Dis & $401-405$ & 0 & 0 & 0 & 3 \\
\hline -Other Heart Dis & $420-429$ & 0 & 0 & 0 & 1 \\
\hline -Dis of Arteries \& Capillaries & $440-449$ & 0 & 0 & 0 & 1 \\
\hline -Dis of Veins, Lymphatics, Other & $451-459$ & 1 & 0 & 0 & 2 \\
\hline RESPIRATORY SYSTEM & $460-519$ & 18 & 5 & 1 & 56 \\
\hline -Acute Respiratory Infections & $460-466$ & 5 & 1 & 0 & 11 \\
\hline -Other Dis Upper Respiratory Tract & $470-478$ & 2 & 3 & 0 & 17 \\
\hline -Pneumonia \& Influenza & $480-488$ & 1 & 1 & 0 & 5 \\
\hline -Chronic Obstructive Dis & $490-496$ & 9 & 0 & 1 & 22 \\
\hline -Other Respiratory Dis & $510-519$ & 1 & 0 & 0 & 1 \\
\hline DIGESTIVE SYSTEM & $520-579$ & 3 & 0 & 0 & 13 \\
\hline -Oral Cavity, Saliva Glands, Jaw & $520-529$ & 0 & 0 & 0 & 1 \\
\hline -Appendicitis & $540-543$ & 0 & 0 & 0 & 2 \\
\hline
\end{tabular}

(Continued)

*Only those diagnostic categories and gender/job category combinations with at least one occurrence appear in this table. 


\section{Brookhaven National Laboratory 2010}

Absence Data

Appendix I. Number of Diagnoses in Each Diagnostic Category by Gender and Job Category*

\begin{tabular}{|c|c|c|c|c|}
\hline & \multicolumn{3}{|c|}{ Women } \\
\hline & & \multicolumn{3}{|c|}{ Job Category } \\
\hline & & Professional & $\begin{array}{c}\text { Administrative } \\
\text { Support }\end{array}$ & Technical Support \\
\hline Diagnostic Category & ICD-9-CM Code & \multirow[b]{2}{*}{0} & \multirow[b]{2}{*}{0} & \multirow[b]{2}{*}{0} \\
\hline -Hernias & $550-553$ & & & \\
\hline -Enteritis, Colitis & $555-558$ & 0 & 3 & 0 \\
\hline -Other Intestinal Dis & $560-569$ & 2 & 0 & 0 \\
\hline -Other Digestive Dis & $570-579$ & 0 & 2 & 0 \\
\hline GENITOURINARY SYSTEM & $580-629$ & 0 & 10 & 1 \\
\hline -Other Urinary Dis & 590-599 & 0 & 2 & 0 \\
\hline -Breast Disorders & 610-612 & 0 & 3 & 0 \\
\hline -Other Female Disorders & $617-629$ & 0 & 5 & 1 \\
\hline SKIN AND SUBCUTANEOUS TISSUE & 680-709 & 0 & 2 & 0 \\
\hline -Infections & $680-686$ & 0 & 1 & 0 \\
\hline -Other Inflammatory Conditions & 690-698 & 0 & 1 & 0 \\
\hline $\begin{array}{l}\text { MUSCULOSKELETAL \& CONNECTIVE } \\
\text { TISSUE }\end{array}$ & 710-739 & 9 & 23 & 1 \\
\hline -Arthropathies & $710-719$ & 3 & 9 & 0 \\
\hline -Dorsopathies & $720-724$ & 3 & 10 & 1 \\
\hline -Rheumatism, Excluding Back & 725-729 & 2 & 2 & 0 \\
\hline -Other Dis \& Acquired Deformities & 730-739 & 1 & 2 & 0 \\
\hline CONGENITAL ANOMALIES & $740-759$ & 1 & 0 & 0 \\
\hline $\begin{array}{l}\text { SYMPTOMS, SIGNS, \& ILL-DEFINED } \\
\text { CONDITIONS }\end{array}$ & 780-799 & 8 & 18 & 0 \\
\hline -Symptoms & 780-789 & 8 & 17 & 0 \\
\hline -Non-Specific Abnormal Findings & $790-796$ & 0 & 1 & 0 \\
\hline INJURY \& POISONING & $800-999$ & 17 & 4 & 2 \\
\hline -Fracture - Neck, Trunk & $805-809$ & 0 & 1 & 0 \\
\hline -Fracture - Upper Limb & $810-819$ & 2 & 0 & 1 \\
\hline -Fracture - Lower Limb & 820-829 & 3 & 1 & 0 \\
\hline -Sprains \& Strains - Back & 846-847 & 0 & 0 & 0 \\
\hline -Sprains \& Strains - Other & $840-845,848$ & 4 & 0 & 0 \\
\hline -Open Wound - Head, Neck, Trunk & 870-879 & 0 & 0 & 0 \\
\hline -Open Wound - Upper Limb & 880-887 & 1 & 0 & 0 \\
\hline -Late Effects of Accident & 905-909 & 1 & 0 & 0 \\
\hline -Contusion & 920-924 & 4 & 0 & 0 \\
\hline -Unspecified Effects - External Causes & $990-995$ & 1 & 1 & 1 \\
\hline -Complications of Surgical/Medical Care & 996-999 & 1 & 1 & 0 \\
\hline $\begin{array}{l}\text { HEALTH STATUS/HEALTH SERVICE } \\
\text { CONTACT }\end{array}$ & V01-V89 & 5 & 5 & 0 \\
\hline -Personal \& Family History & V10-V19 & 0 & 0 & 0 \\
\hline
\end{tabular}

(Continued)

*Only those diagnostic categories and gender/job category combinations with at least one occurrence appear in this table. 


\section{Brookhaven National Laboratory 2010}

Absence Data

Appendix I. Number of Diagnoses in Each Diagnostic Category by Gender and Job Category*

\begin{tabular}{|c|c|c|c|c|c|}
\hline & & \multicolumn{4}{|c|}{ Women } \\
\hline & & \multicolumn{3}{|c|}{ Job Category } & \multirow[b]{2}{*}{ TOTAL } \\
\hline & & Service & Security and Fire & Crafts & \\
\hline Diagnostic Category & ICD-9-CM Code & & & & \\
\hline -Hernias & $550-553$ & 1 & 0 & 0 & 1 \\
\hline -Enteritis, Colitis & \begin{tabular}{|l|}
$555-558$ \\
\end{tabular} & 1 & 0 & 0 & $\overline{4}$ \\
\hline -Other Intestinal Dis & $560-569$ & 1 & 0 & 0 & 3 \\
\hline -Other Digestive Dis & \begin{tabular}{|l|}
$570-579$ \\
\end{tabular} & 0 & 0 & 0 & 2 \\
\hline GENITOURINARY SYSTEM & $580-629$ & 2 & 0 & 0 & 13 \\
\hline -Other Urinary Dis & $590-599$ & 1 & 0 & 0 & 3 \\
\hline -Breast Disorders & $610-612$ & 1 & 0 & 0 & 4 \\
\hline -Other Female Disorders & $617-629$ & 0 & 0 & 0 & $\overline{6}$ \\
\hline SKIN AND SUBCUTANEOUS TISSUE & 680-709 & 0 & 0 & 0 & 2 \\
\hline -Infections & $680-686$ & 0 & 0 & 0 & 1 \\
\hline -Other Inflammatory Conditions & $690-698$ & 0 & 0 & 0 & 1 \\
\hline $\begin{array}{l}\text { MUSCULOSKELETAL \& CONNECTIVE } \\
\text { TISSUE }\end{array}$ & $710-739$ & 17 & 0 & 2 & 52 \\
\hline -Arthropathies & $710-719$ & 7 & 0 & 2 & 21 \\
\hline -Dorsopathies & $720-724$ & 6 & 0 & 0 & 20 \\
\hline -Rheumatism, Excluding Back & $725-729$ & 4 & 0 & 0 & 8 \\
\hline -Other Dis \& Acquired Deformities & 730-739 & 0 & 0 & 0 & 3 \\
\hline CONGENITAL ANOMALIES & \begin{tabular}{|l|}
$740-759$ \\
\end{tabular} & 0 & 0 & 0 & 1 \\
\hline $\begin{array}{l}\text { SYMPTOMS, SIGNS, \& ILL-DEFINED } \\
\text { CONDITIONS }\end{array}$ & 780-799 & 4 & 0 & 0 & 30 \\
\hline -Symptoms & 780-789 & 4 & 0 & 0 & 29 \\
\hline -Non-Specific Abnormal Findings & $790-796$ & 0 & 0 & 0 & 1 \\
\hline INJURY \& POISONING & $800-999$ & 5 & 0 & 0 & 28 \\
\hline -Fracture - Neck, Trunk & 805-809 & 0 & 0 & 0 & 1 \\
\hline -Fracture - Upper Limb & $810-819$ & 2 & 0 & 0 & 5 \\
\hline -Fracture - Lower Limb & $820-829$ & 0 & 0 & 0 & 4 \\
\hline -Sprains \& Strains - Back & 846-847 & 1 & 0 & 0 & 1 \\
\hline -Sprains \& Strains - Other & $840-845,848$ & 1 & 0 & 0 & 5 \\
\hline -Open Wound - Head, Neck, Trunk & $870-879$ & 1 & 0 & 0 & 1 \\
\hline -Open Wound - Upper Limb & $880-887$ & 0 & 0 & 0 & 1 \\
\hline -Late Effects of Accident & 905-909 & 0 & 0 & 0 & 1 \\
\hline -Contusion & $920-924$ & 0 & 0 & 0 & 4 \\
\hline -Unspecified Effects - External Causes & $990-995$ & 0 & 0 & 0 & 3 \\
\hline -Complications of Surgical/Medical Care & 996-999 & 0 & 0 & 0 & 2 \\
\hline $\begin{array}{l}\text { HEALTH STATUS/HEALTH SERVICE } \\
\text { CONTACT }\end{array}$ & V01-V89 & 1 & 0 & 0 & 11 \\
\hline -Personal \& Family History & \begin{tabular}{|l|} 
V10-V19 \\
\end{tabular} & 1 & 0 & 0 & 1 \\
\hline
\end{tabular}

(Continued)

*Only those diagnostic categories and gender/job category combinations with at least one occurrence appear in this table. 
Brookhaven National Laboratory 2010

Absence Data

Appendix I. Number of Diagnoses in Each Diagnostic Category by Gender and Job Category*

\begin{tabular}{|c|c|c|c|c|}
\hline & & \multicolumn{3}{|c|}{ Women } \\
\hline & & \multicolumn{3}{|c|}{ Job Category } \\
\hline & & Professional & $\begin{array}{l}\text { Administrative } \\
\text { Support }\end{array}$ & Technical Support \\
\hline Diagnostic Category & ICD-9-CM Code & \multirow[b]{2}{*}{4} & \multirow[b]{2}{*}{3} & \multirow[b]{2}{*}{0} \\
\hline -Health Services Reproduction/Development & V20-V29 & & & \\
\hline -Specific Procedures/Aftercare & V50-V59 & 1 & 1 & $\overline{0}$ \\
\hline -Other Circumstances & V60-V69 & 0 & 1 & 0 \\
\hline
\end{tabular}

*Only those diagnostic categories and gender/job category combinations with at least one occurrence appear in this table. 
Brookhaven National Laboratory 2010

Absence Data

Appendix I. Number of Diagnoses in Each Diagnostic Category by Gender and Job Category*

\begin{tabular}{|c|c|c|c|c|c|}
\hline & & \multicolumn{4}{|c|}{ Women } \\
\hline & & \multicolumn{3}{|c|}{ Job Category } & \multirow[b]{2}{*}{ TOTAL } \\
\hline & & Service & Security and Fire & Crafts & \\
\hline Diagnostic Category & ICD-9-CM Code & \multirow[b]{2}{*}{0} & \multirow[b]{2}{*}{0} & \multirow[b]{2}{*}{0} & \multirow[b]{2}{*}{7} \\
\hline -Health Services Reproduction/Development & V20-V29 & & & & \\
\hline -Specific Procedures/Aftercare & V50-V59 & 0 & 0 & 0 & 2 \\
\hline -Other Circumstances & V60-V69 & 0 & 0 & 0 & 1 \\
\hline
\end{tabular}

\begin{tabular}{|c|c|c|c|c|c|c|c|}
\hline & \multicolumn{7}{|c|}{ Women } \\
\hline & \multicolumn{6}{|c|}{ Job Category } & \multirow[b]{2}{*}{ TOTAL } \\
\hline & Professional & $\begin{array}{c}\text { Administrative } \\
\text { Support }\end{array}$ & Technical Support & Service & Security and Fire & Crafts & \\
\hline Diagnostic Category & \multirow[b]{2}{*}{69} & \multirow[b]{2}{*}{113} & \multirow[b]{2}{*}{8} & \multirow[b]{2}{*}{55} & \multirow[b]{2}{*}{7} & \multirow[b]{2}{*}{4} & \multirow[b]{2}{*}{256} \\
\hline Total & & & & & & & \\
\hline
\end{tabular}

*Only those diagnostic categories and gender/job category combinations with at least one occurrence appear in this table. 


\section{Brookhaven National Laboratory 2010}

Absence Data

Appendix I. Number of Diagnoses in Each Diagnostic Category by Gender and Job Category*

\begin{tabular}{|c|c|c|c|c|}
\hline & \multicolumn{3}{|c|}{ Men } \\
\hline & & \multicolumn{3}{|c|}{ Job Category } \\
\hline & & Professional & $\begin{array}{c}\text { Administrative } \\
\text { Support }\end{array}$ & Technical Support \\
\hline Diagnostic Category & ICD-9-CM Code & \multirow[b]{2}{*}{7} & \multirow[b]{2}{*}{0} & \multirow[b]{2}{*}{1} \\
\hline INFECTIOUS \& PARASITIC DISEASES (DIS) & 001-139 & & & \\
\hline -Other Bacterial Dis & $030-041$ & 3 & 0 & 0 \\
\hline -Viral Dis with Exanthem & 050-059 & 0 & 0 & 0 \\
\hline -Other Viral Dis \& Chlamydiae & 070-079 & 3 & 0 & 0 \\
\hline -Rickettsioses \& Other Arthropod & $080-088$ & 1 & 0 & 1 \\
\hline -Other Infections \& Parasitic Dis & $130-136$ & 0 & 0 & 0 \\
\hline MALIGNANT NEOPLASMS & $\begin{array}{l}140-208 \\
209.0-209.3 \\
230-234\end{array}$ & 6 & 0 & 3 \\
\hline -Lip, Oral Cavity, Pharynx & $140-149$ & 0 & 0 & 1 \\
\hline -Digestive \& Peritoneal & 150-159 & 2 & 0 & 0 \\
\hline -Respiratory \& Intrathoracic & $160-165$ & 0 & 0 & $\overline{0}$ \\
\hline -Bone, Connective Tissue, Skin & $170-173,176$ & 1 & 0 & 0 \\
\hline -Genitourinary & 179-189 & 2 & 0 & 1 \\
\hline -Other \& Unspecified Sites & 195-199, 209.7 & 1 & 0 & 0 \\
\hline -Lymphatic \& Hematopoietic & $200-208$ & 0 & 0 & 1 \\
\hline BENIGN \& UNCERTAIN NEOPLASMS & $\begin{array}{l}209.4-209.6 \\
210-229,235-239\end{array}$ & 1 & 0 & 0 \\
\hline ENDOCRINE/METABOLIC/IMMUNITY & $240-279$ & 5 & 0 & 3 \\
\hline -Thyroid Gland Disorders & $240-246$ & 1 & 0 & 0 \\
\hline -Other Endocrine Gland Dis & 249-259 & 2 & 0 & 3 \\
\hline -Other Metabolic \& Immunity Disorders & $270-279$ & 2 & 0 & 0 \\
\hline MENTAL DISORDERS & $290-319$ & 1 & 0 & 2 \\
\hline -Psychoses & 290-299 & 0 & 0 & 1 \\
\hline -Non-Psychotic Disorders & $300-302,306-316$ & 1 & 0 & 1 \\
\hline NERVOUS SYSTEM (NS) \& SENSE ORGANS & 320-389 & 4 & 0 & 5 \\
\hline -Hereditary/Degenerative Central NS Dis & 330-337 & 0 & 0 & 0 \\
\hline -Other Disorders of Central NS & $340-349$ & 1 & 0 & 0 \\
\hline -Disorders of Peripheral NS & $350-359$ & 0 & 0 & 2 \\
\hline -Disorders of Eye & $360-379$ & 2 & 0 & 3 \\
\hline -Diseases of Ear \& Mastoid & $380-389$ & 1 & 0 & 0 \\
\hline CIRCULATORY SYSTEM & $390-459$ & 5 & 1 & 10 \\
\hline -Hypertensive Dis & $401-405$ & 1 & 0 & 4 \\
\hline -Ischemic Heart Dis & $410-414$ & 2 & 1 & 4 \\
\hline -Other Heart Dis & $420-429$ & 2 & 0 & 2 \\
\hline -Dis of Veins, Lymphatics, Other & 451-459 & 0 & 0 & 0 \\
\hline RESPIRATORY SYSTEM & $460-519$ & 10 & 3 & 14 \\
\hline
\end{tabular}

(Continued)

*Only those diagnostic categories and gender/job category combinations with at least one occurrence appear in this table. 


\section{Brookhaven National Laboratory 2010}

Absence Data

Appendix I. Number of Diagnoses in Each Diagnostic Category by Gender and Job Category*

\begin{tabular}{|c|c|c|c|c|c|}
\hline & & \multicolumn{4}{|c|}{ Men } \\
\hline & & \multicolumn{3}{|c|}{ Job Category } & \multirow[b]{2}{*}{ TOTAL } \\
\hline & & Service & Security and Fire & Crafts & \\
\hline Diagnostic Category & ICD-9-CM Code & \multirow[b]{2}{*}{3} & \multirow[b]{2}{*}{1} & \multirow[b]{2}{*}{4} & \multirow[b]{2}{*}{16} \\
\hline INFECTIOUS \& PARASITIC DISEASES (DIS) & 001-139 & & & & \\
\hline -Other Bacterial Dis & $030-041$ & 1 & 0 & 2 & $\overline{6}$ \\
\hline -Viral Dis with Exanthem & 050-059 & 0 & 0 & 1 & 1 \\
\hline -Other Viral Dis \& Chlamydiae & 070-079 & 2 & 0 & 0 & 5 \\
\hline -Rickettsioses \& Other Arthropod & 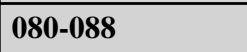 & 0 & 0 & 1 & 3 \\
\hline -Other Infections \& Parasitic Dis & $130-136$ & 0 & 1 & 0 & 1 \\
\hline MALIGNANT NEOPLASMS & $\begin{array}{l}140-208, \\
209.0-209.3 \\
230-234\end{array}$ & 3 & 2 & 0 & 14 \\
\hline -Lip, Oral Cavity, Pharynx & $140-149$ & 0 & 0 & 0 & 1 \\
\hline -Digestive \& Peritoneal & 150-159 & 0 & 0 & 0 & 2 \\
\hline -Respiratory \& Intrathoracic & $160-165$ & 2 & 0 & 0 & 2 \\
\hline -Bone, Connective Tissue, Skin & 170-173, 176 & 1 & 1 & 0 & 3 \\
\hline -Genitourinary & 179-189 & 0 & 1 & 0 & 4 \\
\hline -Other \& Unspecified Sites & 195-199, 209.7 & 0 & 0 & 0 & 1 \\
\hline -Lymphatic \& Hematopoietic & $200-208$ & 0 & 0 & 0 & 1 \\
\hline BENIGN \& UNCERTAIN NEOPLASMS & $\begin{array}{l}209.4-209.6, \\
210-229,235-239\end{array}$ & 0 & 0 & 0 & 1 \\
\hline ENDOCRINE/METABOLIC/IMMUNITY & $240-279$ & 3 & 0 & 1 & 12 \\
\hline -Thyroid Gland Disorders & $240-246$ & 0 & 0 & 0 & 1 \\
\hline -Other Endocrine Gland Dis & $249-259$ & 2 & 0 & 0 & 7 \\
\hline -Other Metabolic \& Immunity Disorders & $270-279$ & 1 & 0 & 1 & 4 \\
\hline MENTAL DISORDERS & $290-319$ & 3 & 1 & 0 & 7 \\
\hline -Psychoses & 290-299 & 0 & 0 & 0 & 1 \\
\hline -Non-Psychotic Disorders & $300-302,306-316$ & 3 & 1 & 0 & 6 \\
\hline NERVOUS SYSTEM (NS) \& SENSE ORGANS & $320-389$ & 13 & 2 & 3 & 27 \\
\hline -Hereditary/Degenerative Central NS Dis & 330-337 & 0 & 1 & 0 & 1 \\
\hline -Other Disorders of Central NS & $340-349$ & 1 & 0 & 0 & 2 \\
\hline -Disorders of Peripheral NS & $350-359$ & 4 & 0 & 1 & 7 \\
\hline -Disorders of Eye & $360-379$ & 6 & 1 & 1 & 13 \\
\hline -Diseases of Ear \& Mastoid & $380-389$ & 2 & 0 & 1 & 4 \\
\hline CIRCULATORY SYSTEM & $390-459$ & 9 & 3 & 1 & 29 \\
\hline -Hypertensive Dis & $401-405$ & 1 & 0 & 0 & 6 \\
\hline -Ischemic Heart Dis & $410-414$ & 2 & 1 & 0 & 10 \\
\hline -Other Heart Dis & $420-429$ & 4 & 2 & 1 & 11 \\
\hline -Dis of Veins, Lymphatics, Other & $451-459$ & 2 & 0 & 0 & 2 \\
\hline RESPIRATORY SYSTEM & $460-519$ & 12 & 2 & 14 & 55 \\
\hline
\end{tabular}

(Continued)

*Only those diagnostic categories and gender/job category combinations with at least one occurrence appear in this table. 


\section{Brookhaven National Laboratory 2010}

Absence Data

Appendix I. Number of Diagnoses in Each Diagnostic Category by Gender and Job Category*

\begin{tabular}{|c|c|c|c|c|}
\hline & \multicolumn{3}{|c|}{ Men } \\
\hline & & \multicolumn{3}{|c|}{ Job Category } \\
\hline & & Professional & $\begin{array}{l}\text { Administrative } \\
\text { Support }\end{array}$ & Technical Support \\
\hline Diagnostic Category & ICD-9-CM Code & \multirow[b]{2}{*}{6} & \multirow[b]{2}{*}{2} & \multirow[b]{2}{*}{6} \\
\hline -Acute Respiratory Infections & $460-466$ & & & \\
\hline -Other Dis Upper Respiratory Tract & $470-478$ & 0 & 1 & 2 \\
\hline -Pneumonia \& Influenza & $480-488$ & 1 & 0 & 2 \\
\hline -Chronic Obstructive Dis & $490-496$ & 1 & 0 & 4 \\
\hline -Other Respiratory Dis & $510-519$ & 2 & 0 & $\overline{0}$ \\
\hline DIGESTIVE SYSTEM & $520-579$ & 15 & 2 & 7 \\
\hline -Oral Cavity, Saliva Glands, Jaw & $520-529$ & 1 & 0 & 1 \\
\hline -Esophagus, Stomach, Duodenum & $530-538$ & 0 & 0 & 0 \\
\hline -Appendicitis & $540-543$ & 1 & 0 & 1 \\
\hline -Hernias & $550-553$ & 5 & 0 & 4 \\
\hline -Enteritis, Colitis & $555-558$ & 2 & 1 & 1 \\
\hline -Other Intestinal Dis & $560-569$ & 3 & 0 & 0 \\
\hline -Other Digestive Dis & $570-579$ & 3 & 1 & 0 \\
\hline GENITOURINARY SYSTEM & $580-629$ & 0 & 0 & 5 \\
\hline -Other Urinary Dis & $590-599$ & 0 & 0 & 3 \\
\hline -Male Genital Organ Dis & $600-608$ & 0 & 0 & 2 \\
\hline SKIN AND SUBCUTANEOUS TISSUE & 680-709 & 1 & 1 & 2 \\
\hline -Infections & $680-686$ & 1 & 0 & 1 \\
\hline -Other Inflammatory Conditions & $690-698$ & 0 & 1 & 0 \\
\hline -Other & 700-709 & 0 & 0 & 1 \\
\hline $\begin{array}{l}\text { MUSCULOSKELETAL \& CONNECTIVE } \\
\text { TISSUE }\end{array}$ & 710-739 & 13 & 0 & 16 \\
\hline -Arthropathies & 710-719 & 5 & 0 & 5 \\
\hline -Dorsopathies & $720-724$ & 7 & 0 & 8 \\
\hline -Rheumatism, Excluding Back & 725-729 & 1 & 0 & 3 \\
\hline -Other Dis \& Acquired Deformities & $730-739$ & 0 & 0 & 0 \\
\hline $\begin{array}{l}\text { SYMPTOMS, SIGNS, \& ILL-DEFINED } \\
\text { CONDITIONS }\end{array}$ & $780-799$ & 5 & 0 & 12 \\
\hline -Symptoms & 780-789 & 4 & 0 & 12 \\
\hline -Non-Specific Abnormal Findings & $790-796$ & 1 & 0 & 0 \\
\hline INJURY \& POISONING & 800-999 & 11 & 1 & 16 \\
\hline -Fracture - Neck, Trunk & 805-809 & 1 & 0 & 0 \\
\hline -Fracture - Upper Limb & 810-819 & 3 & 0 & 3 \\
\hline -Fracture - Lower Limb & $820-829$ & 2 & 0 & 1 \\
\hline -Sprains \& Strains - Back & 846-847 & 1 & 0 & 0 \\
\hline -Sprains \& Strains - Other & $840-845,848$ & 3 & 0 & 6 \\
\hline -Intracranial Injury & $850-854$ & 0 & 1 & 0 \\
\hline
\end{tabular}

(Continued)

*Only those diagnostic categories and gender/job category combinations with at least one occurrence appear in this table. 


\section{Brookhaven National Laboratory 2010}

Absence Data

Appendix I. Number of Diagnoses in Each Diagnostic Category by Gender and Job Category*

\begin{tabular}{|c|c|c|c|c|c|}
\hline & & \multicolumn{4}{|c|}{ Men } \\
\hline & & \multicolumn{3}{|c|}{ Job Category } & \multirow[b]{2}{*}{ TOTAL } \\
\hline & & Service & Security and Fire & Crafts & \\
\hline Diagnostic Category & ICD-9-CM Code & \multirow[b]{2}{*}{6} & \multirow[b]{2}{*}{1} & \multirow[b]{2}{*}{7} & \multirow[b]{2}{*}{28} \\
\hline -Acute Respiratory Infections & $460-466$ & & & & \\
\hline -Other Dis Upper Respiratory Tract & $470-478$ & 4 & 1 & 1 & 9 \\
\hline -Pneumonia \& Influenza & $480-488$ & 0 & 0 & 1 & 4 \\
\hline -Chronic Obstructive Dis & $490-496$ & 2 & 0 & 5 & 12 \\
\hline -Other Respiratory Dis & $510-519$ & 0 & 0 & 0 & 2 \\
\hline DIGESTIVE SYSTEM & $520-579$ & 15 & 1 & 12 & 52 \\
\hline -Oral Cavity, Saliva Glands, Jaw & $520-529$ & 0 & 0 & 1 & 3 \\
\hline -Esophagus, Stomach, Duodenum & \begin{tabular}{|l|}
$530-538$ \\
\end{tabular} & 1 & 0 & 0 & 1 \\
\hline -Appendicitis & $540-543$ & 0 & 1 & 0 & 3 \\
\hline -Hernias & $550-553$ & 3 & 0 & 1 & 13 \\
\hline -Enteritis, Colitis & $555-558$ & 8 & 0 & 6 & 18 \\
\hline -Other Intestinal Dis & $560-569$ & 2 & 0 & 4 & 9 \\
\hline -Other Digestive Dis & $570-579$ & 1 & 0 & 0 & 5 \\
\hline GENITOURINARY SYSTEM & $580-629$ & 0 & 1 & 3 & 9 \\
\hline -Other Urinary Dis & $590-599$ & 0 & 1 & 3 & 7 \\
\hline -Male Genital Organ Dis & $600-608$ & 0 & 0 & 0 & 2 \\
\hline SKIN AND SUBCUTANEOUS TISSUE & $680-709$ & 2 & 0 & 1 & 7 \\
\hline -Infections & $680-686$ & 0 & 0 & 0 & 2 \\
\hline -Other Inflammatory Conditions & $690-698$ & 0 & 0 & 1 & 2 \\
\hline -Other & 700-709 & 2 & 0 & 0 & 3 \\
\hline $\begin{array}{l}\text { MUSCULOSKELETAL \& CONNECTIVE } \\
\text { TISSUE }\end{array}$ & 710-739 & 29 & 6 & 16 & 80 \\
\hline -Arthropathies & $710-719$ & 16 & 2 & 5 & 33 \\
\hline -Dorsopathies & $720-724$ & 11 & 4 & 6 & 36 \\
\hline -Rheumatism, Excluding Back & 725-729 & 2 & 0 & 4 & 10 \\
\hline -Other Dis \& Acquired Deformities & $730-739$ & 0 & 0 & 1 & 1 \\
\hline $\begin{array}{l}\text { SYMPTOMS, SIGNS, \& ILL-DEFINED } \\
\text { CONDITIONS }\end{array}$ & 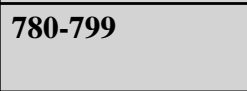 & 9 & 1 & 6 & 33 \\
\hline -Symptoms & 780-789 & 9 & 1 & 4 & 30 \\
\hline -Non-Specific Abnormal Findings & $790-796$ & 0 & 0 & 2 & 3 \\
\hline INJURY \& POISONING & $800-999$ & 17 & 3 & 19 & 67 \\
\hline -Fracture - Neck, Trunk & 805-809 & 0 & 0 & 0 & 1 \\
\hline -Fracture - Upper Limb & 810-819 & 2 & 0 & 2 & 10 \\
\hline -Fracture - Lower Limb & $820-829$ & 0 & 0 & 1 & 4 \\
\hline -Sprains \& Strains - Back & 846-847 & 2 & 0 & 2 & 5 \\
\hline -Sprains \& Strains - Other & $840-845,848$ & 8 & 1 & 9 & 27 \\
\hline -Intracranial Injury & $850-854$ & 0 & 0 & 0 & 1 \\
\hline
\end{tabular}

(Continued)

*Only those diagnostic categories and gender/job category combinations with at least one occurrence appear in this table. 
Brookhaven National Laboratory 2010

Absence Data

Appendix I. Number of Diagnoses in Each Diagnostic Category by Gender and Job Category*

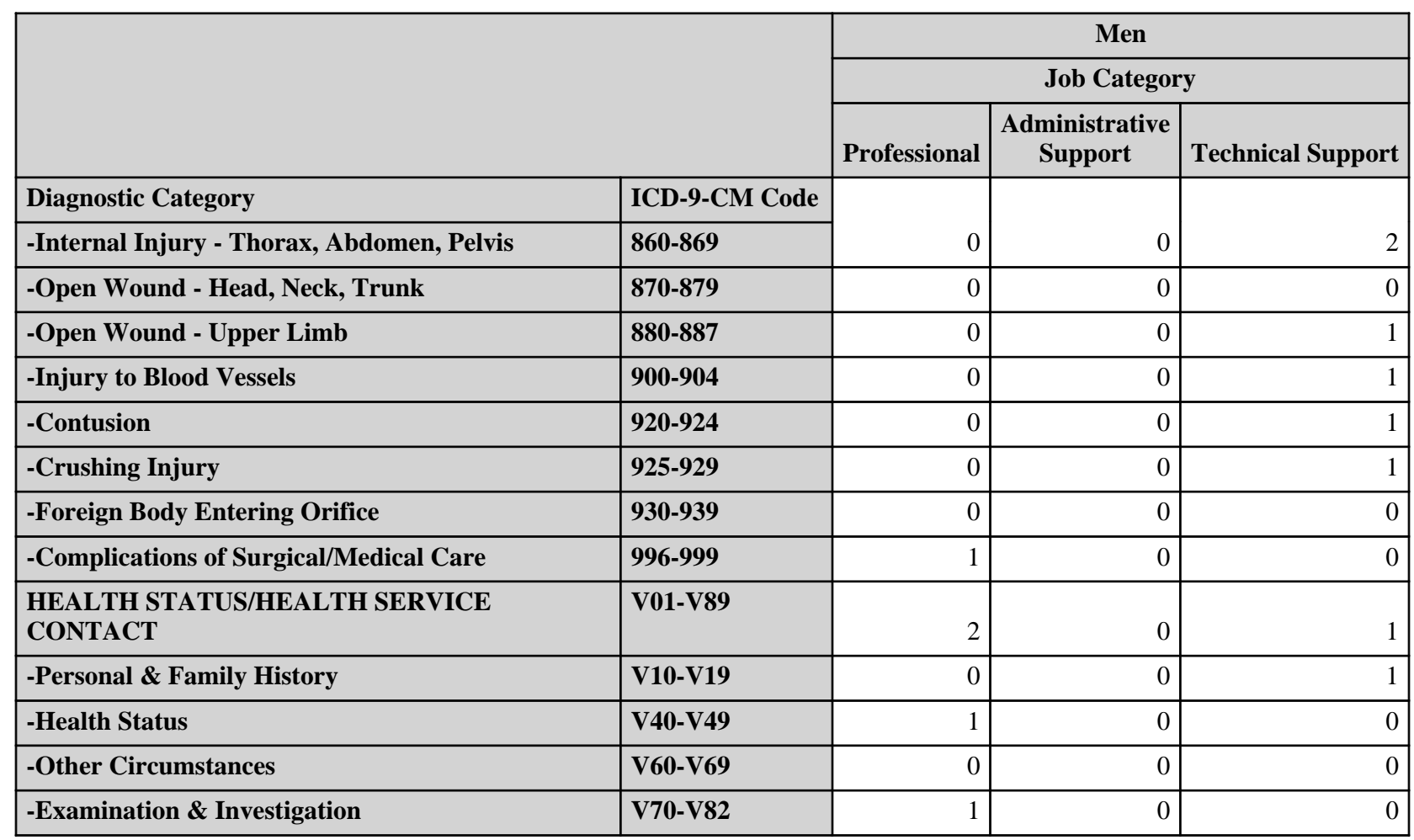

*Only those diagnostic categories and gender/job category combinations with at least one occurrence appear in this table. 
Brookhaven National Laboratory 2010

Absence Data

Appendix I. Number of Diagnoses in Each Diagnostic Category by Gender and Job Category*

\begin{tabular}{|c|c|c|c|c|c|}
\hline & & \multicolumn{4}{|c|}{ Men } \\
\hline & & \multicolumn{3}{|c|}{ Job Category } & \multirow[b]{2}{*}{ TOTAL } \\
\hline & & Service & Security and Fire & Crafts & \\
\hline Diagnostic Category & ICD-9-CM Code & \multirow[b]{2}{*}{0} & \multirow[b]{2}{*}{0} & \multirow[b]{2}{*}{0} & \multirow[b]{2}{*}{2} \\
\hline -Internal Injury - Thorax, Abdomen, Pelvis & $860-869$ & & & & \\
\hline -Open Wound - Head, Neck, Trunk & 870-879 & 0 & 0 & 1 & 1 \\
\hline -Open Wound - Upper Limb & $880-887$ & 4 & 1 & 2 & 8 \\
\hline -Injury to Blood Vessels & 900-904 & 0 & 0 & 0 & 1 \\
\hline -Contusion & 920-924 & 0 & 0 & 1 & 2 \\
\hline -Crushing Injury & 925-929 & 0 & 0 & 1 & 2 \\
\hline -Foreign Body Entering Orifice & 930-939 & 0 & 1 & 0 & 1 \\
\hline -Complications of Surgical/Medical Care & 996-999 & 1 & 0 & 0 & 2 \\
\hline $\begin{array}{l}\text { HEALTH STATUS/HEALTH SERVICE } \\
\text { CONTACT }\end{array}$ & V01-V89 & 1 & 0 & 1 & 5 \\
\hline -Personal \& Family History & V10-V19 & 0 & 0 & 0 & 1 \\
\hline -Health Status & V40-V49 & 0 & 0 & 0 & 1 \\
\hline -Other Circumstances & V60-V69 & 1 & 0 & 0 & 1 \\
\hline -Examination \& Investigation & V70-V82 & 0 & 0 & 1 & 2 \\
\hline
\end{tabular}

\begin{tabular}{|c|c|c|c|c|c|c|c|}
\hline & \multicolumn{7}{|c|}{ Men } \\
\hline & \multicolumn{6}{|c|}{ Job Category } & \multirow[b]{2}{*}{ TOTAL } \\
\hline & Professional & \begin{tabular}{|c|}
$\begin{array}{c}\text { Administrative } \\
\text { Support }\end{array}$ \\
\end{tabular} & Technical Support & Service & Security and Fire & Crafts & \\
\hline Diagnostic Category & \multirow[b]{2}{*}{86} & \multirow[b]{2}{*}{8} & \multirow[b]{2}{*}{97} & \multirow[b]{2}{*}{119} & \multirow[b]{2}{*}{23} & \multirow[b]{2}{*}{81} & \multirow[b]{2}{*}{414} \\
\hline Total & & & & & & & \\
\hline
\end{tabular}

*Only those diagnostic categories and gender/job category combinations with at least one occurrence appear in this table. 
Brookhaven National Laboratory 2010

Absence Data

Appendix J. Total Number of Calendar Days Absent in Each Diagnostic Category by Gender and Job Category*

\begin{tabular}{|c|c|c|c|c|}
\hline & & \multicolumn{3}{|c|}{ Women } \\
\hline & & \multicolumn{3}{|c|}{ Job Category } \\
\hline & & Professional & $\begin{array}{l}\text { Administrative } \\
\text { Support }\end{array}$ & Technical Support \\
\hline Diagnostic Category & ICD-9-CM Code & \multirow[b]{2}{*}{13} & \multirow[b]{2}{*}{23} & \multirow[b]{2}{*}{0} \\
\hline INFECTIOUS \& PARASITIC DISEASES (DIS) & 001-139 & & & \\
\hline MALIGNANT NEOPLASMS & $\begin{array}{l}140-208 \\
209.0-209.3 \\
230-234\end{array}$ & 7 & 34 & 0 \\
\hline BENIGN \& UNCERTAIN NEOPLASMS & $\begin{array}{l}209.4-209.6 \\
210-229,235-239\end{array}$ & 132 & 31 & 21 \\
\hline ENDOCRINE/METABOLIC/IMMUNITY & $240-279$ & 0 & 7 & 0 \\
\hline MENTAL DISORDERS & $290-319$ & 209 & 18 & 0 \\
\hline NERVOUS SYSTEM (NS) \& SENSE ORGANS & 320-389 & 329 & 36 & 0 \\
\hline CIRCULATORY SYSTEM & $390-459$ & 5 & 27 & 0 \\
\hline RESPIRATORY SYSTEM & $460-519$ & 75 & 111 & 12 \\
\hline DIGESTIVE SYSTEM & $520-579$ & 108 & 94 & 18 \\
\hline GENITOURINARY SYSTEM & $580-629$ & 0 & 98 & 18 \\
\hline SKIN AND SUBCUTANEOUS TISSUE & $680-709$ & 0 & 22 & 0 \\
\hline $\begin{array}{l}\text { MUSCULOSKELETAL \& CONNECTIVE } \\
\text { TISSUE }\end{array}$ & $710-739$ & 417 & 419 & 5 \\
\hline CONGENITAL ANOMALIES & $740-759$ & 53 & 0 & 0 \\
\hline $\begin{array}{l}\text { SYMPTOMS, SIGNS, \& ILL-DEFINED } \\
\text { CONDITIONS }\end{array}$ & 780-799 & 15 & 55 & 0 \\
\hline INJURY \& POISONING & $800-999$ & 335 & 171 & 22 \\
\hline
\end{tabular}

\footnotetext{
*Absences with >1 ICD-9-CM code in the same diagnostic category were counted only once. Only those diagnostic categories and gender/job category combinations with at least one occurrence appear in this table.
} 
Brookhaven National Laboratory 2010

Absence Data

Appendix J. Total Number of Calendar Days Absent in Each Diagnostic Category by Gender and Job Category*

\begin{tabular}{|c|c|c|c|c|c|}
\hline & & \multicolumn{4}{|c|}{ Women } \\
\hline & & \multicolumn{3}{|c|}{ Job Category } & \multirow[b]{2}{*}{ TOTAI } \\
\hline & & Service & Security and Fire & Crafts & \\
\hline Diagnostic Category & ICD-9-CM Code & \multirow[b]{2}{*}{0} & \multirow[b]{2}{*}{6} & \multirow[b]{2}{*}{0} & \multirow[b]{2}{*}{42} \\
\hline INFECTIOUS \& PARASITIC DISEASES (DIS) & 001-139 & & & & \\
\hline MALIGNANT NEOPLASMS & \begin{tabular}{|l|}
$140-208$ \\
$209.0-209.3$ \\
$230-234$
\end{tabular} & 0 & 0 & 0 & 41 \\
\hline BENIGN \& UNCERTAIN NEOPLASMS & $\begin{array}{l}\text { 209.4-209.6, } \\
210-229,235-239\end{array}$ & 0 & 0 & 0 & 184 \\
\hline ENDOCRINE/METABOLIC/IMMUNITY & 240-279 & 550 & 0 & 0 & 557 \\
\hline MENTAL DISORDERS & 290-319 & 71 & 0 & 0 & 298 \\
\hline NERVOUS SYSTEM (NS) \& SENSE ORGANS & 320-389 & 10 & 7 & 6 & 388 \\
\hline CIRCULATORY SYSTEM & $390-459$ & 543 & 0 & 0 & 575 \\
\hline RESPIRATORY SYSTEM & $460-519$ & 136 & 19 & 3 & 356 \\
\hline DIGESTIVE SYSTEM & $520-579$ & 71 & 0 & 0 & 291 \\
\hline GENITOURINARY SYSTEM & $580-629$ & 11 & 0 & 0 & 127 \\
\hline SKIN AND SUBCUTANEOUS TISSUE & 680-709 & 0 & 0 & 0 & 22 \\
\hline $\begin{array}{l}\text { MUSCULOSKELETAL \& CONNECTIVE } \\
\text { TISSUE }\end{array}$ & 710-739 & 1,144 & 0 & 1 & 1,986 \\
\hline CONGENITAL ANOMALIES & 740-759 & 0 & 0 & 0 & 53 \\
\hline $\begin{array}{l}\text { SYMPTOMS, SIGNS, \& ILL-DEFINED } \\
\text { CONDITIONS }\end{array}$ & 780-799 & 25 & 0 & 0 & 95 \\
\hline INJURY \& POISONING & 800-999 & 564 & 0 & 0 & 1,092 \\
\hline
\end{tabular}

\footnotetext{
*Absences with >1 ICD-9-CM code in the same diagnostic category were counted only once. Only those diagnostic categories and gender/job category combinations with at least one occurrence appear in this table.
} 
Brookhaven National Laboratory 2010

Absence Data

Appendix J. Total Number of Calendar Days Absent in Each Diagnostic Category by Gender and Job Category*

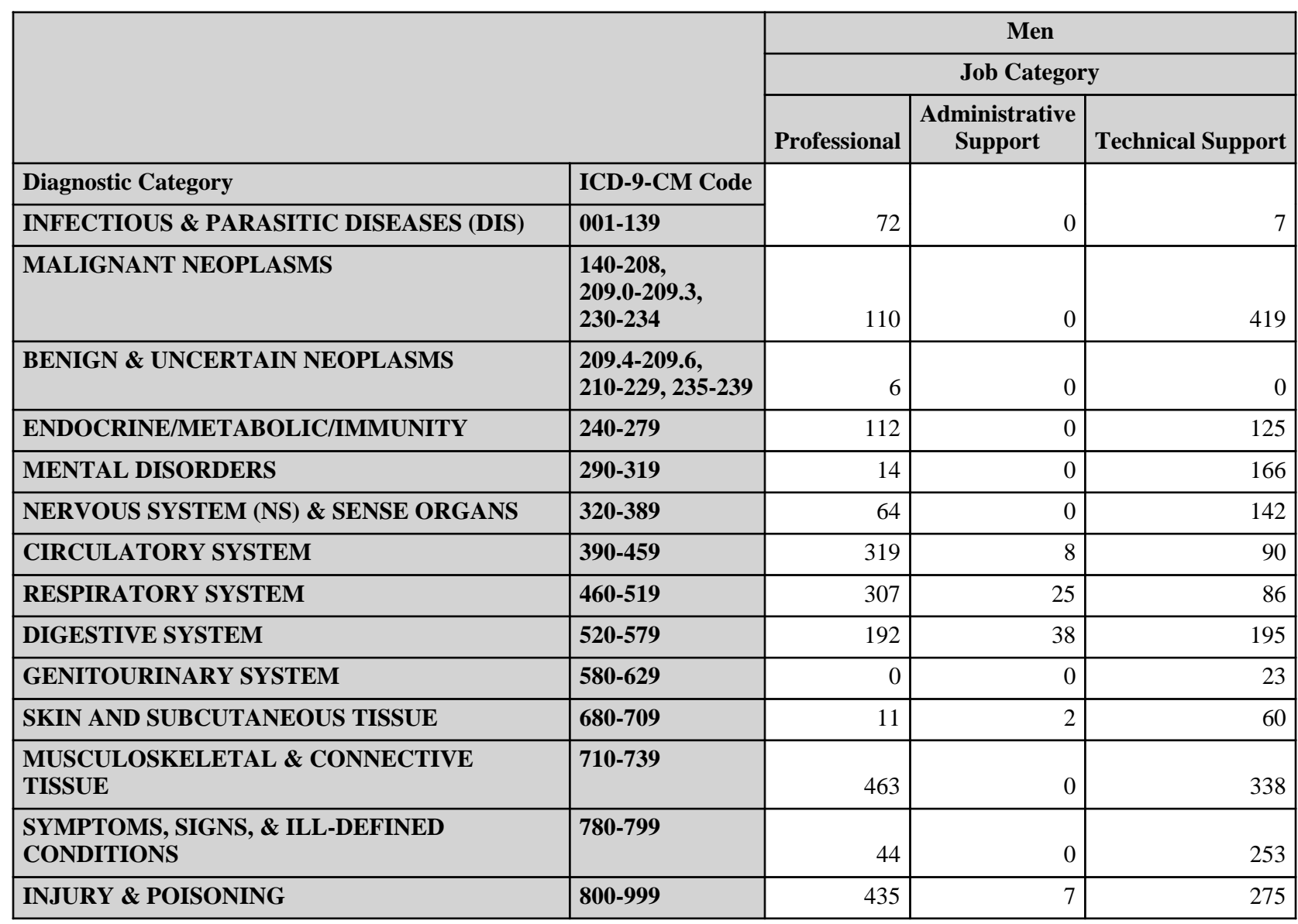

\footnotetext{
*Absences with >1 ICD-9-CM code in the same diagnostic category were counted only once. Only those diagnostic categories and gender/job category combinations with at least one occurrence appear in this table.
} 
Brookhaven National Laboratory 2010

Absence Data

Appendix J. Total Number of Calendar Days Absent in Each Diagnostic Category by Gender and Job Category*

\begin{tabular}{|c|c|c|c|c|c|}
\hline & & \multicolumn{4}{|c|}{ Men } \\
\hline & & \multicolumn{3}{|c|}{ Job Category } & \multirow[b]{2}{*}{ TOTAL } \\
\hline & & Service & Security and Fire & Crafts & \\
\hline Diagnostic Category & ICD-9-CM Code & \multirow[b]{2}{*}{12} & \multirow[b]{2}{*}{6} & \multirow[b]{2}{*}{26} & \multirow[b]{2}{*}{123} \\
\hline INFECTIOUS \& PARASITIC DISEASES (DIS) & 001-139 & & & & \\
\hline MALIGNANT NEOPLASMS & $\begin{array}{l}140-208 \\
209.0-209.3 \\
230-234\end{array}$ & 36 & 41 & 0 & 606 \\
\hline BENIGN \& UNCERTAIN NEOPLASMS & $\begin{array}{l}209.4-209.6 \\
210-229,235-239\end{array}$ & 0 & 0 & 0 & 6 \\
\hline ENDOCRINE/METABOLIC/IMMUNITY & $240-279$ & 54 & 0 & 7 & 298 \\
\hline MENTAL DISORDERS & $290-319$ & 8 & 15 & 0 & 203 \\
\hline NERVOUS SYSTEM (NS) \& SENSE ORGANS & 320-389 & 178 & 18 & 438 & 840 \\
\hline CIRCULATORY SYSTEM & $390-459$ & 164 & 173 & 10 & 764 \\
\hline RESPIRATORY SYSTEM & $460-519$ & 98 & 23 & 93 & 632 \\
\hline DIGESTIVE SYSTEM & $520-579$ & 275 & 14 & 138 & 852 \\
\hline GENITOURINARY SYSTEM & $580-629$ & 0 & 4 & 28 & 55 \\
\hline SKIN AND SUBCUTANEOUS TISSUE & $680-709$ & 43 & 0 & 6 & 122 \\
\hline $\begin{array}{l}\text { MUSCULOSKELETAL \& CONNECTIVE } \\
\text { TISSUE }\end{array}$ & 710-739 & 651 & 42 & 357 & 1,851 \\
\hline $\begin{array}{l}\text { SYMPTOMS, SIGNS, \& ILL-DEFINED } \\
\text { CONDITIONS }\end{array}$ & 780-799 & 21 & 8 & 18 & 344 \\
\hline INJURY \& POISONING & 800-999 & 518 & 96 & 485 & 1,816 \\
\hline
\end{tabular}

\footnotetext{
*Absences with >1 ICD-9-CM code in the same diagnostic category were counted only once. Only those diagnostic categories and gender/job category combinations with at least one occurrence appear in this table.
} 


\section{Brookhaven National Laboratory 2010}

Absence Data

Appendix K. Age-Adjusted Illness and Injury Rates by Diagnostic Category*

Part 1. Men

\begin{tabular}{|c|c|c|c|c|c|}
\hline & & $\begin{array}{l}\text { Number of } \\
\text { Diagnoses }\end{array}$ & $\begin{array}{c}\text { Age-Adjusted } \\
\text { Rate per } 1,000 * *\end{array}$ & \begin{tabular}{c|} 
Lower $95 \%$ \\
Confidence \\
Limit per 1,000
\end{tabular} & $\begin{array}{c}\text { Upper } 95 \% \\
\text { Confidence } \\
\text { Limit per 1,000 }\end{array}$ \\
\hline Diagnostic Category & ICD-9-CM Code & & & & \\
\hline INFECTIOUS \& PARASITIC DISEASES (DIS) & 001-139 & 16 & 7.8 & 4.3 & 14.1 \\
\hline -Other Bacterial Dis & $030-041$ & 6 & 2.8 & 1.2 & 6.6 \\
\hline -Viral Dis with Exanthem & 050-059 & 1 & 0.4 & 0.1 & 2.7 \\
\hline -Other Viral Dis \& Chlamydiae & 070-079 & 5 & 2.8 & 0.8 & 9.5 \\
\hline -Rickettsioses \& Other Arthropod & $080-088$ & 3 & 1.4 & 0.4 & 4.7 \\
\hline -Other Infections \& Parasitic Dis & 130-136 & 1 & 0.4 & 0.1 & 2.7 \\
\hline MALIGNANT NEOPLASMS & $\begin{array}{l}140-208, \\
209.0-209.3 \\
230-234\end{array}$ & 14 & 3.6 & 2.1 & 6.1 \\
\hline -Lip, Oral Cavity, Pharynx & $140-149$ & 1 & 0.2 & 0.0 & 1.6 \\
\hline -Digestive \& Peritoneal & 150-159 & 2 & 0.5 & 0.1 & 2.1 \\
\hline -Respiratory \& Intrathoracic & $160-165$ & 2 & 0.4 & 0.1 & 1.8 \\
\hline -Bone, Connective Tissue, Skin & $170-173,176$ & 3 & 0.9 & 0.3 & 2.7 \\
\hline -Genitourinary & 179-189 & 4 & 1.0 & 0.4 & 2.9 \\
\hline -Other \& Unspecified Sites & 195-199, 209.7 & 1 & 0.3 & 0.0 & 1.9 \\
\hline -Lymphatic \& Hematopoietic & $200-208$ & 1 & 0.3 & 0.0 & 1.9 \\
\hline BENIGN \& UNCERTAIN NEOPLASMS & \begin{tabular}{|l|}
$209.4-209.6$ \\
$210-229$, \\
$235-239$
\end{tabular} & 1 & 0.4 & 0.1 & 2.7 \\
\hline ENDOCRINE/METABOLIC/IMMUNITY & $240-279$ & 12 & 3.6 & 2.0 & 6.4 \\
\hline -Thyroid Gland Disorders & $240-246$ & 1 & 0.4 & 0.1 & 2.7 \\
\hline -Other Endocrine Gland Dis & $249-259$ & 7 & 2.0 & 0.9 & 4.2 \\
\hline -Other Metabolic \& Immunity Disorders & $270-279$ & 4 & 1.2 & 0.5 & 3.4 \\
\hline MENTAL DISORDERS & $290-319$ & 7 & 2.2 & 1.0 & 4.6 \\
\hline -Psychoses & 290-299 & 1 & 0.2 & 0.0 & 1.6 \\
\hline -Non-Psychotic Disorders & $300-302,306-316$ & 6 & 1.9 & 0.9 & 4.4 \\
\hline NERVOUS SYSTEM (NS) \& SENSE ORGANS & $320-389$ & 27 & 9.6 & 5.9 & 15.4 \\
\hline -Hereditary/Degenerative Central NS Dis & 330-337 & 1 & 0.2 & 0.0 & 1.6 \\
\hline -Other Disorders of Central NS & $340-349$ & 2 & 1.9 & 0.3 & 10.6 \\
\hline -Disorders of Peripheral NS & 350-359 & 7 & 2.2 & 1.0 & 4.9 \\
\hline -Disorders of Eye & $360-379$ & 13 & 3.8 & 2.2 & 6.8 \\
\hline -Diseases of Ear \& Mastoid & $380-389$ & 4 & 1.4 & 0.5 & 4.2 \\
\hline CIRCULATORY SYSTEM & $390-459$ & 29 & 7.5 & 5.2 & 10.8 \\
\hline -Hypertensive Dis & 401-405 & 6 & 1.6 & 0.7 & 3.6 \\
\hline -Ischemic Heart Dis & $410-414$ & 10 & 2.6 & 1.4 & 4.8 \\
\hline -Other Heart Dis & $420-429$ & 11 & 2.9 & 1.6 & 5.2 \\
\hline -Dis of Veins, Lymphatics, Other & 451-459 & 2 & 0.5 & 0.1 & 1.9 \\
\hline
\end{tabular}

(Continued)

*Only those diagnostic categories with at least one occurrence appear in this table.

**Standardized to age distribution of 2000 U.S. population. 


\section{Brookhaven National Laboratory 2010}

Absence Data

Appendix K. Age-Adjusted Illness and Injury Rates by Diagnostic Category*

Part 1. Men

\begin{tabular}{|c|c|c|c|c|c|}
\hline & & $\begin{array}{c}\text { Number of } \\
\text { Diagnoses }\end{array}$ & $\begin{array}{c}\text { Age-Adjusted } \\
\text { Rate per } 1,000^{* *}\end{array}$ & \begin{tabular}{c|} 
Lower $95 \%$ \\
Confidence \\
Limit per 1,000
\end{tabular} & $\begin{array}{c}\text { Upper } 95 \% \\
\text { Confidence } \\
\text { Limit per 1,000 }\end{array}$ \\
\hline Diagnostic Category & ICD-9-CM Code & & & & \\
\hline RESPIRATORY SYSTEM & $460-519$ & 55 & 23.5 & 16.7 & 33.3 \\
\hline -Acute Respiratory Infections & $460-466$ & 28 & 10.7 & 6.8 & 16.9 \\
\hline -Other Dis Upper Respiratory Tract & $470-478$ & 9 & 5.6 & 2.4 & 13.4 \\
\hline -Pneumonia \& Influenza & $480-488$ & 4 & 1.3 & 0.4 & 3.8 \\
\hline -Chronic Obstructive Dis & $490-496$ & 12 & 5.3 & 2.5 & 11.2 \\
\hline -Other Respiratory Dis & 510-519 & 2 & 0.6 & 0.2 & 2.6 \\
\hline DIGESTIVE SYSTEM & 520-579 & 52 & 21.5 & 15.2 & 30.5 \\
\hline -Oral Cavity, Saliva Glands, Jaw & $520-529$ & 3 & 1.0 & 0.3 & 3.2 \\
\hline -Esophagus, Stomach, Duodenum & 530-538 & 1 & 0.2 & 0.0 & 1.6 \\
\hline -Appendicitis & $540-543$ & 3 & 3.5 & 0.9 & 12.9 \\
\hline -Hernias & $\begin{array}{l}550-553 \\
\end{array}$ & 13 & 3.6 & 2.0 & 6.5 \\
\hline -Enteritis, Colitis & $555-558$ & 18 & 8.8 & 5.1 & 15.3 \\
\hline -Other Intestinal Dis & 560-569 & 9 & 3.0 & 1.5 & 6.1 \\
\hline -Other Digestive Dis & 570-579 & 5 & 1.3 & 0.5 & 3.3 \\
\hline GENITOURINARY SYSTEM & $580-629$ & 9 & 2.7 & 1.4 & 5.3 \\
\hline -Other Urinary Dis & 590-599 & 7 & 2.1 & 1.0 & 4.5 \\
\hline -Male Genital Organ Dis & $600-608$ & 2 & 0.6 & 0.1 & 2.5 \\
\hline SKIN AND SUBCUTANEOUS TISSUE & $680-709$ & 7 & 1.8 & 0.8 & 3.8 \\
\hline -Infections & $680-686$ & 2 & 0.5 & 0.1 & 1.9 \\
\hline -Other Inflammatory Conditions & 690-698 & 2 & 0.6 & 0.2 & 2.6 \\
\hline -Other & 700-709 & 3 & 0.7 & 0.2 & 2.1 \\
\hline $\begin{array}{l}\text { MUSCULOSKELETAL \& CONNECTIVE } \\
\text { TISSUE }\end{array}$ & 710-739 & 80 & 25.2 & 19.6 & 32.4 \\
\hline -Arthropathies & 710-719 & 33 & 10.6 & 7.4 & 15.2 \\
\hline -Dorsopathies & 720-724 & 36 & 11.7 & 7.8 & 17.6 \\
\hline -Rheumatism, Excluding Back & 725-729 & 10 & 2.6 & 1.4 & 4.8 \\
\hline -Other Dis \& Acquired Deformities & 730-739 & 1 & 0.4 & 0.1 & 2.7 \\
\hline $\begin{array}{l}\text { SYMPTOMS, SIGNS, \& ILL-DEFINED } \\
\text { CONDITIONS }\end{array}$ & 780-799 & 33 & 11.5 & 8.0 & 16.7 \\
\hline -Symptoms & 780-789 & 30 & 10.1 & 6.9 & 14.9 \\
\hline -Non-Specific Abnormal Findings & $790-796$ & 3 & 1.4 & 0.4 & 4.7 \\
\hline INJURY \& POISONING & $800-999$ & 67 & 31.9 & 23.0 & 44.2 \\
\hline -Fracture - Skull & $800-804$ & 1 & 0.4 & 0.1 & 2.7 \\
\hline -Fracture - Upper Limb & 810-819 & 10 & 7.7 & 3.5 & 16.7 \\
\hline -Fracture - Lower Limb & $820-829$ & 4 & 1.2 & 0.5 & 3.4 \\
\hline -Sprains \& Strains - Back & 846-847 & 5 & 1.9 & 0.8 & 4.8 \\
\hline
\end{tabular}

(Continued)

*Only those diagnostic categories with at least one occurrence appear in this table.

**Standardized to age distribution of 2000 U.S. population. 
Brookhaven National Laboratory 2010

Absence Data

Appendix K. Age-Adjusted Illness and Injury Rates by Diagnostic Category*

Part 1. Men

\begin{tabular}{|c|c|c|c|c|c|}
\hline & & $\begin{array}{l}\text { Number of } \\
\text { Diagnoses }\end{array}$ & $\begin{array}{c}\text { Age-Adjusted } \\
\text { Rate per } 1,000 * *\end{array}$ & \begin{tabular}{|c|} 
Lower $95 \%$ \\
Confidence \\
Limit per 1,000
\end{tabular} & $\begin{array}{c}\text { Upper } 95 \% \\
\text { Confidence } \\
\text { Limit per 1,000 }\end{array}$ \\
\hline Diagnostic Category & ICD-9-CM Code & \multirow[b]{2}{*}{27} & \multirow[b]{2}{*}{9.5} & \multirow[b]{2}{*}{5.8} & \multirow[b]{2}{*}{15.3} \\
\hline -Sprains \& Strains - Other & $840-845,848$ & & & & \\
\hline -Intracranial Injury & $850-854$ & 1 & 1.6 & 0.2 & 11.6 \\
\hline -Internal Injury - Thorax, Abdomen, Pelvis & $860-869$ & 2 & 3.3 & 0.8 & 13.1 \\
\hline -Open Wound - Head, Neck, Trunk & $870-879$ & 9 & 2.6 & 1.3 & 5.2 \\
\hline -Injury to Blood Vessels & $900-904$ & 1 & 0.2 & 0.0 & 1.6 \\
\hline -Contusion & $920-924$ & 2 & 0.6 & 0.2 & 2.6 \\
\hline -Crushing Injury & $925-929$ & 2 & 1.9 & 0.3 & 10.5 \\
\hline -Foreign Body Entering Orifice & 930-939 & 1 & 0.4 & 0.1 & 2.7 \\
\hline -Complications of Surgical/Medical Care & 996-999 & 2 & 0.5 & 0.1 & 2.1 \\
\hline Total & & 409 & 152.8 & 135.1 & 172.9 \\
\hline
\end{tabular}

* Only those diagnostic categories with at least one occurrence appear in this table.

**Standardized to age distribution of 2000 U.S. population. 


\section{Brookhaven National Laboratory 2010}

Absence Data

Appendix K. Age-Adjusted Illness and Injury Rates by Diagnostic Category*

Part 2. Women

\begin{tabular}{|c|c|c|c|c|c|}
\hline & & $\begin{array}{l}\text { Number of } \\
\text { Diagnoses }\end{array}$ & $\begin{array}{c}\text { Age-Adjusted } \\
\text { Rate per } 1,000 * *\end{array}$ & $\begin{array}{c}\text { Lower } 95 \% \\
\text { Confidence } \\
\text { Limit per 1,000 }\end{array}$ & $\begin{array}{c}\text { Upper } 95 \% \\
\text { Confidence } \\
\text { Limit per 1,000 }\end{array}$ \\
\hline Diagnostic Category & ICD-9-CM Code & & & & \\
\hline INFECTIOUS \& PARASITIC DISEASES (DIS) & 001-139 & 6 & 5.1 & 2.2 & 11.4 \\
\hline -Other Viral Dis \& Chlamydiae & 070-079 & 5 & 4.1 & 1.7 & 10.0 \\
\hline -Rickettsioses \& Other Arthropod & 080-088 & 1 & 1.0 & 0.1 & 6.8 \\
\hline MALIGNANT NEOPLASMS & $\begin{array}{l}140-208, \\
209.0-209.3 \\
230-234\end{array}$ & 2 & 1.2 & 0.3 & 4.9 \\
\hline -Breast & 174-175 & 1 & 0.6 & 0.1 & 4.3 \\
\hline -Genitourinary & $179-189$ & 1 & 0.6 & 0.1 & 4.3 \\
\hline BENIGN \& UNCERTAIN NEOPLASMS & \begin{tabular}{|l|}
$209.4-209.6$ \\
$210-229,235-239$
\end{tabular} & 5 & 6.0 & 2.4 & 15.3 \\
\hline ENDOCRINE/METABOLIC/IMMUNITY & $240-279$ & 3 & 3.3 & 1.0 & 11.3 \\
\hline -Other Endocrine Gland Dis & $249-259$ & 2 & 2.3 & 0.5 & 10.9 \\
\hline -Other Metabolic \& Immunity Disorders & $270-279$ & 1 & 1.0 & 0.1 & 6.8 \\
\hline MENTAL DISORDERS & 290-319 & 10 & 11.3 & 5.7 & 22.3 \\
\hline -Psychoses & 290-299 & 1 & 1.7 & 0.2 & 12.3 \\
\hline -Non-Psychotic Disorders & $300-302,306-316$ & 7 & 7.2 & 3.2 & 16.3 \\
\hline -Alcohol Dependence & 303 & 1 & 0.6 & 0.1 & 4.3 \\
\hline -Drug Dependence & 304-305 & 1 & 1.7 & 0.2 & 12.3 \\
\hline NERVOUS SYSTEM (NS) \& SENSE ORGANS & $320-389$ & 17 & 18.0 & 10.7 & 30.2 \\
\hline -Other Disorders of Central NS & $340-349$ & 2 & 2.3 & 0.5 & 10.9 \\
\hline -Disorders of Peripheral NS & $350-359$ & 7 & 6.2 & 2.8 & 13.9 \\
\hline -Disorders of Eye & $360-379$ & 1 & 1.7 & 0.2 & 12.3 \\
\hline -Diseases of Ear \& Mastoid & $380-389$ & 7 & 7.7 & 3.5 & 17.0 \\
\hline CIRCULATORY SYSTEM & $390-459$ & 7 & 7.8 & 3.5 & 17.2 \\
\hline -Hypertensive Dis & $401-405$ & 3 & 4.1 & 1.2 & 13.8 \\
\hline -Other Heart Dis & $420-429$ & 1 & 1.1 & 0.1 & 7.5 \\
\hline -Dis of Arteries \& Capillaries & $440-449$ & 1 & 1.1 & 0.1 & 7.5 \\
\hline -Dis of Veins, Lymphatics, Other & 451-459 & 2 & 1.6 & 0.4 & 6.5 \\
\hline RESPIRATORY SYSTEM & $460-519$ & 56 & 61.1 & 44.2 & 84.5 \\
\hline -Acute Respiratory Infections & $460-466$ & 11 & 14.7 & 6.7 & 32.0 \\
\hline -Other Dis Upper Respiratory Tract & $470-478$ & 17 & 19.4 & 10.4 & 36.2 \\
\hline -Pneumonia \& Influenza & $480-488$ & 5 & 5.0 & 1.9 & 12.9 \\
\hline -Chronic Obstructive Dis & $490-496$ & 22 & 21.5 & 13.8 & 33.5 \\
\hline -Other Respiratory Dis & $510-519$ & 1 & 0.6 & 0.1 & 4.3 \\
\hline DIGESTIVE SYSTEM & $520-579$ & 13 & 16.9 & 9.5 & 29.9 \\
\hline -Oral Cavity, Saliva Glands, Jaw & $520-529$ & 1 & 1.7 & 0.2 & 12.3 \\
\hline
\end{tabular}

(Continued)

*Only those diagnostic categories with at least one occurrence appear in this table.

**Standardized to age distribution of 2000 U.S. population. 


\section{Brookhaven National Laboratory 2010}

Absence Data

Appendix K. Age-Adjusted Illness and Injury Rates by Diagnostic Category*

Part 2. Women

\begin{tabular}{|c|c|c|c|c|c|}
\hline & & $\begin{array}{c}\text { Number of } \\
\text { Diagnoses }\end{array}$ & $\begin{array}{c}\text { Age-Adjusted } \\
\text { Rate per } 1,000^{* *}\end{array}$ & \begin{tabular}{c|} 
Lower $95 \%$ \\
Confidence \\
Limit per 1,000
\end{tabular} & $\begin{array}{c}\text { Upper } 95 \% \\
\text { Confidence } \\
\text { Limit per 1,000 }\end{array}$ \\
\hline Diagnostic Category & ICD-9-CM Code & & & & \\
\hline -Appendicitis & $540-543$ & 2 & 3.5 & 0.9 & 13.9 \\
\hline -Hernias & $\begin{array}{l}550-553 \\
\end{array}$ & 1 & 1.0 & 0.1 & 6.8 \\
\hline -Enteritis, Colitis & $555-558$ & 4 & 3.9 & 1.5 & 10.5 \\
\hline -Other Intestinal Dis & $560-569$ & 3 & 4.4 & 1.4 & 14.2 \\
\hline -Other Digestive Dis & $\begin{array}{l}570-579 \\
\end{array}$ & 2 & 2.3 & 0.5 & 10.9 \\
\hline GENITOURINARY SYSTEM & $580-629$ & 13 & 14.6 & 8.2 & 26.2 \\
\hline -Other Urinary Dis & 590-599 & 3 & 4.1 & 1.2 & 13.8 \\
\hline -Breast Disorders & $610-612$ & 4 & 4.0 & 1.4 & 11.8 \\
\hline -Other Female Disorders & $617-629$ & 6 & 6.5 & 2.9 & 15.0 \\
\hline SKIN AND SUBCUTANEOUS TISSUE & 680-709 & 2 & 1.7 & 0.4 & 7.0 \\
\hline -Infections & 680-686 & 1 & 0.6 & 0.1 & 4.3 \\
\hline -Other Inflammatory Conditions & 690-698 & 1 & 1.1 & 0.1 & 7.5 \\
\hline $\begin{array}{l}\text { MUSCULOSKELETAL \& CONNECTIVE } \\
\text { TISSUE }\end{array}$ & 710-739 & 52 & 52.7 & 39.2 & 70.8 \\
\hline -Arthropathies & 710-719 & 21 & 21.1 & 13.3 & 33.4 \\
\hline -Dorsopathies & 720-724 & 20 & 22.2 & 13.9 & 35.6 \\
\hline -Rheumatism, Excluding Back & 725-729 & 8 & 7.6 & 3.5 & 16.5 \\
\hline -Other Dis \& Acquired Deformities & 730-739 & 3 & 1.8 & 0.6 & 5.7 \\
\hline CONGENITAL ANOMALIES & 740-759 & 1 & 0.6 & 0.1 & 4.3 \\
\hline $\begin{array}{l}\text { SYMPTOMS, SIGNS, \& ILL-DEFINED } \\
\text { CONDITIONS }\end{array}$ & 780-799 & 30 & 32.7 & 22.3 & 47.8 \\
\hline -Symptoms & 780-789 & 29 & 32.1 & 21.8 & 47.2 \\
\hline -Non-Specific Abnormal Findings & 790-796 & 1 & 0.6 & 0.1 & 4.3 \\
\hline INJURY \& POISONING & $800-999$ & 28 & 26.1 & 17.4 & 39.2 \\
\hline -Fracture - Skull & 800-804 & 1 & 1.7 & 0.2 & 12.3 \\
\hline -Fracture - Upper Limb & $810-819$ & 5 & 3.4 & 1.4 & 8.3 \\
\hline -Fracture - Lower Limb & $820-829$ & 4 & 3.7 & 1.4 & 10.0 \\
\hline -Sprains \& Strains - Back & 846-847 & 1 & 1.7 & 0.2 & 12.3 \\
\hline -Sprains \& Strains - Other & $840-845,848$ & 5 & 3.9 & 1.6 & 9.8 \\
\hline -Open Wound - Head, Neck, Trunk & $870-879$ & 2 & 1.2 & 0.3 & 4.9 \\
\hline -Late Effects of Accident & $905-909$ & 1 & 0.6 & 0.1 & 4.3 \\
\hline -Contusion & $920-924$ & 4 & 2.4 & 0.9 & 6.5 \\
\hline -Unspecified Effects - External Causes & 990-995 & 3 & 4.5 & 1.4 & 14.4 \\
\hline -Complications of Surgical/Medical Care & 996-999 & 2 & 2.8 & 0.7 & 11.6 \\
\hline Total & & 245 & 259.0 & 225.0 & 298.1 \\
\hline
\end{tabular}

* Only those diagnostic categories with at least one occurrence appear in this table.

**Standardized to age distribution of 2000 U.S. population. 


\section{Brookhaven National Laboratory 2010}

Absence Data

Appendix K. Age-Adjusted Illness and Injury Rates by Diagnostic Category*

Part 3. Men and Women

\begin{tabular}{|c|c|c|c|c|c|}
\hline & & $\begin{array}{l}\text { Number of } \\
\text { Diagnoses }\end{array}$ & $\begin{array}{c}\text { Age-Adjusted } \\
\text { Rate per } 1,000 * *\end{array}$ & $\begin{array}{c}\text { Lower } 95 \% \\
\text { Confidence } \\
\text { Limit per 1,000 }\end{array}$ & $\begin{array}{c}\text { Upper } 95 \% \\
\text { Confidence } \\
\text { Limit per 1,000 }\end{array}$ \\
\hline Diagnostic Category & ICD-9-CM Code & & & & \\
\hline INFECTIOUS \& PARASITIC DISEASES (DIS) & 001-139 & 22 & 7.1 & 4.3 & 11.6 \\
\hline -Other Bacterial Dis & 030-041 & 6 & 2.1 & 0.9 & 4.9 \\
\hline -Viral Dis with Exanthem & 050-059 & 1 & 0.3 & 0.0 & 1.9 \\
\hline -Other Viral Dis \& Chlamydiae & 070-079 & 10 & 3.1 & 1.4 & 7.1 \\
\hline -Rickettsioses \& Other Arthropod & 080-088 & 4 & 1.3 & 0.5 & 3.7 \\
\hline -Other Infections \& Parasitic Dis & $130-136$ & 1 & 0.3 & 0.0 & 1.9 \\
\hline MALIGNANT NEOPLASMS & $\begin{array}{l}140-208, \\
209.0-209.3 \\
230-234\end{array}$ & 16 & 3.1 & 1.9 & 5.1 \\
\hline -Lip, Oral Cavity, Pharynx & $140-149$ & 1 & 0.2 & 0.0 & 1.2 \\
\hline -Digestive \& Peritoneal & 150-159 & 2 & 0.4 & 0.1 & 1.7 \\
\hline -Respiratory \& Intrathoracic & $160-165$ & 2 & 0.3 & 0.1 & 1.3 \\
\hline -Bone, Connective Tissue, Skin & 170-173, 176 & 3 & 0.7 & 0.2 & 2.1 \\
\hline -Breast & 174-175 & 1 & 0.2 & 0.0 & 1.2 \\
\hline -Genitourinary & 179-189 & 5 & 0.9 & 0.4 & 2.3 \\
\hline -Other \& Unspecified Sites & 195-199, 209.7 & 1 & 0.2 & 0.0 & 1.5 \\
\hline -Lymphatic \& Hematopoietic & $200-208$ & 1 & 0.2 & 0.0 & 1.5 \\
\hline BENIGN \& UNCERTAIN NEOPLASMS & $\begin{array}{l}209.4-209.6 \\
210-229,235-239\end{array}$ & 6 & 1.9 & 0.8 & 4.3 \\
\hline ENDOCRINE/METABOLIC/IMMUNITY & $240-279$ & 15 & 3.6 & 2.1 & 6.1 \\
\hline -Thyroid Gland Disorders & $240-246$ & 1 & 0.3 & 0.0 & 1.9 \\
\hline -Other Endocrine Gland Dis & 249-259 & 9 & 2.1 & 1.1 & 4.2 \\
\hline -Other Metabolic \& Immunity Disorders & 270-279 & 5 & 1.2 & 0.5 & 2.9 \\
\hline MENTAL DISORDERS & 290-319 & 17 & 4.5 & 2.7 & 7.5 \\
\hline -Psychoses & 290-299 & 2 & 0.6 & 0.1 & 2.8 \\
\hline -Non-Psychotic Disorders & $300-302,306-316$ & 13 & 3.3 & 1.9 & 5.9 \\
\hline -Alcohol Dependence & 303 & 1 & 0.2 & 0.0 & 1.2 \\
\hline -Drug Dependence & $304-305$ & 1 & 0.4 & 0.1 & 3.1 \\
\hline NERVOUS SYSTEM (NS) \& SENSE ORGANS & 320-389 & 44 & 11.8 & 8.4 & 16.7 \\
\hline -Hereditary/Degenerative Central NS Dis & 330-337 & 1 & 0.2 & 0.0 & 1.2 \\
\hline -Other Disorders of Central NS & $340-349$ & 4 & 1.9 & 0.5 & 6.7 \\
\hline -Disorders of Peripheral NS & $350-359$ & 14 & 3.3 & 1.9 & 5.8 \\
\hline -Disorders of Eye & $360-379$ & 14 & 3.4 & 2.0 & 6.0 \\
\hline -Diseases of Ear \& Mastoid & 380-389 & 11 & 3.0 & 1.6 & 5.7 \\
\hline CIRCULATORY SYSTEM & $390-459$ & 36 & 7.9 & 5.6 & 11.1 \\
\hline -Hypertensive Dis & $401-405$ & 9 & 2.3 & 1.1 & 4.6 \\
\hline
\end{tabular}

(Continued)

*Only those diagnostic categories with at least one occurrence appear in this table.

**Standardized to age distribution of 2000 U.S. population. 


\section{Brookhaven National Laboratory 2010}

\section{Absence Data}

Appendix K. Age-Adjusted IIIness and Injury Rates by Diagnostic Category*

Part 3. Men and Women

\begin{tabular}{|c|c|c|c|c|c|}
\hline & & $\begin{array}{c}\text { Number of } \\
\text { Diagnoses }\end{array}$ & $\begin{array}{c}\text { Age-Adjusted } \\
\text { Rate per } 1,000^{* *}\end{array}$ & \begin{tabular}{c|} 
Lower $95 \%$ \\
Confidence \\
Limit per 1,000
\end{tabular} & $\begin{array}{c}\text { Upper } 95 \% \\
\text { Confidence } \\
\text { Limit per 1,000 }\end{array}$ \\
\hline Diagnostic Category & ICD-9-CM Code & & & & \\
\hline -Ischemic Heart Dis & $410-414$ & 10 & 2.1 & 1.1 & 3.9 \\
\hline -Other Heart Dis & $420-429$ & 12 & 2.5 & 1.4 & 4.4 \\
\hline -Dis of Arteries \& Capillaries & $440-449$ & 1 & 0.2 & 0.0 & 1.5 \\
\hline -Dis of Veins, Lymphatics, Other & $451-459$ & 4 & 0.8 & 0.3 & 2.2 \\
\hline RESPIRATORY SYSTEM & $460-519$ & 111 & 33.8 & 26.6 & 42.9 \\
\hline -Acute Respiratory Infections & $460-466$ & 39 & 12.1 & 8.1 & 18.2 \\
\hline -Other Dis Upper Respiratory Tract & $470-478$ & 26 & 9.5 & 5.7 & 16.0 \\
\hline -Pneumonia \& Influenza & $480-488$ & 9 & 2.2 & 1.1 & 4.5 \\
\hline -Chronic Obstructive Dis & $490-496$ & 34 & 9.2 & 6.2 & 13.7 \\
\hline -Other Respiratory Dis & $510-519$ & 3 & 0.7 & 0.2 & 2.1 \\
\hline DIGESTIVE SYSTEM & 520-579 & 65 & 20.2 & 15.1 & 27.0 \\
\hline -Oral Cavity, Saliva Glands, Jaw & $520-529$ & 4 & 1.2 & 0.4 & 3.3 \\
\hline -Esophagus, Stomach, Duodenum & 530-538 & 1 & 0.2 & 0.0 & 1.2 \\
\hline -Appendicitis & $540-543$ & 5 & 3.3 & 1.2 & 9.1 \\
\hline -Hernias & $550-553$ & 14 & 3.0 & 1.7 & 5.2 \\
\hline -Enteritis, Colitis & 555-558 & 22 & 7.5 & 4.6 & 12.1 \\
\hline -Other Intestinal Dis & $560-569$ & 12 & 3.4 & 1.9 & 6.3 \\
\hline -Other Digestive Dis & $570-579$ & 7 & 1.6 & 0.7 & 3.6 \\
\hline GENITOURINARY SYSTEM & $580-629$ & 22 & 5.8 & 3.7 & 9.1 \\
\hline -Other Urinary Dis & 590-599 & 10 & 2.6 & 1.3 & 5.1 \\
\hline -Male Genital Organ Dis & $600-608$ & 2 & 0.4 & 0.1 & 1.8 \\
\hline -Breast Disorders & $610-612$ & 4 & 1.0 & 0.3 & 2.9 \\
\hline -Other Female Disorders & 617-629 & 6 & 1.8 & 0.8 & 4.1 \\
\hline SKIN AND SUBCUTANEOUS TISSUE & $680-709$ & 9 & 1.7 & 0.9 & 3.4 \\
\hline -Infections & $680-686$ & 3 & 0.5 & 0.2 & 1.7 \\
\hline -Other Inflammatory Conditions & 690-698 & 3 & 0.7 & 0.2 & 2.2 \\
\hline -Other & 700-709 & 3 & 0.5 & 0.2 & 1.5 \\
\hline $\begin{array}{l}\text { MUSCULOSKELETAL \& CONNECTIVE } \\
\text { TISSUE }\end{array}$ & 710-739 & 132 & 32.0 & 26.5 & 38.7 \\
\hline -Arthropathies & 710-719 & 54 & 13.0 & 9.8 & 17.3 \\
\hline -Dorsopathies & 720-724 & 56 & 14.4 & 10.7 & 19.5 \\
\hline -Rheumatism, Excluding Back & 725-729 & 18 & 3.8 & 2.3 & 6.3 \\
\hline -Other Dis \& Acquired Deformities & 730-739 & 4 & 0.8 & 0.3 & 2.1 \\
\hline CONGENITAL ANOMALIES & 740-759 & 1 & 0.2 & 0.0 & 1.2 \\
\hline $\begin{array}{l}\text { SYMPTOMS, SIGNS, \& ILL-DEFINED } \\
\text { CONDITIONS }\end{array}$ & 780-799 & 63 & 16.7 & 12.8 & 21.7 \\
\hline
\end{tabular}

(Continued)

*Only those diagnostic categories with at least one occurrence appear in this table.

**Standardized to age distribution of 2000 U.S. population. 
Brookhaven National Laboratory 2010

Absence Data

Appendix K. Age-Adjusted Illness and Injury Rates by Diagnostic Category*

Part 3. Men and Women

\begin{tabular}{|c|c|c|c|c|c|}
\hline & & $\begin{array}{l}\text { Number of } \\
\text { Diagnoses }\end{array}$ & $\begin{array}{c}\text { Age-Adjusted } \\
\text { Rate per 1,000** }\end{array}$ & $\begin{array}{c}\text { Lower } 95 \% \\
\text { Confidence } \\
\text { Limit per 1,000 }\end{array}$ & $\begin{array}{c}\text { Upper } 95 \% \\
\text { Confidence } \\
\text { Limit per } 1,000\end{array}$ \\
\hline Diagnostic Category & ICD-9-CM Code & \multirow[b]{2}{*}{59} & \multirow[b]{2}{*}{15.5} & \multirow[b]{2}{*}{11.8} & \multirow[b]{2}{*}{20.3} \\
\hline -Symptoms & $780-789$ & & & & \\
\hline -Non-Specific Abnormal Findings & $790-796$ & 4 & 1.2 & 0.4 & 3.5 \\
\hline INJURY \& POISONING & $800-999$ & 95 & 29.4 & 22.6 & 38.2 \\
\hline -Fracture - Skull & $800-804$ & 2 & 0.7 & 0.2 & 2.9 \\
\hline -Fracture - Upper Limb & $810-819$ & 15 & 6.4 & 3.3 & 12.3 \\
\hline -Fracture - Lower Limb & $820-829$ & 8 & 1.8 & 0.9 & 3.6 \\
\hline -Sprains \& Strains - Back & 846-847 & 6 & 1.9 & 0.8 & 4.3 \\
\hline -Sprains \& Strains - Other & $840-845,848$ & 32 & 7.8 & 5.1 & 12.0 \\
\hline -Intracranial Injury & $850-854$ & 1 & 1.1 & 0.2 & 8.0 \\
\hline -Internal Injury - Thorax, Abdomen, Pelvis & $860-869$ & 2 & 2.2 & 0.6 & 9.0 \\
\hline -Open Wound - Head, Neck, Trunk & 870-879 & 11 & 2.3 & 1.2 & 4.2 \\
\hline -Injury to Blood Vessels & $900-904$ & 1 & 0.2 & 0.0 & 1.2 \\
\hline -Late Effects of Accident & 905-909 & 1 & 0.2 & 0.0 & 1.2 \\
\hline -Contusion & $920-924$ & 6 & 1.1 & 0.5 & 2.6 \\
\hline -Crushing Injury & $925-929$ & 2 & 1.3 & 0.3 & 7.1 \\
\hline -Foreign Body Entering Orifice & $930-939$ & 1 & 0.3 & 0.0 & 1.9 \\
\hline -Unspecified Effects - External Causes & $990-995$ & 3 & 1.1 & 0.3 & 3.5 \\
\hline -Complications of Surgical/Medical Care & 996-999 & 4 & 1.1 & 0.4 & 3.1 \\
\hline Total & & 654 & 179.6 & 163.8 & 197.0 \\
\hline
\end{tabular}

* Only those diagnostic categories with at least one occurrence appear in this table.

**Standardized to age distribution of 2000 U.S. population. 
Brookhaven National Laboratory 2010

OSHA Data

Appendix L. Number of Workers with at Least One OSHA Event by Gender, Age, and Job Category*

\begin{tabular}{|c|c|c|c|c|c|c|c|c|c|}
\hline \multirow{3}{*}{ Job Category } & \multicolumn{3}{|c|}{ Women } & \multicolumn{5}{|c|}{ Men } & \multirow[b]{3}{*}{ TOTAL } \\
\hline & \multicolumn{2}{|c|}{ Age Group } & \multirow[b]{2}{*}{ TOTAL } & \multicolumn{4}{|c|}{ Age Group } & \multirow[b]{2}{*}{ TOTAL } & \\
\hline & $30-39$ & $50+$ & & $16-29$ & $30-39$ & $40-49$ & $50+$ & & \\
\hline Professional & 1 & 2 & 3 & 0 & 0 & 0 & 2 & 2 & 5 \\
\hline Administrative Support & 1 & 1 & 2 & 0 & 0 & 0 & 0 & 0 & 2 \\
\hline Technical Support & 0 & 0 & 0 & 1 & 0 & 3 & 0 & 4 & 4 \\
\hline Service & 0 & 0 & 0 & 0 & 0 & 1 & 4 & 5 & 5 \\
\hline Security and Fire & 0 & 0 & 0 & 0 & 0 & 3 & 1 & 4 & 4 \\
\hline Crafts & 0 & 0 & 0 & 2 & 1 & 5 & 1 & 9 & 9 \\
\hline TOTAL & 2 & 3 & 5 & 3 & 1 & 12 & 8 & 24 & 29 \\
\hline
\end{tabular}

*Only those job categories and gender/age combinations with at least one OSHA event appear in this table.

Appendix M. Total Number of Workdays Lost or with Restricted Activity from OSHA Events by Gender and Age

\begin{tabular}{|c|c|c|c|c|c|c|c|c|c|}
\hline \multirow[b]{2}{*}{ Age Group } & \multicolumn{3}{|c|}{ Women } & \multicolumn{3}{|c|}{ Men } & \multicolumn{3}{|c|}{ TOTAL } \\
\hline & $\begin{array}{c}\text { Number of } \\
\text { Events }\end{array}$ & \begin{tabular}{|c} 
Days \\
Restricted
\end{tabular} & Days Lost & $\begin{array}{c}\text { Number of } \\
\text { Events }\end{array}$ & \begin{tabular}{|c|} 
Days \\
Restricted
\end{tabular} & Days Lost & \begin{tabular}{|c|} 
Number of \\
Events
\end{tabular} & $\begin{array}{c}\text { Days } \\
\text { Restricted }\end{array}$ & Days Lost \\
\hline $16-29$ & 0 & 0 & 0 & 3 & 32 & 50 & 3 & 32 & 50 \\
\hline 30 - 39 & 2 & 0 & 0 & 1 & 6 & 6 & 3 & 6 & 6 \\
\hline $40-49$ & 0 & 0 & 0 & 12 & 110 & 63 & 12 & 110 & 63 \\
\hline $50+$ & 3 & 0 & 3 & 8 & 88 & 147 & 11 & 88 & 150 \\
\hline TOTAL & 5 & 0 & 3 & 24 & 236 & 266 & 29 & 236 & 269 \\
\hline
\end{tabular}

Appendix N. Total Number of Workdays Lost or with Restricted Activity from OSHA Events by Gender and Job Category*

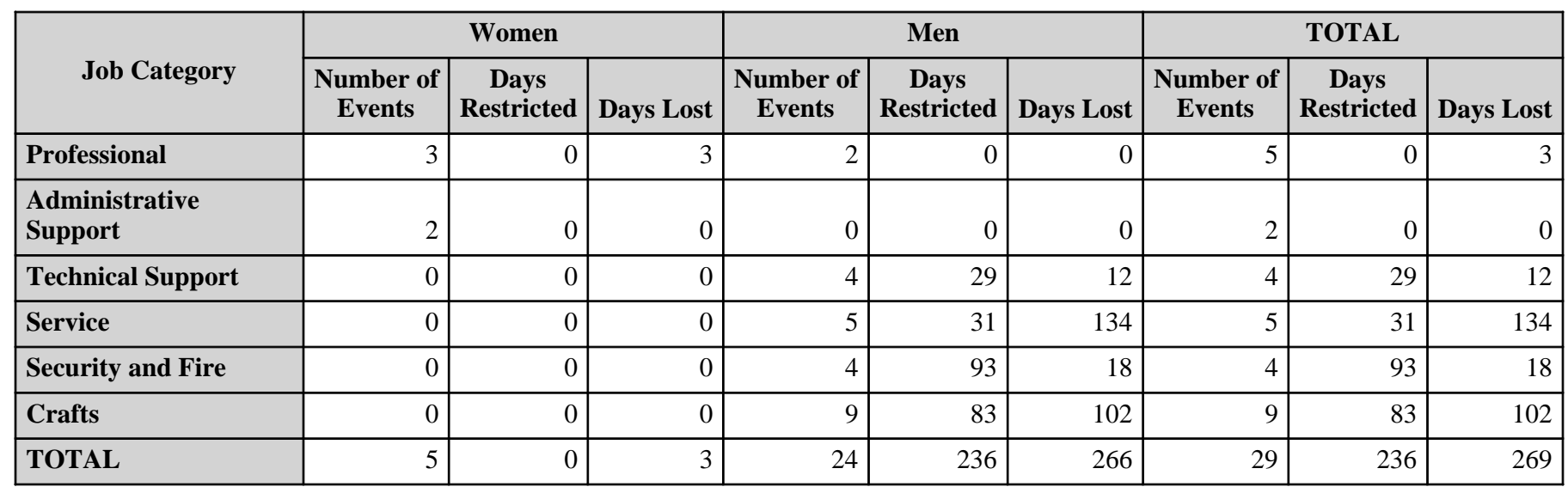

*Only those job categories with at least one OSHA event appear in this table. 
Brookhaven National Laboratory 2010

OSHA Data

Appendix O. Number of Diagnoses in Each Diagnostic Category by Gender and Age*

\begin{tabular}{|c|c|c|c|c|}
\hline & & \multicolumn{3}{|c|}{ Women } \\
\hline & & \multicolumn{2}{|c|}{ Age Group } & \multirow[b]{2}{*}{ TOTAL } \\
\hline & & $30-39$ & $50+$ & \\
\hline Diagnostic Category & ICD-9-CM code & & & \\
\hline NERVOUS SYSTEM (NS) \& SENSE ORGANS & 320-389 & 0 & 0 & 0 \\
\hline -Disorders of Eye & $360-379$ & 0 & 0 & 0 \\
\hline $\begin{array}{l}\text { MUSCULOSKELETAL \& CONNECTIVE } \\
\text { TISSUE }\end{array}$ & 710-739 & 0 & 0 & 0 \\
\hline -Dorsopathies & $720-724$ & 0 & 0 & 0 \\
\hline INJURY \& POISONING & $800-999$ & 2 & 3 & 5 \\
\hline -Fracture - Upper Limb & 810-819 & 0 & 0 & 0 \\
\hline -Sprains \& Strains - Back & 846-847 & 0 & 0 & 0 \\
\hline -Sprains \& Strains - Other & $840-845,848$ & 0 & 0 & 0 \\
\hline -Open Wound - Head, Neck, Trunk & $870-879$ & 0 & 0 & 0 \\
\hline -Open Wound - Upper Limb & $880-887$ & 0 & 0 & 0 \\
\hline -Superficial Injury & $910-919$ & 0 & 0 & 0 \\
\hline -Crushing Injury & 925-929 & 0 & 0 & 0 \\
\hline -Burns & $940-949$ & 1 & 0 & 1 \\
\hline -Complications \& Unspecified Injuries & 958-959 & 1 & 2 & 3 \\
\hline -Toxic Effects - Non-medicinal & 980-989 & 0 & 1 & 1 \\
\hline
\end{tabular}

\begin{tabular}{|c|c|c|c|c|c|c|c|}
\hline & & \multicolumn{5}{|c|}{ Men } & \multirow[b]{3}{*}{ TOTAL } \\
\hline & & \multicolumn{4}{|c|}{ Age Group } & \multirow[b]{2}{*}{ TOTAL } & \\
\hline & & $16-29$ & 30 - 39 & $40-49$ & $50+$ & & \\
\hline Diagnostic Category & ICD-9-CM code & & & & & & \\
\hline NERVOUS SYSTEM (NS) \& SENSE ORGANS & 320-389 & 0 & 0 & 1 & 0 & 1 & 1 \\
\hline -Disorders of Eye & $360-379$ & 0 & 0 & 1 & 0 & 1 & 1 \\
\hline $\begin{array}{l}\text { MUSCULOSKELETAL \& CONNECTIVE } \\
\text { TISSUE }\end{array}$ & 710-739 & 0 & 0 & 0 & 1 & 1 & 1 \\
\hline -Dorsopathies & $720-724$ & 0 & 0 & 0 & 1 & 1 & 1 \\
\hline INJURY \& POISONING & $800-999$ & 3 & 1 & 11 & 7 & 22 & 27 \\
\hline -Fracture - Upper Limb & $810-819$ & 1 & 0 & 0 & 0 & 1 & 1 \\
\hline -Sprains \& Strains - Back & 846-847 & 0 & 0 & 1 & 0 & 1 & 1 \\
\hline -Sprains \& Strains - Other & $840-845,848$ & 1 & 0 & 1 & 0 & 2 & 2 \\
\hline -Open Wound - Head, Neck, Trunk & $870-879$ & 0 & 0 & 0 & 1 & 1 & 1 \\
\hline -Open Wound - Upper Limb & $880-887$ & 0 & 0 & 2 & 0 & 2 & 2 \\
\hline -Superficial Injury & 910-919 & 0 & 0 & 1 & 0 & 1 & 1 \\
\hline -Crushing Injury & 925-929 & 1 & 0 & 0 & 0 & 1 & 1 \\
\hline -Burns & $940-949$ & 0 & 0 & 0 & 1 & 1 & 2 \\
\hline -Complications \& Unspecified Injuries & 958-959 & 0 & 1 & 6 & 5 & 12 & 15 \\
\hline -Toxic Effects - Non-medicinal & 980-989 & 0 & 0 & 0 & 0 & 0 & 1 \\
\hline
\end{tabular}

*Only those diagnostic categories and gender/age combinations with at least one OSHA event appear in this table. 
Brookhaven National Laboratory 2010

OSHA Data

Appendix O. Number of Diagnoses in Each Diagnostic Category by Gender and Age*

\begin{tabular}{|c|c|c|c|c|c|c|c|c|c|}
\hline & \multicolumn{3}{|c|}{ Women } & \multicolumn{5}{|c|}{ Men } & \multirow[b]{3}{*}{ TOTAL } \\
\hline & \multicolumn{2}{|c|}{ Age Group } & \multirow[b]{2}{*}{ TOTAL } & \multicolumn{4}{|c|}{ Age Group } & \multirow[b]{2}{*}{ TOTAL } & \\
\hline & 30 - 39 & $50+$ & & $16-29$ & 30 - 39 & $40-49$ & $50+$ & & \\
\hline Diagnostic Category & \multirow[b]{2}{*}{2} & \multirow[b]{2}{*}{3} & \multirow[b]{2}{*}{5} & \multirow[b]{2}{*}{3} & \multirow[b]{2}{*}{1} & \multirow[b]{2}{*}{12} & \multirow[b]{2}{*}{8} & \multirow[b]{2}{*}{24} & \multirow[b]{2}{*}{29} \\
\hline Total & & & & & & & & & \\
\hline
\end{tabular}

*Only those diagnostic categories and gender/age combinations with at least one OSHA event appear in this table. 
Brookhaven National Laboratory 2010

OSHA Data

Appendix P. Number of Workdays Lost or with Restricted Activity in Each Diagnostic Category by Gender and Age*

\begin{tabular}{|c|c|c|c|c|c|}
\hline & & \multicolumn{4}{|c|}{ Women } \\
\hline & & \multicolumn{4}{|c|}{ Age Group } \\
\hline & & \multicolumn{2}{|c|}{$30-39$} & \multicolumn{2}{|c|}{$50+$} \\
\hline & & $\begin{array}{c}\text { Days } \\
\text { Restricted }\end{array}$ & Days Lost & $\begin{array}{c}\text { Days } \\
\text { Restricted }\end{array}$ & Days Lost \\
\hline Diagnostic Category & ICD-9-CM Codes & \multirow[b]{2}{*}{0} & \multirow[b]{2}{*}{0} & \multirow[b]{2}{*}{0} & \multirow[b]{2}{*}{0} \\
\hline -Burns & $940-949$ & & & & \\
\hline $\begin{array}{l}\text {-Complications \& Unspecified } \\
\text { Injuries }\end{array}$ & 958-959 & 0 & 0 & 0 & 3 \\
\hline -Toxic Effects - Non-medicinal & $980-989$ & 0 & 0 & 0 & 0 \\
\hline
\end{tabular}

\begin{tabular}{|c|c|c|c|c|c|c|c|c|c|}
\hline & & \multicolumn{8}{|c|}{ Men } \\
\hline & & \multicolumn{8}{|c|}{ Age Group } \\
\hline & & \multicolumn{2}{|c|}{$16-29$} & \multicolumn{2}{|c|}{30 - 39} & \multicolumn{2}{|c|}{40 - 49} & \multicolumn{2}{|c|}{$50+$} \\
\hline & & $\begin{array}{c}\text { Days } \\
\text { Restricted }\end{array}$ & $\begin{array}{l}\text { Days } \\
\text { Lost }\end{array}$ & $\begin{array}{c}\text { Days } \\
\text { Restricted }\end{array}$ & $\begin{array}{l}\text { Days } \\
\text { Lost }\end{array}$ & $\begin{array}{c}\text { Days } \\
\text { Restricted }\end{array}$ & $\begin{array}{l}\text { Days } \\
\text { Lost }\end{array}$ & $\begin{array}{c}\text { Days } \\
\text { Restricted }\end{array}$ & $\begin{array}{l}\text { Days } \\
\text { Lost }\end{array}$ \\
\hline Diagnostic Category & ICD-9-CM Codes & & & & & & & & \\
\hline -Disorders of Eye & $360-379$ & 0 & 0 & 0 & 0 & 0 & 0 & 0 & 0 \\
\hline -Dorsopathies & $720-724$ & 0 & 0 & 0 & 0 & 0 & 0 & 0 & 0 \\
\hline -Fracture - Upper Limb & 810-819 & 0 & 37 & 0 & 0 & 0 & 0 & 0 & 0 \\
\hline -Sprains \& Strains - Back & 846-847 & 0 & 0 & 0 & 0 & 0 & 0 & 0 & 0 \\
\hline -Sprains \& Strains - Other & $840-845,848$ & 29 & 12 & 0 & 0 & 48 & 8 & 0 & 0 \\
\hline -Open Wound - Head, Neck, Trunk & $870-879$ & 0 & 0 & 0 & 0 & 0 & 0 & 0 & 0 \\
\hline -Open Wound - Upper Limb & 880-887 & 0 & 0 & 0 & 0 & 0 & 0 & 0 & 0 \\
\hline -Superficial Injury & 910-919 & 0 & 0 & 0 & 0 & 0 & 2 & 0 & 0 \\
\hline -Crushing Injury & $925-929$ & 3 & 1 & 0 & 0 & 0 & 0 & 0 & 0 \\
\hline -Burns & $940-949$ & 0 & 0 & 0 & 0 & 0 & 0 & 0 & 0 \\
\hline $\begin{array}{l}\text {-Complications \& Unspecified } \\
\text { Injuries }\end{array}$ & 958-959 & 0 & 0 & 6 & 6 & 62 & 53 & 88 & 147 \\
\hline
\end{tabular}

*OSHA events with >1 ICD-9-CM code in the same diagnostic category were counted only once. Only those diagnostic categories and gender/age combinations with at least one occurrence appear in this table. 
Brookhaven National Laboratory 2010

OSHA Data

Appendix Q. Number of Occurrences in Each Accident Category by Gender and Age*

\begin{tabular}{|c|c|c|c|c|c|c|c|}
\hline & & \multicolumn{2}{|c|}{ Women } & \multicolumn{3}{|c|}{ Men } & \multirow[b]{3}{*}{ TOTAL } \\
\hline & & Age Group & \multirow[b]{2}{*}{ TOTAL } & \multicolumn{2}{|c|}{ Age Group } & \multirow[b]{2}{*}{ TOTAL } & \\
\hline & & $50+$ & & $40-49$ & $50+$ & & \\
\hline Type of Accident & E Codes & & & & & & \\
\hline Falls & E880-E888 & 2 & 2 & 3 & 2 & 5 & 7 \\
\hline Natural/Environmental Factors & E900-E909 & 1 & 1 & 0 & 0 & 0 & 1 \\
\hline Other Accidents & E916-E928 & 0 & 0 & 5 & 5 & 10 & 10 \\
\hline
\end{tabular}

*Only those accident types and gender/age combinations with at least one occurrence appear in this table. 
Brookhaven National Laboratory 2010

OSHA Data

Appendix R. Number of Workdays Lost or with Restricted Activity in Each Accident Category by Gender and Age*

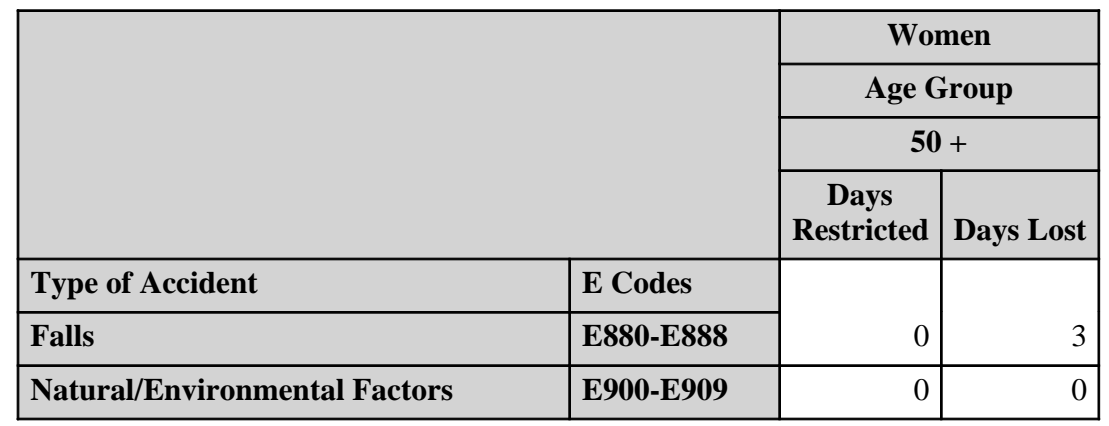

\begin{tabular}{|c|c|c|c|c|c|}
\hline & & \multicolumn{4}{|c|}{ Men } \\
\hline & & \multicolumn{4}{|c|}{ Age Group } \\
\hline & & \multicolumn{2}{|c|}{$40-49$} & \multicolumn{2}{|c|}{$50+$} \\
\hline & & $\begin{array}{c}\text { Days } \\
\text { Restricted }\end{array}$ & Days Lost & $\begin{array}{c}\text { Days } \\
\text { Restricted }\end{array}$ & Days Lost \\
\hline Type of Accident & E Codes & \multirow[b]{2}{*}{94} & \multirow[b]{2}{*}{18} & \multirow[b]{2}{*}{0} & \multirow[b]{2}{*}{123} \\
\hline Falls & E880-E888 & & & & \\
\hline Other Accidents & E916-E928 & 3 & 1 & 88 & 24 \\
\hline
\end{tabular}

*OSHA events with $>1 \mathrm{E}$ code in the same accident type were counted only once. Only those accident types and gender/age combinations with at least one occurrence appear in this table. 
Brookhaven National Laboratory 2010

OSHA Data

Appendix S. Number of Diagnoses in Each Diagnostic Category by Gender and Job Category*

\begin{tabular}{|c|c|c|c|c|}
\hline & & \multicolumn{3}{|c|}{ Women } \\
\hline & & \multicolumn{2}{|c|}{ Job Category } & \multirow[b]{2}{*}{ TOTAL } \\
\hline & & Professional & $\begin{array}{c}\text { Administrative } \\
\text { Support }\end{array}$ & \\
\hline Diagnostic Category & ICD-9-CM Code & \multirow[b]{2}{*}{3} & \multirow[b]{2}{*}{2} & \multirow[b]{2}{*}{5} \\
\hline INJURY \& POISONING & 800-999 & & & \\
\hline -Burns & 940-949 & 1 & 0 & 1 \\
\hline $\begin{array}{l}\text {-Complications \& } \\
\text { Unspecified Injuries }\end{array}$ & 958-959 & 1 & 2 & 3 \\
\hline $\begin{array}{l}\text {-Toxic } \\
\text { Effects - Non-medicinal }\end{array}$ & 980-989 & 1 & 0 & 1 \\
\hline
\end{tabular}

\begin{tabular}{|l|r|r|r|}
\hline \multirow{4}{*}{} & \multicolumn{3}{|c|}{ Women } \\
\cline { 2 - 4 } & Job Category & \\
\cline { 2 - 4 } & Professional & $\begin{array}{c}\text { Administrative } \\
\text { Support }\end{array}$ & TOTAL \\
\hline Diagnostic Category & 3 & & \\
\hline Total & & 2 & 5 \\
\hline
\end{tabular}

\begin{tabular}{|c|c|c|c|c|c|c|c|}
\hline & \multicolumn{6}{|c|}{ Men } \\
\hline & & \multicolumn{5}{|c|}{ Job Category } & \multirow[b]{2}{*}{ TOTAL } \\
\hline & & Professional & \begin{tabular}{|l|} 
Technical Support \\
\end{tabular} & Service & Security and Fire & Crafts & \\
\hline Diagnostic Category & \begin{tabular}{|l|} 
ICD-9-CM Code \\
\end{tabular} & & & & & & \\
\hline $\begin{array}{l}\text { NERVOUS SYSTEM } \\
\text { (NS) \& SENSE ORGANS }\end{array}$ & $320-389$ & 0 & 0 & 0 & 1 & 0 & 1 \\
\hline -Disorders of Eye & $360-379$ & 0 & 0 & 0 & 1 & 0 & 1 \\
\hline $\begin{array}{l}\text { MUSCULOSKELETAL } \\
\text { \& CONNECTIVE } \\
\text { TISSUE }\end{array}$ & $710-739$ & 0 & 0 & 1 & 0 & 0 & 1 \\
\hline -Dorsopathies & $720-724$ & 0 & 0 & 1 & 0 & 0 & 1 \\
\hline INJURY \& POISONING & $800-999$ & 2 & 4 & 4 & 3 & 9 & 22 \\
\hline -Fracture - Upper Limb & $810-819$ & 0 & 0 & 0 & 0 & 1 & 1 \\
\hline -Sprains \& Strains - Back & 846-847 & 0 & 1 & 0 & 0 & 0 & 1 \\
\hline $\begin{array}{l}\text {-Sprains \& Strains - } \\
\text { Other }\end{array}$ & $840-845,848$ & 0 & 1 & 0 & 1 & 0 & 2 \\
\hline $\begin{array}{l}\text {-Open Wound - Head, } \\
\text { Neck, Trunk }\end{array}$ & $870-879$ & 1 & 0 & 0 & 0 & 0 & 1 \\
\hline $\begin{array}{l}\text {-Open Wound - Upper } \\
\text { Limb }\end{array}$ & $880-887$ & 0 & 1 & 1 & 0 & 0 & 2 \\
\hline -Superficial Injury & 910-919 & 0 & 0 & 0 & 1 & 0 & 1 \\
\hline -Crushing Injury & 925-929 & 0 & 0 & 0 & 0 & 1 & 1 \\
\hline -Burns & 940-949 & 1 & 0 & 0 & 0 & 0 & 1 \\
\hline $\begin{array}{l}\text {-Complications \& } \\
\text { Unspecified Injuries }\end{array}$ & 958-959 & 0 & 1 & 3 & 1 & 7 & 12 \\
\hline
\end{tabular}

*Only those diagnostic categories and gender/job category combinations with at least one occurrence appear in this table. 
Brookhaven National Laboratory 2010

OSHA Data

Appendix S. Number of Diagnoses in Each Diagnostic Category by Gender and Job Category*

\begin{tabular}{|l|r|r|r|r|r|r|}
\hline & \multicolumn{7}{|c|}{ Men } & \\
\cline { 2 - 7 } & \multicolumn{5}{|c|}{ Job Category } \\
\cline { 2 - 7 } & Professional & Technical Support & Service & Security and Fire & Crafts & TOTAL \\
\hline Diagnostic Category & & & & & & \\
\cline { 1 - 7 } Total & 2 & 4 & 5 & 9 & 24 \\
\hline
\end{tabular}

*Only those diagnostic categories and gender/job category combinations with at least one occurrence appear in this table. 
Brookhaven National Laboratory 2010

OSHA Data

Appendix T. Number of Workdays Lost or with Restricted Activity in Each Diagnostic Category by Gender and Job Category*

\begin{tabular}{|c|c|c|c|c|c|}
\hline & & \multicolumn{4}{|c|}{ Women } \\
\hline & & \multicolumn{4}{|c|}{ Job Category } \\
\hline & & \multicolumn{2}{|c|}{ Professional } & \multicolumn{2}{|c|}{$\begin{array}{l}\text { Administrative } \\
\text { Support }\end{array}$} \\
\hline & & $\begin{array}{c}\text { Days } \\
\text { Restricted }\end{array}$ & Days Lost & \begin{tabular}{|c|} 
Days \\
Restricted
\end{tabular} & Days Lost \\
\hline Diagnostic Category & ICD-9-CM Codes & \multirow[b]{2}{*}{0} & \multirow[b]{2}{*}{0} & \multirow[b]{2}{*}{0} & \multirow[b]{2}{*}{0} \\
\hline -Burns & $940-949$ & & & & \\
\hline $\begin{array}{l}\text {-Complications \& Unspecified } \\
\text { Injuries }\end{array}$ & 958-959 & 0 & 3 & 0 & 0 \\
\hline -Toxic Effects - Non-medicinal & $980-989$ & 0 & 0 & 0 & 0 \\
\hline
\end{tabular}

*OSHA events with >1 ICD-9-CM code in the same diagnostic category were counted only once. Only those diagnostic categories and gender/job category combinations with at least one occurrence appear in this table. 
Brookhaven National Laboratory 2010

OSHA Data

Appendix T. Number of Workdays Lost or with Restricted Activity in Each Diagnostic Category by Gender and Job Category*

\begin{tabular}{|c|c|c|c|c|c|c|c|}
\hline & & \multicolumn{6}{|c|}{ Men } \\
\hline & & \multicolumn{6}{|c|}{ Job Category } \\
\hline & & \multicolumn{2}{|c|}{ Professional } & \multicolumn{2}{|c|}{ Technical Support } & \multicolumn{2}{|c|}{ Service } \\
\hline & & $\begin{array}{c}\text { Days } \\
\text { Restricted }\end{array}$ & Days Lost & $\begin{array}{c}\text { Days } \\
\text { Restricted }\end{array}$ & Days Lost & $\begin{array}{c}\text { Days } \\
\text { Restricted }\end{array}$ & Days Lost \\
\hline Diagnostic Category & ICD-9-CM Codes & \multirow[b]{2}{*}{0} & \multirow[b]{2}{*}{0} & \multirow[b]{2}{*}{0} & \multirow[b]{2}{*}{0} & \multirow[b]{2}{*}{0} & \multirow[b]{2}{*}{0} \\
\hline -Disorders of Eye & $360-379$ & & & & & & \\
\hline -Dorsopathies & $720-724$ & 0 & 0 & 0 & 0 & 0 & 0 \\
\hline -Fracture - Upper Limb & 810-819 & 0 & 0 & 0 & 0 & 0 & 0 \\
\hline -Sprains \& Strains - Back & 846-847 & 0 & 0 & 0 & 0 & 0 & 0 \\
\hline -Sprains \& Strains - Other & $840-845,848$ & 0 & 0 & 29 & 12 & 0 & 0 \\
\hline -Open Wound - Head, Neck, Trunk & $870-879$ & 0 & 0 & 0 & 0 & 0 & 0 \\
\hline -Open Wound - Upper Limb & $880-887$ & 0 & 0 & 0 & 0 & 0 & 0 \\
\hline -Superficial Injury & 910-919 & 0 & 0 & 0 & 0 & 0 & 0 \\
\hline -Crushing Injury & $925-929$ & 0 & 0 & 0 & 0 & 0 & 0 \\
\hline -Burns & $940-949$ & 0 & 0 & 0 & 0 & 0 & 0 \\
\hline $\begin{array}{l}\text {-Complications \& Unspecified } \\
\text { Injuries }\end{array}$ & \begin{tabular}{|c|}
$958-959$ \\
\end{tabular} & 0 & 0 & 0 & 0 & 31 & 134 \\
\hline
\end{tabular}

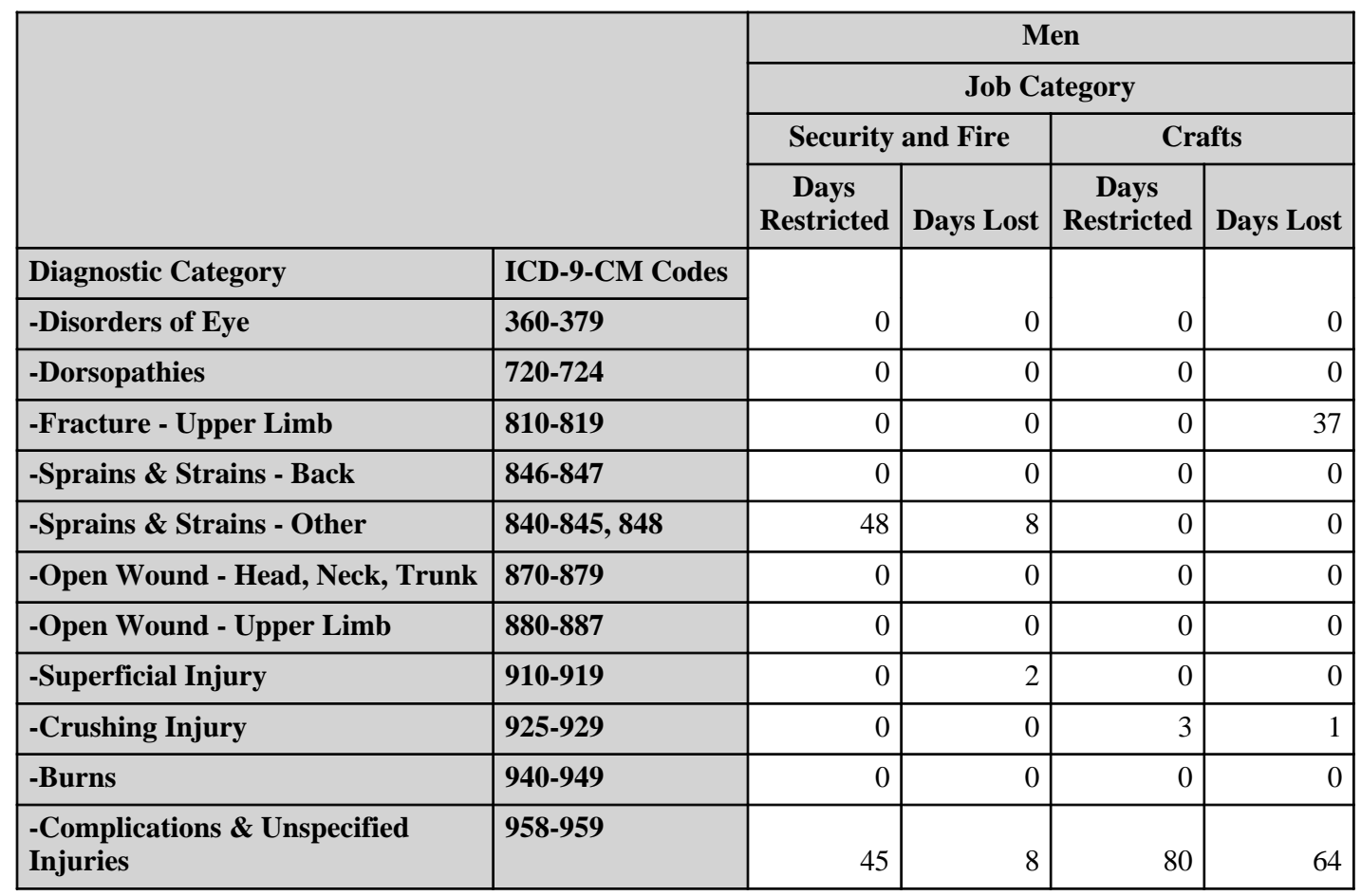

*OSHA events with >1 ICD-9-CM code in the same diagnostic category were counted only once. Only those diagnostic categories and gender/job category combinations with at least one occurrence appear in this table. 
Brookhaven National Laboratory 2010

OSHA Data

Appendix U. Number of Occurrences in Each Accident Category by Gender and Job Category*

\begin{tabular}{|c|c|c|c|c|}
\hline & & \multicolumn{3}{|c|}{ Women } \\
\hline & & \multicolumn{2}{|c|}{ Job Category } & \multirow[b]{2}{*}{ TOTAL } \\
\hline & & Professional & $\begin{array}{c}\text { Administrative } \\
\text { Support }\end{array}$ & \\
\hline Type of Accident & E CODES & \multirow[b]{2}{*}{1} & \multirow[b]{2}{*}{1} & \multirow[b]{2}{*}{2} \\
\hline Falls & E880-E888 & & & \\
\hline Natural/Environmental Factors & E900-E909 & 1 & 0 & 1 \\
\hline
\end{tabular}

\begin{tabular}{|c|c|c|c|c|c|c|c|}
\hline & & \multicolumn{6}{|c|}{ Men } \\
\hline & & \multicolumn{5}{|c|}{ Job Category } & \multirow[b]{2}{*}{ TOTAL } \\
\hline & & Professional & $\begin{array}{c}\text { Technical } \\
\text { Support }\end{array}$ & Service & \begin{tabular}{|c|}
$\begin{array}{c}\text { Security and } \\
\text { Fire }\end{array}$ \\
\end{tabular} & Crafts & \\
\hline Type of Accident & E CODES & \multirow[b]{2}{*}{1} & \multirow[b]{2}{*}{0} & \multirow[b]{2}{*}{1} & \multirow[b]{2}{*}{1} & \multirow[b]{2}{*}{2} & \multirow[b]{2}{*}{5} \\
\hline Falls & E880-E888 & & & & & & \\
\hline Other Accidents & E916-E928 & 1 & 2 & 3 & 1 & 3 & 10 \\
\hline
\end{tabular}

*Only those accident types and gender/job category combinations with at least one occurrence appear in this table. 
Brookhaven National Laboratory 2010

OSHA Data

Appendix V. Number of Workdays Lost or with Restricted Activity in Each Accident Category by Gender and Job Category*

\begin{tabular}{|c|c|c|c|c|c|}
\hline & & \multicolumn{4}{|c|}{ Women } \\
\hline & & \multicolumn{4}{|c|}{ Job Category } \\
\hline & & \multicolumn{2}{|c|}{ Professional } & \multicolumn{2}{|c|}{$\begin{array}{l}\text { Administrative } \\
\text { Support }\end{array}$} \\
\hline & & $\begin{array}{c}\text { Days } \\
\text { Restricted }\end{array}$ & Days Lost & $\begin{array}{c}\text { Days } \\
\text { Restricted }\end{array}$ & Days Lost \\
\hline Type of Accident & E Codes & \multirow[b]{2}{*}{0} & \multirow[b]{2}{*}{3} & \multirow[b]{2}{*}{0} & \multirow[b]{2}{*}{0} \\
\hline Falls & E880-E888 & & & & \\
\hline Natural/Environmental Factors & E900-E909 & 0 & 0 & 0 & 0 \\
\hline
\end{tabular}

\begin{tabular}{|c|c|c|c|c|c|c|c|}
\hline & & \multicolumn{6}{|c|}{ Men } \\
\hline & & \multicolumn{6}{|c|}{ Job Category } \\
\hline & & \multicolumn{2}{|c|}{ Professional } & \multicolumn{2}{|c|}{ Technical Support } & \multicolumn{2}{|c|}{ Service } \\
\hline & & $\begin{array}{c}\text { Days } \\
\text { Restricted }\end{array}$ & Days Lost & $\begin{array}{c}\text { Days } \\
\text { Restricted }\end{array}$ & Days Lost & $\begin{array}{c}\text { Days } \\
\text { Restricted }\end{array}$ & Days Lost \\
\hline Type of Accident & E Codes & \multirow[b]{2}{*}{0} & \multirow[b]{2}{*}{0} & \multirow[b]{2}{*}{0} & \multirow[b]{2}{*}{0} & \multirow[b]{2}{*}{0} & \multirow[b]{2}{*}{123} \\
\hline Falls & \begin{tabular}{|l|} 
E880-E888 \\
\end{tabular} & & & & & & \\
\hline Other Accidents & E916-E928 & 0 & 0 & 0 & 0 & 31 & 11 \\
\hline
\end{tabular}

\begin{tabular}{|c|c|c|c|c|c|}
\hline & & \multicolumn{4}{|c|}{ Men } \\
\hline & & \multicolumn{4}{|c|}{ Job Category } \\
\hline & & \multicolumn{2}{|c|}{ Security and Fire } & \multicolumn{2}{|c|}{ Crafts } \\
\hline & & $\begin{array}{c}\text { Days } \\
\text { Restricted }\end{array}$ & Days Lost & $\begin{array}{c}\text { Days } \\
\text { Restricted }\end{array}$ & Days Lost \\
\hline Type of Accident & E Codes & \multirow[b]{2}{*}{48} & \multirow[b]{2}{*}{8} & \multirow[b]{2}{*}{46} & \multirow[b]{2}{*}{10} \\
\hline Falls & E880-E888 & & & & \\
\hline Other Accidents & E916-E928 & 45 & 8 & 15 & 6 \\
\hline
\end{tabular}

*OSHA events with >1 E code in the same accident type were counted only once. Only those accident types and gender/job category combinations with at least one occurrence appear in this table. 
Brookhaven National Laboratory 2010

OSHA Data

Appendix W. Age-Adjusted OSHA Illness and Injury Rates by Diagnostic Category*

Part 1. Men

\begin{tabular}{|c|c|c|c|c|c|}
\hline & & $\begin{array}{l}\text { Number of } \\
\text { Diagnoses }\end{array}$ & $\begin{array}{c}\text { Age-Adjusted } \\
\text { Rate per } 1,000 * *\end{array}$ & $\begin{array}{c}\text { Lower } 95 \% \\
\text { Confidence } \\
\text { Limit per 1,000 }\end{array}$ & $\begin{array}{c}\text { Upper } 95 \% \\
\text { Confidence } \\
\text { Limit per } 1,000\end{array}$ \\
\hline Diagnostic Category & ICD-9-CM Code & \multirow[b]{2}{*}{1} & \multirow[b]{2}{*}{0.4} & \multirow[b]{2}{*}{0.1} & \multirow[b]{2}{*}{2.7} \\
\hline NERVOUS SYSTEM (NS) \& SENSE ORGANS & 320-389 & & & & \\
\hline -Disorders of Eye & $360-379$ & 1 & 0.4 & 0.1 & 2.7 \\
\hline $\begin{array}{l}\text { MUSCULOSKELETAL \& CONNECTIVE } \\
\text { TISSUE }\end{array}$ & $710-739$ & 1 & 0.3 & 0.0 & 1.9 \\
\hline -Dorsopathies & $720-724$ & 1 & 0.3 & 0.0 & 1.9 \\
\hline INJURY \& POISONING & $800-999$ & 22 & 11.2 & 6.4 & 19.7 \\
\hline -Fracture - Upper Limb & $810-819$ & 1 & 1.6 & 0.2 & 11.6 \\
\hline -Sprains \& Strains - Back & 846-847 & 1 & 0.4 & 0.1 & 2.7 \\
\hline -Sprains \& Strains - Other & $840-845,848$ & 2 & 2.0 & 0.4 & 10.3 \\
\hline -Open Wound - Head, Neck, Trunk & $870-879$ & 1 & 0.3 & 0.0 & 1.9 \\
\hline -Open Wound - Upper Limb & $880-887$ & 2 & 0.8 & 0.2 & 3.0 \\
\hline -Superficial Injury & $910-919$ & 1 & 0.4 & 0.1 & 2.7 \\
\hline -Burns & $940-949$ & 1 & 0.2 & 0.0 & 1.6 \\
\hline -Complications of Surgical/Medical Care & 996-999 & 13 & 5.6 & 2.7 & 11.4 \\
\hline Total & & 24 & 11.9 & 6.9 & 20.3 \\
\hline
\end{tabular}

* Only those diagnostic categories with at least one occurrence appear in this table.

**Standardized to age distribution of 2000 U.S. population. 
Brookhaven National Laboratory 2010

OSHA Data

Appendix W. Age-Adjusted OSHA Illness and Injury Rates by Diagnostic Category*

Part 2. Women

\begin{tabular}{|c|c|c|c|c|c|}
\hline & & $\begin{array}{l}\text { Number of } \\
\text { Diagnoses }\end{array}$ & $\begin{array}{c}\text { Age-Adjusted } \\
\text { Rate per } 1,000 * *\end{array}$ & $\begin{array}{c}\text { Lower } 95 \% \\
\text { Confidence } \\
\text { Limit per } 1,000\end{array}$ & $\begin{array}{c}\text { Upper } 95 \% \\
\text { Confidence } \\
\text { Limit per 1,000 }\end{array}$ \\
\hline Diagnostic Category & ICD-9-CM Code & \multirow[b]{2}{*}{5} & \multirow[b]{2}{*}{5.3} & \multirow[b]{2}{*}{2.0} & \multirow[b]{2}{*}{14.2} \\
\hline INJURY \& POISONING & $800-999$ & & & & \\
\hline -Burns & $940-949$ & 1 & 1.7 & 0.2 & 12.3 \\
\hline -Toxic Effects - Non-medicinal & 980-989 & 1 & 0.6 & 0.1 & 4.3 \\
\hline -Complications of Surgical/Medical Care & 996-999 & 3 & 3.0 & 0.8 & 10.7 \\
\hline Total & & 5 & 5.3 & 2.0 & 14.2 \\
\hline
\end{tabular}

*Only those diagnostic categories with at least one occurrence appear in this table.

**Standardized to age distribution of 2000 U.S. population. 
Brookhaven National Laboratory 2010

OSHA Data

Appendix W. Age-Adjusted OSHA Illness and Injury Rates by Diagnostic Category*

Part 3. Men and Women

\begin{tabular}{|c|c|c|c|c|c|}
\hline & & $\begin{array}{l}\text { Number of } \\
\text { Diagnoses }\end{array}$ & $\begin{array}{c}\text { Age-Adjusted } \\
\text { Rate per } 1,000 * *\end{array}$ & $\begin{array}{c}\text { Lower } 95 \% \\
\text { Confidence } \\
\text { Limit per 1,000 }\end{array}$ & $\begin{array}{c}\text { Upper } 95 \% \\
\text { Confidence } \\
\text { Limit per 1,000 }\end{array}$ \\
\hline Diagnostic Category & ICD-9-CM Code & \multirow[b]{2}{*}{1} & \multirow[b]{2}{*}{0.3} & \multirow[b]{2}{*}{0.0} & \multirow[b]{2}{*}{1.9} \\
\hline NERVOUS SYSTEM (NS) \& SENSE ORGANS & 320-389 & & & & \\
\hline -Disorders of Eye & $360-379$ & 1 & 0.3 & 0.0 & 1.9 \\
\hline $\begin{array}{l}\text { MUSCULOSKELETAL \& CONNECTIVE } \\
\text { TISSUE }\end{array}$ & 710-739 & 1 & 0.2 & 0.0 & 1.5 \\
\hline -Dorsopathies & $720-724$ & 1 & 0.2 & 0.0 & 1.5 \\
\hline INJURY \& POISONING & $800-999$ & 27 & 9.3 & 5.7 & 15.2 \\
\hline -Fracture - Upper Limb & 810-819 & 1 & 1.1 & 0.2 & 8.0 \\
\hline -Sprains \& Strains - Back & 846-847 & 1 & 0.3 & 0.0 & 1.9 \\
\hline -Sprains \& Strains - Other & $840-845,848$ & 2 & 1.4 & 0.3 & 7.1 \\
\hline -Open Wound - Head, Neck, Trunk & 870-879 & 1 & 0.2 & 0.0 & 1.5 \\
\hline -Open Wound - Upper Limb & 880-887 & 2 & 0.5 & 0.1 & 2.2 \\
\hline -Superficial Injury & 910-919 & 1 & 0.3 & 0.0 & 1.9 \\
\hline -Burns & 940-949 & 2 & 0.6 & 0.1 & 2.8 \\
\hline -Toxic Effects - Non-medicinal & 980-989 & 1 & 0.2 & 0.0 & 1.2 \\
\hline -Complications of Surgical/Medical Care & 996-999 & 16 & 4.8 & 2.6 & 8.9 \\
\hline Total & & 29 & 9.8 & 6.1 & 15.7 \\
\hline
\end{tabular}

* Only those diagnostic categories with at least one occurrence appear in this table.

**Standardized to age distribution of 2000 U.S. population. 